\title{
Longitudinal Coupling Impedance of a Hole In the Accelerator Beam Pipe
}

\author{
Yong-Chul Chae \\ Advanced Photon Source Project \\ Argonne National Laboratory \\ 9700 South Cass Avenue, Argonne, IL 60439
}

December 1993

DISCLAIMER

This report was prepared as an account of work sponsored by an agency of the United States Government. Neither the United States Government nor any agency thereof, nor any of their employees, makes any warranty, express or implied, or assumes any legal liability or responsibility for the accuracy, completeness, or usefulness of any information, apparatus, product, or process disclosed, or represents that its use would not infringe privately owned rights. Reference herein to any specific commercial product, process, or service by trade name, trademark, manufacturer, or otherwise does not necessarily constitute or imply its endorsement, recommendation, or favoring by the United States Government or any agency thereof. The views and opinions of authors expressed herein do not necessarily state or reflect those of the United States Government or any agency thereof. 


\section{Abstract}

In the design of modern accelerators, an accurate estimate of coupling impedance is very important. The sources which give rise to coupling impedance are the geometric discontinuities in the accelerator beam pipe. In various discontinuities such as RF cavities, bellows, and collimators, the coupling impedance of the holes has not been well understood. Although coupling impedance can be obtained in general from the Fourier transform of the corresponding wake potential which may be obtained numerically, this is time consuming and requires a large amount of computer storage when applied to a small dimension of a discontinuity in a typical beam pipe, often imposing a fundamental limitation of the numerical approach. It is especially true for the holes since the typical size of a hole is small compared with the dimension of a typical beam pipe. More fundamentally, however, numerical calculation does not have the predictive power because of limited understanding of how the coupling impedance of a hole should behave over a wide frequency range. This question was studied by developing a theoretical analysis based on a variational method.

An analytical formula for the coupling impedance of a hole is developed in this work using a variational method. The result gives good qualitative agreements with the coupling impedances evaluated numerically from the Fourier transform of the wake potential which is obtained from the computer code MAFIA-T3. We show that the coupling impedance of a hole behaves quite similar to the impedance of an RLC-resonator circuit. Important parameters used to describe such a resonator circuit are the resonant frequency and bandwidth. These parameters can be easily determined from the formula presented in this work. We provide a theoretical insight on how to parameterize properly the numerical impedance of a hole when data exhibit 


\section{DISCLAIMER}

Portions of this document may be illegible in electronic image products. Images are produced from the best available original document. 
complicated dependence on frequency. This is possible because we can show that the parameters are a function of the dimensionless quantity $k d$ alone, with $k$ the free-space wave number and $d$ the radius of hole.

In summary, we will develop an analytical method for the hole-coupling problem valid for a wide range of frequencies. 


\section{Contents}

1 Introduction $\quad 1$

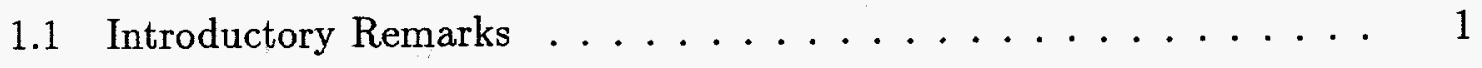

1.2 Definition of Longitudinal Wake Potential and Coupling Impedance . 2

1.3 Aperture Problem and Gluckstern's Formalism . . . . . . . . . . 6

1.4 Goal and Scope of the Work ................ . . 8

2 Vector Diffraction Theory $\quad 10$

2.1 Introduction . . . . . . . . . . . . . . 10

2.2 Field Equivalence Principle. . . . . . . . . . . . . 12

2.3 Free-Space Dyadic Green's Function . . . . . . . . . . . 15

2.4 Dyadic Green's Function and Field Equivalence Principle . . . . . . . 20

2.5 Reaction Concept and Variational Formalism . . . . . . . . . . 23

2.6 Application to Aperture Problem in an Infinite Screen . . . . . . 25

3 Longitudinal Impedance of a Hole in an Infinite Screen 35

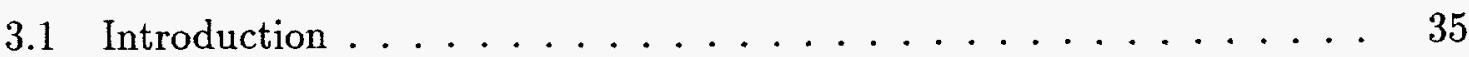

3.2 Low Frequency Solution . . . . . . . . . . . . . 36

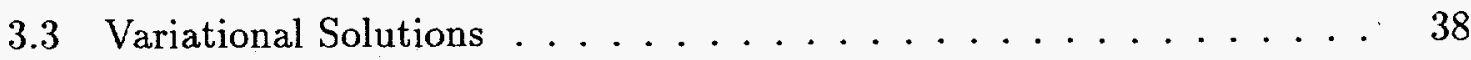

3.3.1 Derivation of Impedance Functional . . . . . . . . . . . 39 
3.3.2 The First Variational Solution . . . . . . . . . . . 41

3.3.3 The Second Variational Solution ............. 56

4 Longitudinal Impedance of a Hole in the Accelerator Beam Pipe 70

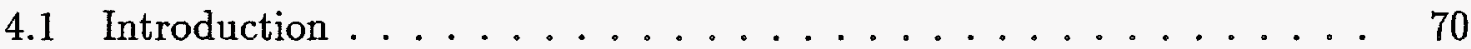

4.2 Low Frequency Solution . . . . . . . . . . . . 71

4.3 Variational Solution ....................... 74

4.4 Comparison with Numerical Results . . . . . . . . . . . . 82

5 Summary and Conclusion $\quad 96$

$\begin{array}{ll}\text { Bibliography } & 98\end{array}$ 


\section{List of Figures}

1.1 Leading (driving) and trailing (test) charges in a pill-box cavity with axial symmetry. . . . . . . . . . . . . . . 3

1.2 (a) A charge traveling in the smooth beam pipe, (b) a charge traveling

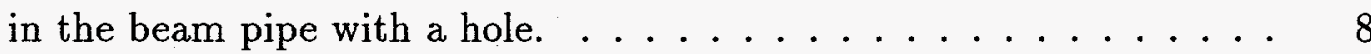

2.1 Illustration of Love's field equivalence principle. (a) Original problem; (b) equivalent to $(a) \ldots \ldots \ldots \ldots \ldots \ldots \ldots \ldots$

2.2 Illustration of Schelkunoff's field equivalence principle. (a) magnetic current over electric conductor; (b) electric current over magnetic conductor. . . . . . . . . . . . . . . . 14

2.3 Diffracting aperture in a plane screen. . . . . . . . . . . 26

2.4 (a) Original problem; (b) and (c) Equivalent problem to (a) for the region $z \geq 0 \ldots \ldots \ldots \ldots \ldots \ldots \ldots$

2.5 (a) Original problem; (b) and (c) Equivalent problem to (a) for the region $z \leq 0 \ldots \ldots \ldots \ldots \ldots \ldots \ldots \ldots \ldots \ldots$

3.1 Infinite flat screen with a hole. . . . . . . . . . . 36

3.2 Form factor as functions of $k d \ldots \ldots \ldots \ldots \ldots \ldots$

3.3 Impedance Functional $\mathcal{Z}^{(1)}(k d)$ for various $d / b$ values. . . . . . . 54

3.4 Impedance Functional $\mathcal{Z}^{(2)}(k d)$ for various $d / b$ values. $\ldots \ldots \ldots \quad 55$ 
3.5 Longitudinal coupling impedance, $Z_{H}^{(1)}(k d)$ and $Z_{E}^{(1)}(k d)$, for $d / b=0.5 . \quad 67$

3.6 Longitudinal coupling impedance $Z^{(2)}(k d)$ for various $d / b$ values. . . 68

3.7 Longitudinal coupling impedance $Z^{(3)}(k d)$ for various $d / b$ values. . . . 69

4.1 Rectangular wave guide coupled by a hole as a model to the accelerator beam pipe with a hole. ................. 71

4.2 Hole in waveguide, its equivalent magnetic surface current and its images in infinite space. . . . . . . . . . . . . . 75

4.3 Geometry used in MAFIA-T3 simulation. . . . . . . . . . . 86

4.4 Bunch potential from MAFIA-T3 simulation for the hole with radius

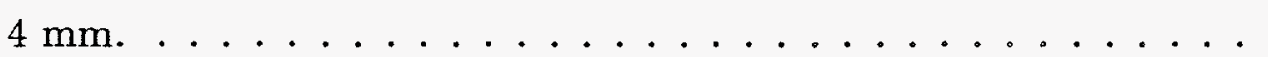

4.5 Bunch potential from MAFIA-T3 simulation for the hole with radius $1 \mathrm{~mm} \ldots \ldots \ldots \ldots \ldots \ldots$

4.6 Fourier transform of bunch potential from MAFIA-T3 simulation for the hole with radius $4 \mathrm{~mm}$. . . . . . . . . . . . 89

4.7 Coupling impedance of a point charge for the hole with radius $4 \mathrm{~mm}$. 90

4.8 Impedance calculation using the symmetry. Solid line from full geometry, dashed line from half geometry with $\mathbf{E}_{\|}=0$ and dot-dashed line from half geometry with $\mathbf{H}_{\|}=0 . \ldots \ldots . \ldots 91$

4.9 Coupling impedance of the hole with radius $1 \mathrm{~mm}$. Solid line from the variational calculation and dashed line from MAFIA-T3 simulation. . 92

4.10 Coupling impedance of the hole with radius $1 \mathrm{~mm}$ including thickness effect. Solid line from the variational calculation and dashed line from MAFIA-T3 simulation. . . . . . . . . . . . . . 
4.11 Coupling impedance of the hole with radius $2 \mathrm{~mm}$. Solid line from the variational calculation and dashed line from MAFIA-T3 simulation. . 94

4.12 Coupling impedance of the hole with radius $4 \mathrm{~mm}$. Solid line from the variational calculation and dashed line from MAFIA-T3 simulation. . 


\section{List of Tables}

4.1 Geometric Series ..................... 81 


\section{Chapter 1}

\section{Introduction}

\subsection{Introductory Remarks}

In a circular accelerator, a charged particle gains energy from the electromagnetic field in RF cavities. In electron storage rings, RF power should compensate the synchrotron radiation loss experienced by the charged particle. While an accelerator is designed such that particles execute a stable longitudinal oscillation in the potential well provided by the RF system, they should be stable in the transverse directions as well. For transverse motion, the interaction is between the charged particle and the external magnetic field which provides the transverse focusing force. Since both RF fields and magnetic fields are supplied by the system external to particles, the motion in such a field does not depend on the intensity of the beam. Hence we may call this type of motion single particle dynamics in contrast to a collective motion which is affected by the beam intensity also.

When an accelerator beam pipe is smooth everywhere, single particle dynamics alone is sufficient in understanding the behavior of the beam inside an accelerator. In general, however, an accelerator beam pipe is not smooth but discontinuous in its dimension. An obvious discontinuity in a beam pipe is the RF cavities. Other 
examples may include bellows, collimators, and pumping holes. Such unavoidable discontinuities in an accelerator beam pipe provide rich sources of interaction between the beam and the surroundings in such a way that, when the beam traverses a discontinuity, say, an RF cavity, the source field associated with the beam is scattered leaving the wake field behind the beam. The wake field then acts on the following bunch of particles or acts on the original beam itself. This self-sustained mechanism is continued either leading to a stable or to an unstable situation. Since the interaction depends on the intensity of beam, the dynamics of the system is often dominated by the so-called "collective effects."

In modern accelerators or storage rings, the maximum achievable current is limited by the collective effects. In the equation of motion which characterizes the collective effects, the concept of wake potential and its frequency domain counterpart, coupling impedance, have been introduced, providing a coherent force in the equation of motion. Thus, in designing high-intensity accelerators and storage rings, one tries to minimize the collective effects by reducing the wake potential or the coupling impedance, and this is the subject of the present work.

\subsection{Definition of Longitudinal Wake Potential and Coupling Impedance}

In this section we introduce the definition of longitudinal wake potential and coupling impedance. Even though mathematically rigorous theorems can be found in the standard literature [7], we emphasize the heuristic concept evolved over many years in accelerator physics. Once the concept of longitudinal wake potential becomes clear, extension to the transverse wake potential is straightforward. 


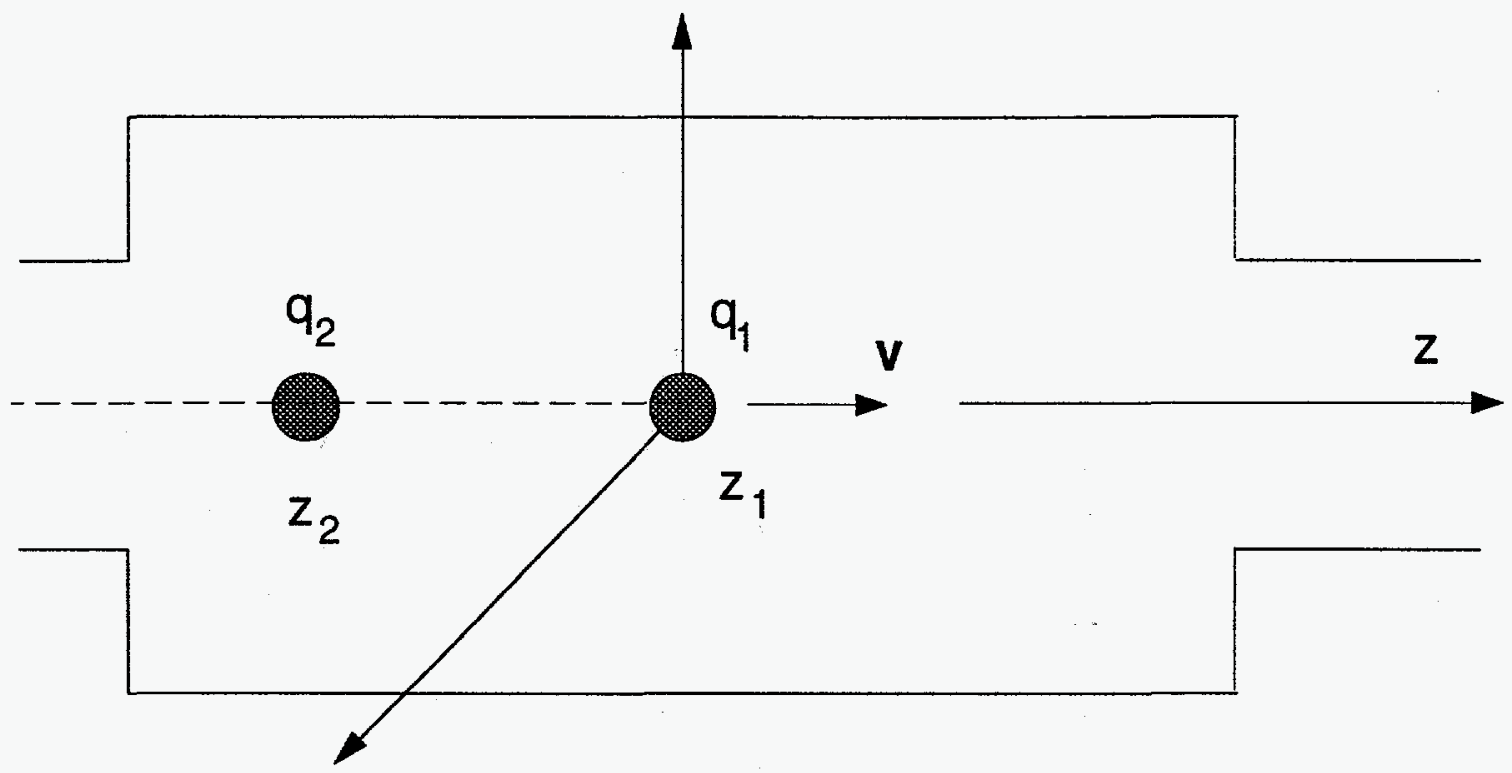

Figure 1.1: Leading (driving) and trailing (test) charges in a pill-box cavity with axial symmetry.

Let us consider two charges, $q_{1}$ and $q_{2}$, traveling with constant velocity on the axis of an axially symmetric structure. At time $t=0$, the position of leading charge is defined as $z_{1}=0$. At time $t$, leading and trailing charges have coordinates $z_{1}(t)=v t$ and $z_{2}(t)=v(t-\tau)$, where $\tau$ is the time delay of the trailing charge (see Fig. 1.1).

Since the wake field excited by $q_{1}$ exerts the Lorentz force on the trailing charge, the trailing charge experiences the energy change by an amount

$$
\Delta U=-q_{2} \int_{-\infty}^{\infty} E_{z}\left(z, t=\frac{z}{v}+\tau\right) d z
$$

where the electric field is computed at a later time and the unit is [Volt Coulomb].

We define the wake potential $W(\tau)$ as the energy lost by the trailing charge per unit of both charges $q_{1}$ and $q_{2}$ which can be expressed in the form

$$
W(\tau)=\frac{\Delta U}{q_{1} q_{2}}=-\frac{1}{q_{1}} \int_{-\infty}^{\infty} E_{z}\left(z, t=\frac{z}{v}+\tau\right) d z
$$


with the unit of [Volt/Coulomb].

Once the response to a point driving charge $q_{1}$ is calculated, the wake potential $W(\tau)$ can be used as Green's function to compute the potential in and behind an arbitrary charge distribution by means of the superposition principle. If the charge $q_{1}$ is continuously distributed according to the time distribution function $I(\tau)$ such that

$$
q_{1}=\int_{-\infty}^{\infty} I(\tau) d \tau
$$

the trailing charge experiences the effect due to a bunch distribution

$$
V(\tau)=\int_{-\infty}^{\infty} W\left(\tau-\tau^{\prime}\right) I\left(\tau^{\prime}\right) d \tau^{\prime}
$$

with the unit of [Volt]. The potential in Eq. (1.4) is sometimes called bunch potential. Since bunch potential depends on the distribution of charges, wake potential is a more fundamental quantity as it depends only on the structure surrounding a charge.

If we take the Fourier transform of Eq. (1.4), we have a response relation in the frequency domain such that

$$
Z(\omega)=W(\omega)=\frac{V(\omega)}{I(\omega)}
$$

where $W(\omega)$, a Fourier transform of wake potential, is defined as

$$
W(\omega)=\int_{-\infty}^{\infty} W(\tau) e^{-j \omega \tau} d \tau
$$

Since the quantity $Z(\omega)$ is the ratio of voltage and current, it will be called impedance or coupling impedance with $[\mathrm{Ohm}]$ as its unit.

For the calculation of bunch potential, general-purpose computer codes exist: TBCI [31] for axially symmetric geometries and MAFIA-T3 [17] for three-dimensional 
geometries. Although direct numerical calculation of wake potential is impossible because of the finite size of the mesh used in these codes, we can obtain the impedance via Eq. (1.5) from the discrete bunch potential. For the analytic calculation, however, it would be much easier to work directly in the frequency domain, noting that

$$
Z(k)=-\frac{1}{I_{0}} \int_{-\infty}^{\infty} E_{z}(z, k) e^{j k z} d z,
$$

where $k=\omega / v$ is the wave number, $I_{0}=q_{1} v$ is the $\mathrm{dc}$ current and the time dependence is assumed to be $\exp (j \omega t)$. This definition requires the field everywhere along the beam axis or along the gap if the structure is a cavity type. It should be noted also that the field integral in Eq. (1.7) is the effective voltage, including the transit time factor, experienced by the charge traversing RF cavity.

Since the drive current in the frequency domain has a sinusoidal dependence on $z$ of the form

$$
\mathbf{J}(x, y, z ; k)=I_{0} \delta(x) \delta(y) e^{-j k z} \mathbf{e}_{\mathbf{z}}
$$

we can express Eq. (1.7) as

$$
Z(k)=-\frac{1}{\left|I_{0}\right|^{2}} \int_{-\infty}^{\infty} \mathbf{E} \cdot \mathbf{J}^{*} d V
$$

In this form, coupling impedance used in the accelerator physics is equal to the input impedance of the source which excites the waveguide or cavity.

It will be shown in the next section that Eq. (1.9) is more convenient for a certain geometry, especially for a hole problem. 


\subsection{Aperture Problem and Gluckstern's Formal- ism}

Maintaining a high vacuum in a storage ring is essential for a useful beam lifetime which is typically many hours. Since the residual gas must be pumped out of the beam pipe, pumping holes are distributed around the ring for the passage of residual gas. Although the arrangement of pumping holes is different in different accelerators, the impedance of even a single hole is not a well-known quantity.

Numerical calculation using computer codes for the wake potential is possible but it will be time consuming since

1. the geometry describing a beam pipe with holes is three dimensional,

2. a large amount of computer storage will be required because the computation will need a fine mesh in order to resolve the 1 or $2 \mathrm{~mm}$ thickness of the hole-plate,

3. it is always difficult to obtain the impedance in the high frequency range because errors in the Fourier transformation are amplified in the convolution defined in $\mathrm{Eq}(1.5)$.

Finding an analytic expression for the impedance of a hole in a broad frequency range is therefore highly desirable in the design of high intensity storage rings.

Among many different ways of calculating impedance, Gluckstern's formalism $[10,11,12]$ is particularly useful, and is introduced in this section. Let us consider two axially symmetric beam pipes, one without a hole and the other with a hole shown in Fig. 1.2. If we denote the field in the smooth beam pipe as $\mathbf{E}_{1}, \mathbf{H}_{1}$, which is the source field, and the field in the beam pipe with a hole as $\mathbf{E}_{2}, \mathbf{H}_{2}$, which is the 
source field plus scattered field due to a hole, they satisfy Maxwell's equations in the form

$$
\nabla \times \mathbf{E}_{1,2}=-j \omega \mu \mathbf{H}_{1,2} \quad, \nabla \times \mathbf{H}_{1,2}=j \omega \epsilon \mathbf{H}_{1,2}+\mathbf{J},
$$

where $\mathbf{J}=I_{0} \delta(x) \delta(y) e^{-j k z} \mathbf{e}_{z}$ is the complex current density and $k=\omega \sqrt{\epsilon_{0} \mu_{0}}$ is the free-space wave number. Assuming that the velocity of traveling charge is ultrarelativistic ( $\beta=v / c \simeq 1, \gamma=1 / \sqrt{1-\beta^{2}} \gg 1$, which is assumed throughout), and using a well-known result of the impedance due to the source field

$$
Z_{1}(k)=- \text { constant } \times \frac{j}{\gamma^{2}}
$$

we can construct

$$
\left|I_{0}\right|^{2}\left[Z_{2}(k)+Z_{1}^{*}(k)\right]=\left|I_{0}\right|^{2}\left[Z_{2}(k)-Z_{1}(k)\right]=-\int\left[\mathbf{E}_{2} \cdot \mathbf{J}^{*}+\mathbf{E}_{1}^{*} \cdot \mathbf{J}\right] d V
$$

Noting the identity $\nabla \cdot\left(\mathbf{E}_{1}^{*} \times \mathbf{H}_{2}+\mathbf{E}_{2} \times \mathbf{H}_{1}^{*}\right)=-\left(\mathbf{E}_{2} \cdot \mathbf{J}^{*}+\mathbf{E}_{1}^{*} \cdot \mathbf{J}\right)$, we convert the volume integral into the surface integral, leading to

$$
\left|I_{0}\right|^{2} Z_{2}(k)=\int \mathbf{n} \cdot\left[\mathbf{E}_{2} \times \mathbf{H}_{1}^{*}+\mathbf{E}_{1}^{*} \times \mathbf{H}_{2}\right] d S,
$$

where we used the fact that $Z_{1}(k)$ vanishes in the ultrarelativistic limit. If we choose $S$ to be the inside surface of the beam pipe, $\mathbf{n} \cdot \mathbf{E}_{1}^{*} \times \mathbf{H}_{2}=0$, we have

$$
\left|I_{0}\right|^{2} Z_{2}(k)=\int_{\text {hole }}\left(\mathbf{n} \times \mathbf{E}_{2}\right) \cdot \mathbf{H}_{1}^{*}
$$

where $\mathbf{n} \times \mathbf{E}_{2}$ is the tangential field inside the hole, a quantity unknown until we solve the problem completely.

Determining the field inside hole has been an important subject of investigation for a long time. Several hundred papers have been published on this subject, and 


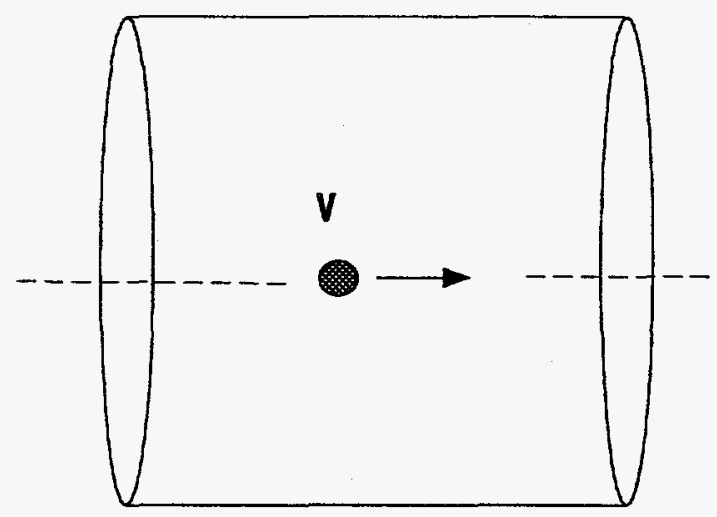

(a)

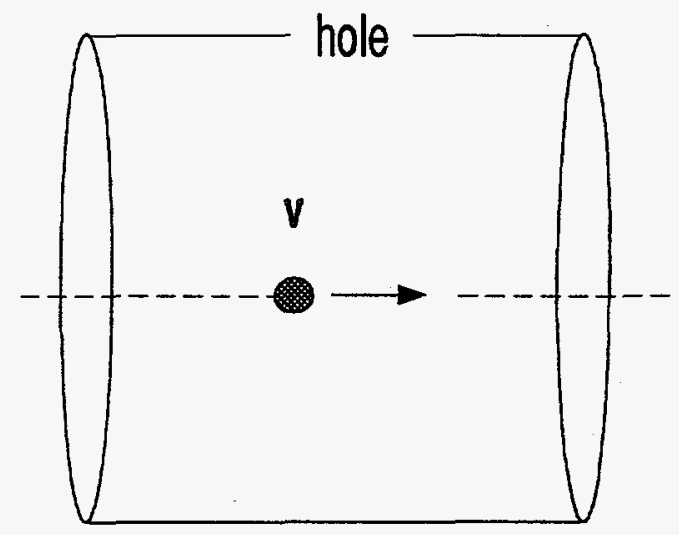

(b)

Figure 1.2: (a) A charge traveling in the smooth beam pipe, (b) a charge traveling in the beam pipe with a hole.

an excellent review paper was written by Bouwkamp in 1954 [4]. Among the large amount of literature available, Schelkunoff's Field Equivalence Theorems [26], Levine and Schwinger's variational formalism using dyadic Green's function [21], and Rumsey's Reaction Concept [23] are particularly useful for our problem. These works are summarized in Chapter 2.

\subsection{Goal and Scope of the Work}

Obtaining an analytical estimate of longitudinal coupling impedance of a hole is the main goal of this work. Since the exact solution is hard to obtain, we try to find an approximate solution based on a variational principle. This approximate solution should be valid in a wide frequency range.

As mentioned in the previous section, a hole cut in the surface imposes a fundamental problem in vector diffraction theory. Relevant theorems and formalism for our impedance problem are summarized in Chapter 2. In Chapter 3, the most important 
in the dissertation, we apply diffraction theorems to a particularly simple geometry, namely, a hole in an infinitely flat screen. Although accelerator beam pipe is torus in shape with circular, elliptical, or rectangular cross section, we consider the beam pipe with rectangular cross section because other shapes raise the question of curvature effect of a hole which is not essential to our problem. Analytic results and comparison with numerical estimates are presented in Chapter 4. Conclusions and suggestions for a further investigation are presented in Chapter 5 . 


\section{Chapter 2}

\section{Vector Diffraction Theory}

\subsection{Introduction}

The diffraction problem deals with the interaction between waves of finite wavelengths and obstacles. A geometric theory describing the diffraction pattern was developed by Huygens and Fresenel whose idea was put forward in the mathematical form by Kirchhoff. Kirchhoff's scalar diffraction theory is based on the integral equation whose solution is in general impossible to find, and hence subject to the various approximations for solutions. When Kirchhoff applied his formula to the problem of diffraction by the black (or opaque) screen with an aperture in it, he assumed that [16]:

1. A field function $(\psi)$ and its normal derivative $\left(\frac{\partial \psi}{\partial n}\right)$ on the screen vanish except in the aperture.

2. The values of $\psi$ and $\frac{\partial \psi}{\partial n}$ in the aperture are equal to the values of the incident waves in the absence of any screen or obstacles.

It is these approximations that contain mathematical inconsistencies and physical deficiencies and not the Kirchhoff integral equation itself [2]. 
Since Kirchhoff's integral equation is based on the scalar Green's function, it does not satisfy Maxwell's equations in general. A vector analog of Kirchhoff's integral equation was developed by Stratton and Chu [27] which requires not only the tangential electric and magnetic fields but also the normal electric fields on the entire boundary surface surrounding the region of interest where the fields are to be computed. The choice of vector Green's function is rather arbitrary. The proper choice of Green's function will remove the normal electric fields from the integral equation [22].

Green's function, which relates the vector fields and the vector sources, should be in general dyadic or tensor quantity. In particular, when we want to compute the fields directly by solving vector wave equations for fields instead of resorting to the use of vector potential, the dyadic Green's function has a considerable advantage over a vector Green's function in simplifying the notation. Levine and Schwinger [21] used the dyadic notation on the theory of electromagnetic wave diffaction by an aperture in an infinite plane conducting screen. Employing variational principles, they calculated the transmission coefficient with a great accuracy comparable to the rigorous solution obtained by solving partial differential equations analytically. This is the motivation for using the dyadic Green's function throughout our work.

In Section 2.2, we explain the field equivalence principle which is helpful in understanding the physics involved in the diffraction phenomena. The brief introduction of dyadic Green's function which will be used later is laid out in Sections 2.3 and 2.4. The field equivalence principle will emerge as a natural part of the theory of dyadic Green's function. In Section 2.5, we introduce the Rumsey's reaction concept which not only simplifies the notation but also proves to be useful in deriving the 
variational formula for various quantities of interest. In Section 2.6, we calculate the transmission coefficient of a plane wave incident on the infinite plane with an aperture using all the concepts and formalisms laid out in the previous sections.

\subsection{Field Equivalence Principle}

Various field equivalence principles provide different ways of formulating a boundaryvalue problem $[9,14,24,25,26]$. As an example, consider a solution of Poisson's equation in electrostatic problems. The region $V$ surrounded by the surface $S$ contains no charge. Applying Green's theorem, one can determine uniquely the potential in the region $V$ due to the charge distribution external to $S$ by specifying a surface charge and a dipole layer density on the surface $S$. This illustrates one form of equivalence principle, for we replaced the original volume source with the equivalent surface sources on the mathematical boundary.

A simple application of the equivalence principle for the time-varying electromagnetic fields is illustrated in Fig. 2.1. The sources for an electromagnetic field are contained in a volume $V_{1}$ bounded by a smooth closed surface $S$ as shown in Fig. 2.1a. Suppose we only need to evaluate the fields in the region $V$ bounded by both the surface $S$ and the surface at infinity $S_{\infty}$. For this purpose, assume the original field in $V$ and the null field in $V_{1}$ as shown in Fig. 2.1b. Since the fields change discontinuously across $S$ in Fig. 2.1b, there must exist surface currents to satisfy the boundary conditions:

$$
\mathbf{J}=\mathbf{H} \times \mathbf{n}, \quad \mathbf{J}_{m}=\mathbf{n} \times \mathbf{E},
$$

where $\mathbf{J}$ and $\mathbf{J}_{m}$ denote the electric and magnetic currents respectively, and $\mathbf{n}$ is the unit vector outwardly normal from the region where the fields are to be calculated. 
(This convention is used for the direction of unit normal vector throughout this work.) The integrated effect of these surface currents will uniquely determine the field in the region $V$. This is called Love's field equivalence principle. Evidently this does not make the problem any easier to solve since we do not know the equivalent surface currents until the problem is solved. Since we know that the field can be uniquely determined by the tangential components of $\mathbf{E}$-field or $\mathbf{H}$-field on the surface alone [28], we may modify Love's field equivalence principle such that it requires only magnetic (tangential E) or electric currents (tangential $\mathbf{H}$ ).

Since the field in the region $V_{1}$ is zero, we may place a perfect electric conductor over $S$ without affecting the field in $V_{1}$. Over this conducting surface we have equivalent current source $\mathbf{J}$ and $\mathbf{J}_{m}$. The tangential $\mathbf{E}$-field reduces to zero on the conductor surface which crosses the magnetic current sheet, and the insertion of conductor does not affect the field in the region $V$ due to $\mathbf{J}_{m}$. It can be shown also that an electric current just in front of an electric conductor produces a null field [9]. The field in the region $V$ is then uniquely determined by the magnetic surface current (tangential $\mathbf{E}$ ) adjacent to the perfect electric conductor whose surface coincides with the $S$. In Green's function technique, this is equivalent to finding Green's function to satisfy the boundary conditions for the perfect electric conductor. Similarly, if we place the perfect magnetic conductor on $S$, we only need the electric surface currents (tangential $\mathbf{H})$ to determine the field uniquely in the region $V$. This modification to Love's field equivalence principle is called Schelkunoff's field equivalence principle and is illustrated in Fig. 2.2. 

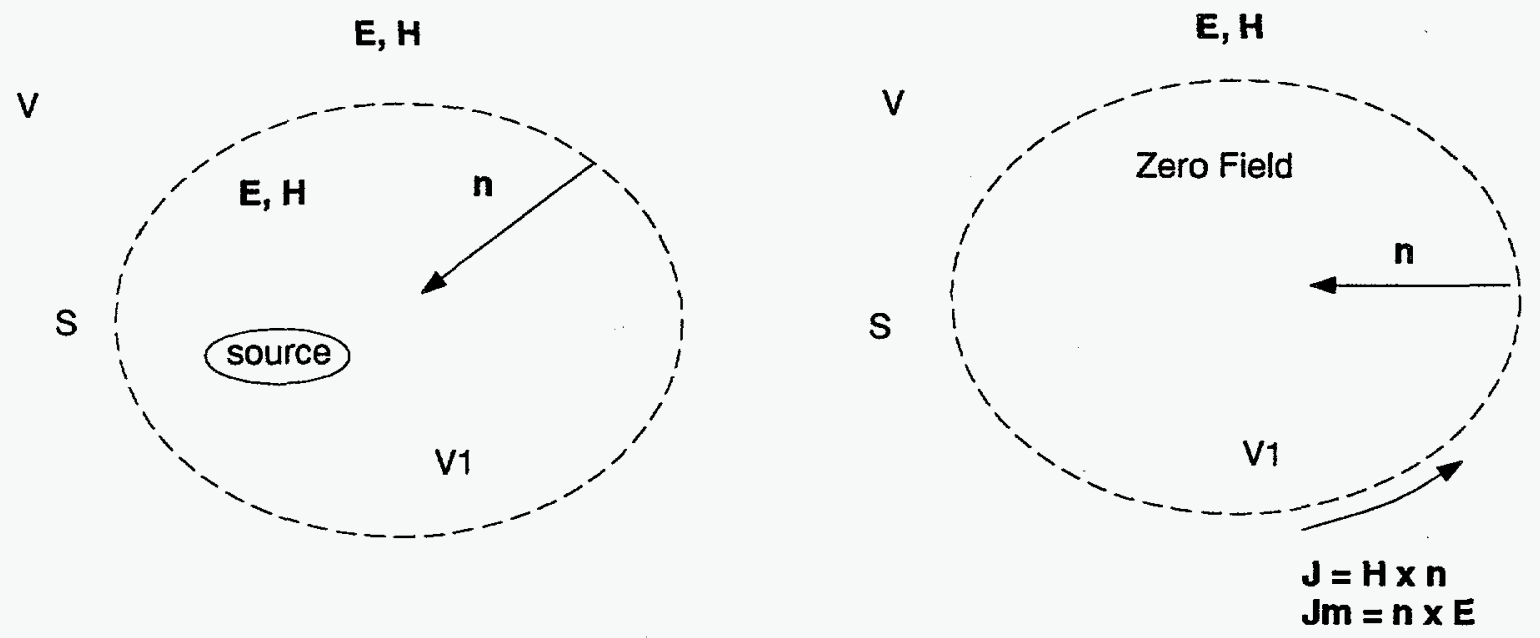

(a)

(b)

Figure 2.1: Illustration of Love's field equivalence principle. (a) Original problem; (b) equivalent to (a).

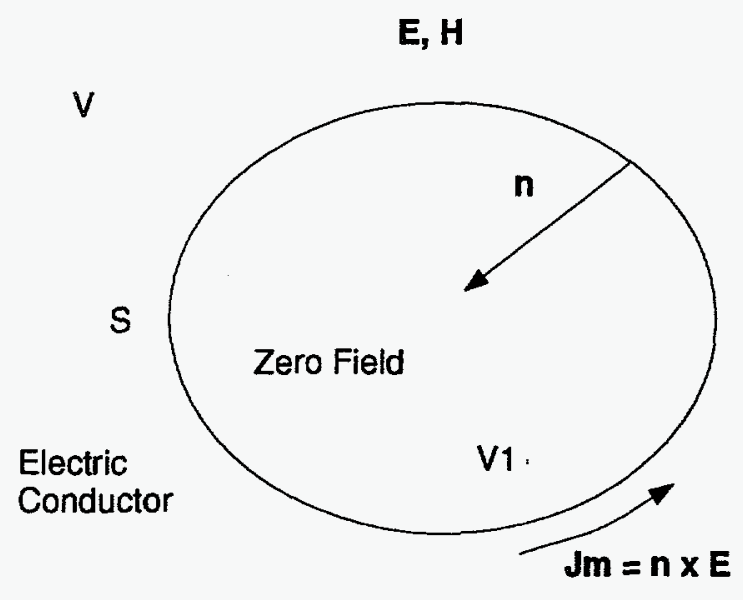

(a)

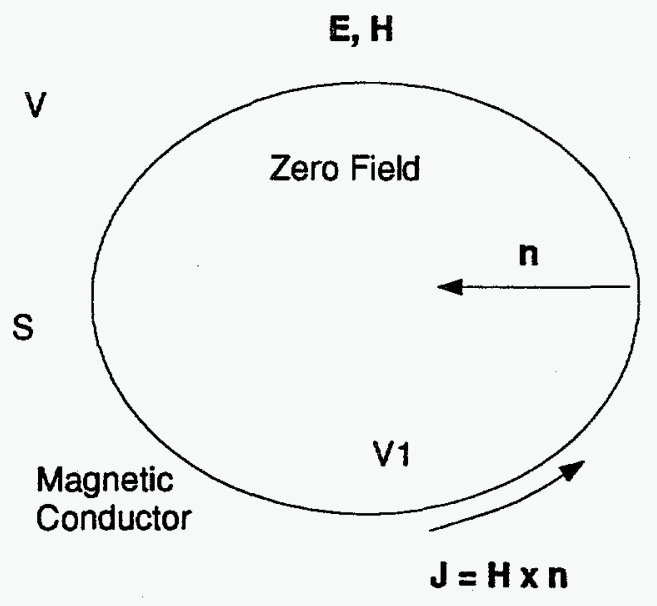

(b)

Figure 2.2: Illustration of Schelkunoff's field equivalence principle. (a) magnetic current over electric conductor; (b) electric current over magnetic conductor. 


\subsection{Free-Space Dyadic Green's Function}

Maxwell's equations in the phasor form are

$$
\begin{aligned}
\nabla \times \mathbf{E}+j \omega \mu \mathbf{H} & =-\mathbf{J}_{m} \\
\nabla \times \mathbf{H}-j \omega \epsilon \mathbf{E} & =\mathbf{J} \\
\nabla \cdot \mathbf{H} & =\rho_{m} / \mu, \\
\nabla \cdot \mathbf{E} & =\rho / \epsilon .
\end{aligned}
$$

It is assumed that all quantities vary as $e^{j \omega t}$. Quantities $\mathbf{J}_{m}$ and $\rho_{m}$ are densities of magnetic current and magnetic charge, respectively. Currents and charges are related by the equation of continuity,

$$
\begin{aligned}
\nabla \cdot \mathbf{J}+j \omega \rho & =0, \\
\nabla \cdot \mathbf{J}_{m}+j \omega \rho_{m} & =0 .
\end{aligned}
$$

The vector wave equation for $\mathbf{E}$ is obtained by taking curl of curl $\mathbf{E}$ resulting in

$$
\nabla \times \nabla \times \mathbf{E}-k^{2} \mathbf{E}=-j \omega \mu \mathbf{J}-\nabla \times \mathbf{J}_{m},
$$

where $k^{2}=\omega^{2} \mu \epsilon$. In a similar fashion we find that $\mathbf{H}$ is a solution of

$$
\nabla \times \nabla \times \mathbf{H}-k^{2} \mathbf{H}=-j \omega \epsilon \mathbf{J}_{m}+\nabla \times \mathbf{J}
$$

We can derive either Eq. (2.7) or (2.8) from the other using the substitutions $\mathbf{E} \rightarrow \mathbf{H}$, $\mathbf{H} \rightarrow-\mathbf{E}, \mathbf{J} \rightarrow \mathbf{J}_{m}, \mathbf{J}_{m} \rightarrow-\mathbf{J}$, and $\epsilon \leftrightarrow \mu$.

Both $\mathbf{E}$ and $\mathbf{H}$ fields satisfy the linear vector equation in the form

$$
\mathcal{L} \mathbf{f}=\mathrm{g} .
$$


We first note that, in general, the solution cannot be expressed in terms of a single scalar Green's function, as, for example,

$$
\mathbf{f}(\mathbf{r})=\int_{V} G\left(\mathbf{r} \mid \mathbf{r}^{\prime}\right) \mathbf{g}\left(\mathbf{r}^{\prime}\right) d V^{\prime}
$$

Such a relationship implies that the source $g\left(\mathbf{r}^{\prime}\right)$ everywhere parallel to the $x$ axis generates field $\mathbf{f}(\mathbf{r})$ parallel to the same axis. This is true for the relationship between the current source and the magnetic vector potential but not for the fields. It is therefore necessary to use nine scalar Green's functions to express the three components of $\mathbf{f}(\mathbf{r})$ in terms of three components of the source $g\left(\mathbf{r}^{\prime}\right)$. One of such nine scalar Green's functions, $G_{x y}\left(\mathbf{r} \mid \mathbf{r}^{\prime}\right)$, measures the $x$-component of the field at $\mathbf{r}$ due to a unit $\mathrm{y}$-directed source at $\mathbf{r}^{\prime}$.

Thus the equations for $\mathbf{f}(\mathbf{r})$ can be written as [6]

$$
\mathbf{f}(\mathbf{r})=\int\left(\mathbf{G}_{x} \mathbf{e}_{x} \cdot \mathbf{g}+\mathbf{G}_{y} \mathbf{e}_{y} \cdot \mathbf{g}+\mathbf{G}_{z} \mathbf{e}_{z} \cdot \mathbf{g}\right) d V^{\prime}=\int \overline{\mathbf{G}} \cdot \mathbf{g} d V^{\prime}
$$

where $\overline{\mathbf{G}}$ is the dyadic Green's function defined as

$$
\overline{\mathbf{G}}=\mathbf{G}_{x} \mathbf{e}_{x}+\mathbf{G}_{y} \mathbf{e}_{y}+\mathbf{G}_{z} \mathbf{e}_{z} \text {. }
$$

(In this work, bold characters are used to denote vectors and bold characters with a bar to denote dyadic quantities.) The G's are the column vector of the dyadic Green's function $\overline{\mathbf{G}}$, for example, as

$$
\mathrm{G}_{x}=G_{x x} \mathbf{e}_{x}+G_{y x} \mathbf{e}_{y}+G_{z x} \mathbf{e}_{z}
$$

In the matrix notation, Eq. (2.10) may be rewritten as

$$
\left[\begin{array}{l}
d f_{x} \\
d f_{y} \\
d f_{z}
\end{array}\right]=\left[\begin{array}{lll}
G_{x x} & G_{x y} & G_{x z} \\
G_{y x} & G_{y y} & G_{y z} \\
G_{z x} & G_{z y} & G_{z z}
\end{array}\right]\left[\begin{array}{l}
d g_{x} \\
d g_{y} \\
d g_{z}
\end{array}\right],
$$


or in the tensor notation,

$$
f_{i}=\int G_{i j} g_{j} d V^{\prime} .
$$

Consider the vector wave equation for $\mathbf{E}$ in the free space excited by the electric current,

$$
\nabla \times \nabla \times \mathbf{E}-k^{2} \mathbf{E}=-j \omega \mu \mathbf{J} .
$$

The dyadic Green's function of electric type is defined as a solution of

$$
\nabla \times \nabla \times \overline{\mathbf{G}}_{e}-k^{2} \overline{\mathbf{G}}_{e}=\overline{\mathbf{I}} \delta\left(\mathbf{r}-\mathbf{r}^{\prime}\right)
$$

where $\overline{\mathbf{I}}$ is the unit dyadic defined as

$$
\overline{\mathbf{I}}=\mathbf{e}_{x} \mathbf{e}_{x}+\mathbf{e}_{y} \mathbf{e}_{y}+\mathbf{e}_{z} \mathbf{e}_{z} .
$$

Subscript $e$ of $\overline{\mathbf{G}}_{e}$ denotes the Green's function of electric type. We do not impose any boundary conditions on $\overline{\mathbf{G}}_{e}$ except that it should satisfy the radiation condition at infinity.

Following Levine and Schwinger [21], we transform Eq. (2.16) to the vector Helmholtz wave equation form. A vector identity

$$
\nabla \times \nabla \times=\nabla \nabla \cdot-\nabla^{2}
$$

can be used to obtain

$$
\left(\nabla^{2}+k^{2}\right) \overline{\mathbf{G}}_{e}\left(\mathbf{r} \mid \mathbf{r}^{\prime}\right)=-\left(\overline{\mathbf{I}}+\frac{1}{k^{2}} \nabla \nabla\right) \delta\left(\mathbf{r}-\mathbf{r}^{\prime}\right)
$$

Since we know that, in free space, the scalar Green's function $G\left(\mathbf{r} \mid \mathbf{r}^{\prime}\right)$ obeys the relation

$$
\left(\nabla^{2}+k^{2}\right) G\left(\mathbf{r} \mid \mathbf{r}^{\prime}\right)=-\delta\left(\mathbf{r}-\mathbf{r}^{\prime}\right)
$$


the corresponding dyadic Green's function should be in the form

$$
\overline{\mathbf{G}}_{e}\left(\mathbf{r} \mid \mathbf{r}^{\prime}\right)=\left(\overline{\mathbf{I}}+\frac{1}{k^{2}} \nabla \nabla\right) G
$$

The scalar Green's function satisfying the radiation condition is well known as

$$
G\left(\mathbf{r} \mid \mathbf{r}^{\prime}\right)=G(R)=\frac{e^{-j k R}}{4 \pi R}
$$

where $R=|\mathbf{R}|=\left|\mathbf{r}-\mathbf{r}^{\prime}\right|$ is the distance between the source point $\mathbf{r}^{\prime}$ and the field point $\mathbf{r}$. Thus, the dyadic Green's function is

$$
\overline{\mathbf{G}}_{e}\left(\mathbf{r} \mid \mathbf{r}^{\prime}\right)=\left(\overline{\mathbf{I}}+\frac{1}{k^{2}} \nabla \nabla\right) \frac{e^{-j k R}}{4 \pi R} .
$$

The hierarchy of the above dyadic Green's function becomes clear by constructing an explicit coordinate-free form [8]. In order to do that we will use the following relationships

$$
\begin{aligned}
\nabla R & =\hat{\mathbf{R}} \\
\nabla \hat{\mathbf{R}} & =\frac{1}{R}(\overline{\mathbf{I}}-\hat{\mathbf{R}} \hat{\mathbf{R}})
\end{aligned}
$$

where $\mathbf{R}=\mathbf{r}-\mathbf{r}^{\prime}$ and $\hat{\mathbf{R}}=\mathbf{R} / R$.

Using the chain rule that if $f(u)$ is a function of $u$, and $u$ is a function of $\mathbf{r}$, we have

$$
\nabla f=\frac{d f}{d u} \nabla u
$$

With the aid of the above relations, we obtain

$$
\nabla \frac{e^{-j k R}}{R}=-\left(j k+\frac{1}{R}\right) \frac{e^{-j k R}}{R} \hat{\mathbf{R}}
$$

and

$$
\nabla \nabla \frac{e^{-j k R}}{R}=\left[-\left(\frac{j k}{R}+\frac{2}{R^{2}}\right)(\overline{\mathbf{I}}-3 \hat{\mathbf{R}} \hat{\mathbf{R}})-k^{2} \hat{\mathbf{R}} \hat{\mathbf{R}}\right] \frac{e^{-j k R}}{R}
$$


Substituting Eq. (2.26) into Eq. (2.22), we finally obtain

$$
\overline{\mathbf{G}}_{e}\left(\mathbf{r} \mid \mathbf{r}^{\prime}\right)=(\overline{\mathbf{I}}-\hat{\mathbf{R}} \hat{\mathbf{R}}) G(R)-\frac{j}{k R}(\overline{\mathbf{I}}-3 \hat{\mathbf{R}} \hat{\mathbf{R}}) G(R)-\frac{1}{k^{2} R^{2}}(\overline{\mathbf{I}}-3 \hat{\mathbf{R}} \hat{\mathbf{R}}) G(R) .
$$

The first term, which varies as $1 / R$, is the radiation term which is purely transverse to the direction $\mathbf{R}$ as the dyad $\overline{\mathbf{I}}-\hat{\mathbf{R}} \hat{\mathbf{R}}$ projects any vector perpendicular to $\hat{\mathbf{R}}$. Thus the radiation field far from the source is a plane wave. The second and third terms are also familiar induction and electrostatic terms, respectively. For an arbitrary current distribution, the electric field will be

$$
\mathbf{E}(\mathbf{r})=-j \omega \mu \int \overline{\mathbf{G}}_{e}\left(\mathbf{r} \mid \mathbf{r}^{\prime}\right) \cdot \mathbf{J}\left(\mathbf{r}^{\prime}\right) d V^{\prime}
$$

Similarly for $\mathbf{H}$ fields due to the magnetic currents satisfying

$$
\nabla \times \nabla \times \mathbf{H}-k^{2} \mathbf{H}=-j \omega \epsilon \mathbf{J}_{m}
$$

the dyadic Green's function of magnetic type is defined as the solution of

$$
\nabla \times \nabla \times \overline{\mathbf{G}}_{m}-k^{2} \overline{\mathbf{G}}_{m}=\overline{\mathbf{I}} \delta\left(\mathbf{r}-\mathbf{r}^{\prime}\right)
$$

For an arbitrary magnetic current distribution the magnetic field will be

$$
\mathbf{H}(\mathbf{r})=-j \omega \epsilon \int \overline{\mathbf{G}}_{m}\left(\mathbf{r} \mid \mathbf{r}^{\prime}\right) \cdot \mathbf{J}_{m}\left(\mathbf{r}^{\prime}\right) d V^{\prime}
$$

$\overline{\mathbf{G}}_{m}$ is obviously equal to $\overline{\mathbf{G}}_{e}$, and we denote both as $\overline{\mathbf{G}}_{\mathbf{0}}$, which is called the freespace dyadic Green's function. In general the dyadic Green's functions of electric type and magnetic type are different from each other when the explicit boundary conditions are included in the definition of Green's function. For example, in the region bounded by a perfectly conducting wall, the boundary conditions on the surface satisfied by the Green's function are such that

$$
\mathbf{n} \times \overline{\mathbf{G}}_{e}=0, \quad \mathbf{n} \times \nabla \times \overline{\mathbf{G}}_{m}=0
$$


which says that the tangential electric field on the electric conductor vanishes. Thus $\overline{\mathbf{G}}_{e} \neq \overline{\mathbf{G}}_{m}$ in general.

From Eq. (2.27), we see that the free-space dyadic Green's function has symmetrical properties,

$$
\overline{\mathbf{G}}_{0}\left(\mathbf{r} \mid \mathbf{r}^{\prime}\right)=\overline{\mathbf{G}}_{0}^{T}\left(\mathbf{r}^{\prime} \mid \mathbf{r}\right), \quad \overline{\mathbf{G}}_{0}\left(\mathbf{r} \mid \mathbf{r}^{\prime}\right)=\overline{\mathbf{G}}_{0}\left(\mathbf{r}^{\prime} \mid \mathbf{r}\right),
$$

where superscript $T$ denotes the transpose of a dyadic. The first identity, often called reciprocity relation, is satisfied by all types of Green's function but the second one is special to the free-space case. These are useful because we can write

$$
\overline{\mathbf{G}}_{0} \cdot \mathbf{J}=\mathbf{J} \cdot \overline{\mathbf{G}}_{0}
$$

without worrying about ordering of the multiplication, i.e., $\overline{\mathbf{G}}_{0}$ commutes with an arbitrary vector.

\subsection{Dyadic Green's Function and Field Equiva- lence Principle}

Consider the source in the region $V_{1}$ shown in Fig. 2.1a bounded by the surface $S$. The fields of physical interest are those contained within the regions devoid of charges and currents. Such a region is denoted as $V$ in Fig. 2.1a bounded by $S$ and the surface at infinity $S_{\infty}$. The unit vector $\mathbf{n}$ is normal to $S$ outward from the region $V$. Then the electric field in $V$ satisfies the source-free wave equation,

$$
\nabla \times \nabla \times \mathbf{E}-k^{2} \mathbf{E}=0 .
$$

We define the dyadic Green's function as a solution of

$$
\nabla \times \nabla \times \overline{\mathbf{G}}-k^{2} \overline{\mathbf{G}}=\overline{\mathbf{I}} \delta\left(\mathbf{r}-\mathbf{r}^{\prime}\right)
$$


This Green's function is defined in the unbounded space, $V+V_{1}$, and it should satisfy the radiation condition. This is essentially the free-space dyadic Green's function previously denoted as $\overline{\mathbf{G}}_{0}$ but, since we will impose a boundary condition later, we will leave it as $\overline{\mathbf{G}}$.

In order to relate the field in $V$ with the surface integral over $S$, we use the Green's second vector identity written as

$$
\int_{S+S_{\infty}} d S \mathbf{n} \cdot[\mathbf{B} \times(\nabla \times \mathbf{A})-\mathbf{A} \times(\nabla \times \mathbf{B})]=\int_{V} d V[\mathbf{A} \cdot \nabla \times(\nabla \times \mathbf{B})-\mathbf{B} \cdot \nabla \times(\nabla \times \mathbf{A})] .
$$

Substituting $\mathbf{A}\left(\mathbf{r}^{\prime}\right)=\mathbf{E}\left(\mathbf{r}^{\prime}\right)$ and $\mathbf{B}\left(\mathbf{r}^{\prime}\right)=\overline{\mathbf{G}}\left(\mathbf{r}^{\prime} \mid \mathbf{r}\right) \cdot \mathbf{e}$, where $\mathbf{e}$ is an arbitrary constant vector, we obtain

$$
\begin{aligned}
& \int_{S+S_{\infty}} d S^{\prime} \mathbf{n} \cdot\left[\left(\overline{\mathbf{G}}\left(\mathbf{r}^{\prime} \mid \mathbf{r}\right) \cdot \mathbf{e}\right) \times\left(\nabla^{\prime} \times \mathbf{E}\left(\mathbf{r}^{\prime}\right)\right)-\mathbf{E}\left(\mathbf{r}^{\prime}\right) \times\left(\nabla^{\prime} \times \overline{\mathbf{G}}\left(\mathbf{r}^{\prime} \mid \mathbf{r}\right) \cdot \mathbf{e}\right)\right] \\
= & \int_{V} d V^{\prime}\left[\mathbf{E}\left(\mathbf{r}^{\prime}\right) \cdot \nabla^{\prime} \times \nabla^{\prime} \times\left(\overline{\mathbf{G}}\left(\mathbf{r}^{\prime} \mid \mathbf{r}\right) \cdot \mathbf{e}\right)-\left(\overline{\mathbf{G}}\left(\mathbf{r}^{\prime} \mid \mathbf{r}\right) \cdot \mathbf{e}\right) \cdot \nabla^{\prime} \times \nabla^{\prime} \times \mathbf{E}\left(\mathbf{r}^{\prime}\right)\right] .
\end{aligned}
$$

Using the relation $\nabla \times \mathbf{E}=-j \omega \mu \mathbf{H}$ and noting that the surface integral vanishes on $S_{\infty}$, we obtain

$$
j \omega \mu \int_{S} d S^{\prime}(\mathbf{n} \times \mathbf{H}) \cdot \overline{\mathbf{G}}\left(\mathbf{r}^{\prime} \mid \mathbf{r}\right)-\int_{S} d S^{\prime}(\mathbf{n} \times \mathbf{E}) \cdot \nabla^{\prime} \times \overline{\mathbf{G}}\left(\mathbf{r}^{\prime} \mid \mathbf{r}\right)=\left\{\begin{array}{ll}
\mathbf{E}(\mathbf{r}) & \text { if } \mathbf{r} \in V \\
0 & \text { otherwise }
\end{array},\right.
$$

where we used Eq. (2.35) and Eq. (2.36) to evaluate the volume integral. Physical interpretation of this will be made clearer by applying the relation $[21,29]$

$$
\nabla \times \overline{\mathbf{G}}_{0}\left(\mathbf{r} \mid \mathbf{r}^{\prime}\right)=\left[\nabla^{\prime} \times \overline{\mathbf{G}}_{0}\left(\mathbf{r}^{\prime} \mid \mathbf{r}\right)\right]^{T}
$$

to see that

$$
\begin{aligned}
\mathbf{E}(\mathbf{r}) & =-j \omega \mu \int_{S} \overline{\mathbf{G}}\left(\mathbf{r} \mid \mathbf{r}^{\prime}\right) \cdot(\mathbf{H} \times \mathbf{n}) d S^{\prime}-\nabla \times \int_{S} \overline{\mathbf{G}}\left(\mathbf{r} \mid \mathbf{r}^{\prime}\right) \cdot(\mathbf{n} \times \mathbf{E}) d S^{\prime} \\
& =\mathbf{E} \text { (due to } \mathbf{J}=\mathbf{H} \times \mathbf{n})+\mathbf{E}\left(\text { due to } \mathbf{J}_{m}=\mathbf{n} \times \mathbf{E}\right)
\end{aligned}
$$


Thus we have proved Love's field equivalence principle. A corresponding expression for the magnetic field may be obtained by substituting $\mathbf{A}\left(\mathbf{r}^{\prime}\right)=\mathbf{H}\left(\mathbf{r}^{\prime}\right)$ and $\mathbf{B}\left(\mathbf{r}^{\prime}\right)=$ $\overline{\mathbf{G}}\left(\mathbf{r}^{\prime} \mid \mathbf{r}\right) \cdot \mathbf{e}$ into Eq. (2.37) and the result is

$$
-j \omega \epsilon \int_{S} d S^{\prime}(\mathbf{n} \times \mathbf{E}) \cdot \overline{\mathbf{G}}\left(\mathbf{r}^{\prime} \mid \mathbf{r}\right)-\int_{S} d S^{\prime}(\mathbf{n} \times \mathbf{H}) \cdot \nabla^{\prime} \times \overline{\mathbf{G}}\left(\mathbf{r}^{\prime} \mid \mathbf{r}\right)= \begin{cases}\mathbf{H}(\mathbf{r}) & \text { if } \mathbf{r} \in V \\ 0 & \text { otherwise }\end{cases}
$$

which is equivalent to replacing $\mathbf{E} \rightarrow \mathbf{H}, \mathbf{H} \rightarrow-\mathbf{E}$, and $\mu \rightarrow \epsilon$ in Eq. (2.39).

If the surface $S$ is a perfectly conducting wall, it will be convenient to distinguish electric and magnetic Green's functions $\overline{\mathbf{G}}_{e}$ and $\overline{\mathbf{G}}_{m}$ which satisfy the boundary conditions

$$
\mathbf{n} \times \overline{\mathbf{G}}_{e}=0, \quad \mathbf{n} \times \nabla \times \overline{\mathbf{G}}_{m}=0 .
$$

The expression for the electric field can be obtained by replacing $\overline{\mathbf{G}}$ with $\overline{\mathbf{G}}_{e}$ in Eq. (2.39), then the first integral vanishes because of the boundary condition. Similarly the magnetic field can be obtained by replacing $\overline{\mathbf{G}}$ with $\overline{\mathbf{G}}_{m}$ in Eq. (2.42), then the second integral vanishes. The resulting expressions are

$$
\begin{aligned}
\mathbf{E}(\mathbf{r}) & =-\int_{S} d S^{\prime}(\mathbf{n} \times \mathbf{E}) \cdot \nabla^{\prime} \times \overline{\mathbf{G}}_{e}\left(\mathbf{r}^{\prime} \mid \mathbf{r}\right) \\
\mathbf{H}(\mathbf{r}) & =-j \omega \epsilon \int_{S} d S^{\prime}(\mathbf{n} \times \mathbf{E}) \cdot \overline{\mathbf{G}}_{m}\left(\mathbf{r}^{\prime} \mid \mathbf{r}\right)
\end{aligned}
$$

Using the symmetrical relations Eq. (2.50) and Eq. (2.53) below, we rewrite the above equations as

$$
\begin{aligned}
\mathbf{E}(\mathbf{r}) & =-\nabla \times \int_{S} d S^{\prime} \overline{\mathbf{G}}_{m}\left(\mathbf{r} \mid \mathbf{r}^{\prime}\right) \cdot(\mathbf{n} \times \mathbf{E}) \\
\mathbf{H}(\mathbf{r}) & =-j \omega \epsilon \int_{S} d S^{\prime} \overline{\mathbf{G}}_{m}\left(\mathbf{r} \mid \mathbf{r}^{\prime}\right) \cdot(\mathbf{n} \times \mathbf{E})
\end{aligned}
$$

which clearly show that the fields are due to the magnetic surface current. We have derived a mathematical expression of the Schelkunoff's field equivalence principle 
shown in Fig. 2.2a. The other one corresponding to Fig. $2.2 \mathrm{~b}$ will also be verified by interchanging the roles of $\overline{\mathbf{G}}_{e}$ and $\overline{\mathbf{G}}_{m}$.

Finally, we summarize the useful symmetrical relations of dyadic Green's functions. The proof is given in Ref. [21, 29].

$$
\begin{gathered}
\nabla \times \nabla \times \overline{\mathbf{G}}-k^{2} \overline{\mathbf{G}}=\overline{\mathbf{I}} \delta\left(\mathbf{r}-\mathbf{r}^{\prime}\right) \\
\mathbf{n} \times \overline{\mathbf{G}}_{1}=0, \mathbf{n} \times \nabla \times \overline{\mathbf{G}}_{2}=0, \quad \mathbf{r} \text { on } \mathrm{S} \\
\overline{\mathbf{G}}\left(\mathbf{r} \mid \mathbf{r}^{\prime}\right)=\left[\overline{\mathbf{G}}\left(\mathbf{r}^{\prime} \mid \mathbf{r}\right)\right]^{T} \\
\nabla \times \overline{\mathbf{G}}_{0}\left(\mathbf{r} \mid \mathbf{r}^{\prime}\right)=\left[\nabla^{\prime} \times \overline{\mathbf{G}}_{0}\left(\mathbf{r}^{\prime} \mid \mathbf{r}\right)\right]^{T} \\
\nabla \times \overline{\mathbf{G}}_{1}\left(\mathbf{r} \mid \mathbf{r}^{\prime}\right)=\left[\nabla^{\prime} \times \overline{\mathbf{G}}_{2}\left(\mathbf{r}^{\prime} \mid \mathbf{r}\right)\right]^{T} \\
\nabla \times \overline{\mathbf{G}}_{2}\left(\mathbf{r} \mid \mathbf{r}^{\prime}\right)=\left[\nabla^{\prime} \times \overline{\mathbf{G}}_{1}\left(\mathbf{r}^{\prime} \mid \mathbf{r}\right)\right]^{T}
\end{gathered}
$$

\subsection{Reaction Concept and Variational Formalism}

Let $\mathbf{E}^{a}$ and $\mathbf{B}^{a}$ be the fields in volume $V$ bounded by a closed surface $S$ and excited by volume distribution of electric current $\mathbf{J}^{a}$ and magnetic current $\mathbf{J}_{m}^{a}$. Similarly for $\mathbf{E}^{b}$ and $\mathbf{H}^{b}$ generated by $\mathbf{J}^{b}$ and $\mathbf{J}_{m}^{b}$. Then, by the Lorentz reciprocity theorem, we get

$$
\oint_{S}\left(\mathbf{E}^{a} \times \mathbf{H}^{b}-\mathbf{E}^{b} \times \mathbf{H}^{a}\right) \cdot d \mathbf{S}=\int_{V}\left(\mathbf{E}^{b} \cdot \mathbf{J}^{a}-\mathbf{H}^{b} \cdot \mathbf{J}_{m}^{a}-\mathbf{E}^{a} \cdot \mathbf{J}^{b}+\mathbf{H}^{a} \cdot \mathbf{J}_{m}^{b}\right) d V
$$

If the current sources are finite and enclosed by the surface $S$, the left side of Eq. (2.54) can be shown to vanish. One of the most useful forms of Lorentz reciprocity theorem, in terms of Rumsey's notation [23], may then be expressed as

$$
<a, b>=<b, a>
$$


where $\langle a, b\rangle$ is called by Rumsey as reaction of the field $a$ onto the current source $b$ and is defined as

$$
<a, b>=\int_{V}\left(\mathbf{E}^{a} \cdot \mathbf{J}^{b}-\mathbf{H}^{a} \cdot \mathbf{J}_{m}^{b}\right) d V .
$$

Rumsey showed that, if the quantity of interest can be expressed as a reaction, a variational approximation with a suitable stationary property can be easily derived. To see that, we let the correct reaction be $\left\langle c_{a}, c_{b}\right\rangle$ and the trial reaction be $\langle a, b\rangle$. Then it is shown that if $\langle a, b\rangle$ satisfies the condition

$$
\left.\left\langle c_{a}, c_{b}\right\rangle \approx<a, b>=<c_{a}, b\right\rangle=\left\langle a, c_{b}\right\rangle
$$

$\langle a, b\rangle$ is a variational approximation to $\left\langle c_{a}, c_{b}\right\rangle$ which is stationary with respect to the correct values of $c_{a}$ and $c_{b}$. In order to prove it, following Harrington [14], we let

$$
a=c_{a}+p_{a} e_{a}, \quad b=c_{b}+p_{b} e_{b}
$$

where $p$ is an arbitrary parameter and $e$ represents an error. Then $\langle a, b\rangle$ becomes

$$
\left.\left.\left.\left.<a, b>=<c_{a}, c_{b}\right\rangle+p_{a}<e_{a}, c_{b}\right\rangle+p_{b}<c_{a}, e_{b}\right\rangle+p_{a} p_{b}<e_{a}, e_{b}\right\rangle
$$

Using Eq. (2.57), we rewrite the above equation as

$$
\left.<a, b>=<c_{a}, c_{b}>-p_{a} p_{b}<e_{a}, e_{b}\right\rangle
$$

Since the equation satisfies

$$
\frac{\partial<a, b>}{\partial p_{a}}=\frac{\partial\langle a, b>}{\partial p_{a}}=0 \text { as } p_{a} \rightarrow 0, p_{b} \rightarrow 0,
$$

we have proved the stationary property of $\langle a, b\rangle$.

Even if Eq. (2.57) leads us to a variational formula, it does not enable us to set up the proper functional equations to be solved when a trial field and associated source 
are expanded in series of functions with unknown coefficients. For that purpose we will use the following expression,

$$
<c_{a}, c_{b}>\approx<a, b>=\frac{\left\langle a, c_{b}><c_{a}, b>\right.}{<a, b>}
$$

which is the same as Eq. (2.57). By equating these two expressions, we are ready to solve for unknown coefficients, which is usually equivalent to solving linear algebraic equations. In the final stage, by substituting the calculated coefficients into Eq. (2.57), we will have desired results which should be a good approximation to the correct solution.

\subsection{Application to Aperture Problem in an Infi- nite Screen}

We consider a surface $S$ which consists of an infinitely thin, perfectly conducting plane

screen $S_{2}$ of infinite extent and an aperture $S_{1}$ (or $S_{a}$ ), which is shown in Fig. 2.3.

If we denote the fields as $\mathbf{E}_{0}(\mathbf{r}), \mathbf{H}_{0}(\mathbf{r})$ in the absence of an aperture, we may write the fields in each half-space as

$$
\begin{array}{lll}
\mathbf{E}(\mathbf{r})=\mathbf{E}_{0}(\mathbf{r})+\mathbf{E}_{1}(\mathbf{r}), & \mathbf{H}(\mathbf{r})=\mathbf{H}_{0}(\mathbf{r})+\mathbf{H}_{1}(\mathbf{r}), & z \leq 0 \\
\mathbf{E}(\mathbf{r})=\mathbf{E}_{2}(\mathbf{r}), & \mathbf{H}(\mathbf{r})=\mathbf{H}_{2}(\mathbf{r}), & z \geq 0
\end{array}
$$

where the subscripts 1 and 2 indicate the region $z \leq 0$ and $z \geq 0$, respectively, and the fields are subject to the boundary condition

$$
\mathbf{e}_{z} \times \mathbf{E}=0, \quad \mathbf{e}_{z} \cdot \mathbf{H}=0, \mathbf{r} \text { on } S_{2}
$$

$\mathbf{E}_{0}$ and $\mathbf{H}_{0}$ can be decomposed as

$$
\mathbf{E}_{0}=\mathbf{E}^{i n c}+\mathbf{E}^{r e f}, \mathbf{H}_{0}=\mathbf{H}^{i n c}+\mathbf{H}^{r e f}
$$




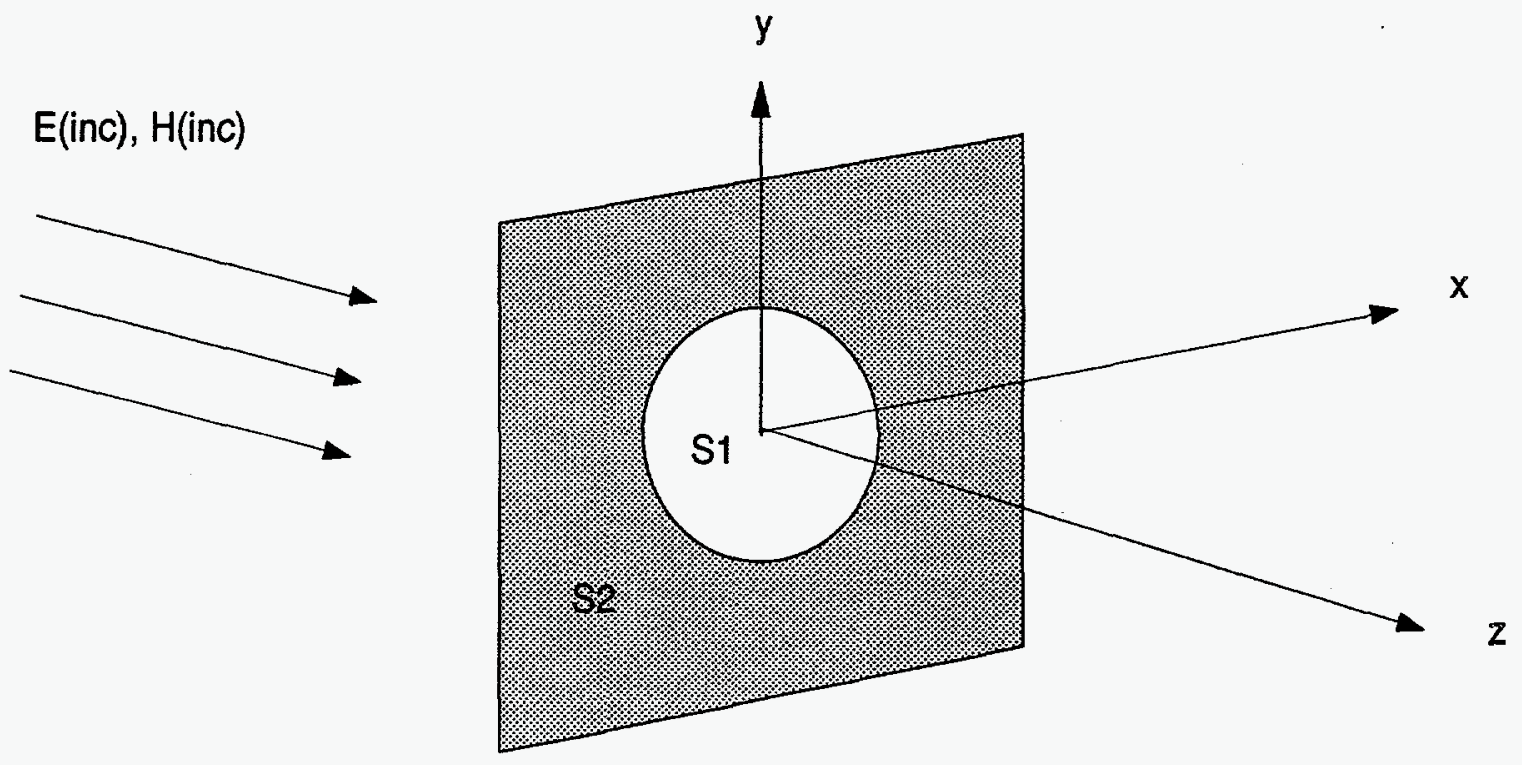

Figure 2.3: Diffracting aperture in a plane screen.

where the superscripts inc and ref indicate the incident and reflected waves from the plane conducting screen which is not perforated. The boundary conditions on the surface $S$ are

$$
E_{z}^{i n c}=E_{z}^{r e f}, H_{t}^{i n c}=H_{t}^{r e f}, \mathbf{r} \text { on } S
$$

where the subscripts $z$ and $t$ denote the $z$-component (normal component) and the tangential component of fields, respectively.

The boundary conditions in the apertures can be expressed by the continuity of fields in the aperture as

$$
\begin{aligned}
& E_{1 t}=E_{2 t}, \quad H_{2 t}-H_{1 t}=H_{0 t}, \quad \mathbf{r} \text { in } S_{a}, \\
& H_{1 z}=H_{2 z}, \quad E_{2 z}-E_{1 z}=E_{0 z}, \quad \mathbf{r} \text { in } S_{a},
\end{aligned}
$$

which are satisfied if

$$
H_{2 t}=-H_{1 t}=\frac{1}{2} H_{0 t}=H_{t}^{i n c}, \mathbf{r} \text { in } S_{a}
$$




$$
E_{2 z}=-E_{1 z}=\frac{1}{2} E_{0 z}=E_{z}^{i n c}, \quad \mathbf{r} \text { in } S_{a} .
$$

Using Eq. (2.46) and Eq. (2.47), we express the fields in each half-space separated by the screen in the integral form as

$$
\left.\begin{array}{r}
\mathbf{E}(\mathbf{r})=\nabla \times \int_{S_{a}} \overline{\mathbf{G}}_{m}^{+}\left(\mathbf{r} \mid \mathbf{r}^{\prime}\right) \cdot\left(\mathbf{e}_{z} \times \mathbf{E}\right) d S^{\prime} \\
\mathbf{H}(\mathbf{r})=j \omega \epsilon \int_{S_{a}} \overline{\mathbf{G}}_{m}^{+}\left(\mathbf{r} \mid \mathbf{r}^{\prime}\right) \cdot\left(\mathbf{e}_{z} \times \mathbf{E}\right) d S^{\prime}
\end{array}\right\} z \geq 0,
$$

where the superscripts + and - denote the region $z \geq 0$ and $z \leq 0$, respectively. Care must be taken with the sign of unit vector $\mathbf{n}$. Following our convention that the direction of $\mathbf{n}$ is outwardly normal from the region where the fields are to be calculated, $\mathbf{n}=-\mathbf{e}_{z}$ for the region $z \geq 0$ and $\mathbf{n}=\mathbf{e}_{z}$ for the region $z \leq 0$.

The dyadic Green's function for the half-space can be readily constructed using the image principle and is shown to be $[21,29]$

$$
\overline{\mathbf{G}}_{e, m}^{+}\left(\mathbf{r} \mid \mathbf{r}^{\prime}\right)=\overline{\mathbf{G}}_{\mathbf{0}}\left(\mathbf{r} \mid \mathbf{r}^{\prime}\right) \mp \overline{\mathbf{G}}_{0}\left(\mathbf{r} \mid \mathbf{r}^{\prime}-2 \mathbf{e}_{z} \mathbf{e}_{z} \cdot \mathbf{r}^{\prime}\right) \cdot\left(\overline{\mathbf{I}}-2 \mathbf{e}_{z} \mathbf{e}_{z}\right), \quad z, z^{\prime} \geq 0,
$$

where the upper and lower signs are employed for $\overline{\mathbf{G}}_{e}^{+}$and $\overline{\mathbf{G}}_{m}^{+}$, respectively, and

$$
\overline{\mathbf{G}}^{-}\left(\mathbf{r} \mid \mathbf{r}^{\prime}\right)=\overline{\mathbf{G}}^{+}\left(\mathbf{r}-2 \mathbf{e}_{z} \mathbf{e}_{z} \cdot \mathbf{r}, \mathbf{r}^{\prime}-2 \mathbf{e}_{z} \mathbf{e}_{z} \cdot \mathbf{r}^{\prime}\right), \quad z, z^{\prime} \leq 0 .
$$

If Eq. (2.73) is multiplied from the right by the vector $\mathbf{e}_{z} \times \mathbf{E}\left(\mathbf{r}^{\prime}\right)$ and the result evaluated at $z^{\prime}=0$, it can be seen that

$$
\overline{\mathbf{G}}_{m}^{+}\left(\mathbf{r} \mid \mathbf{r}^{\prime}\right) \cdot\left[\mathbf{e}_{z} \times \mathbf{E}\left(\mathbf{r}^{\prime}\right)\right]=2 \overline{\mathbf{G}}_{0}\left(\mathbf{r} \mid \mathbf{r}^{\prime}\right) \cdot\left[\mathbf{e}_{z} \times \mathbf{E}\left(\mathbf{r}^{\prime}\right)\right]
$$

Thus, Eqs. (2.71) and (2.72) are rewritten in the form

$$
\left.\begin{array}{r}
\mathbf{E}(\mathbf{r})=\nabla \times \int_{S_{a}} \overline{\mathbf{G}}_{0}\left(\mathbf{r} \mid \mathbf{r}^{\prime}\right) \cdot\left(2 \mathbf{e}_{z} \times \mathbf{E}\right) d S^{\prime} \\
\mathbf{H}(\mathbf{r})=j \omega \epsilon \int_{S_{a}} \overline{\mathbf{G}}_{0}\left(\mathbf{r} \mid \mathbf{r}^{\prime}\right) \cdot\left(2 \mathbf{e}_{z} \times \mathbf{E}\right) d S^{\prime}
\end{array}\right\} z \geq 0,
$$




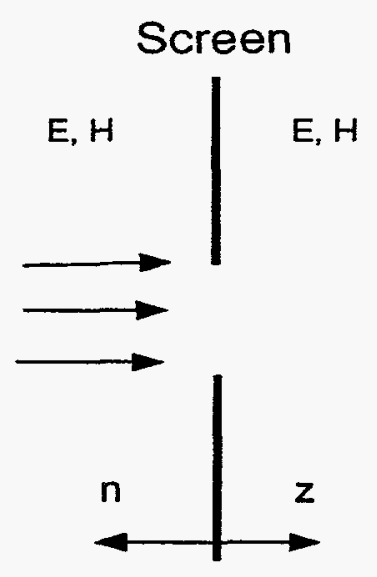

(a)

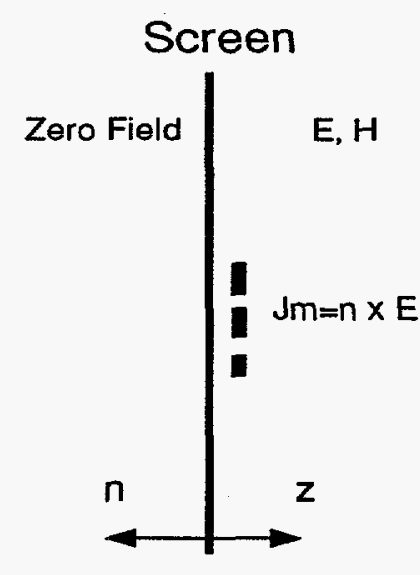

(b)

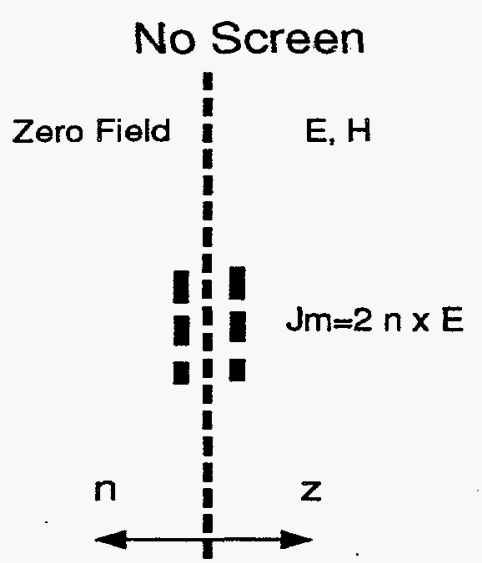

(c)

Figure 2.4: (a) Original problem; (b) and (c) Equivalent problem to (a) for the region $z \geq 0$.

The physical steps hidden in the derivation can be visualized from the field equivalence principle shown in Figs. 2.4 and 2.5 for the regions $z \geq 0$ and $z \leq 0$, respectively.

If we know the tangential $\mathbf{E}$-fields in the aperture, we can calculate the fields everywhere using Eqs. (2.76) and (2.77). The desired integral equation for the tangential E-field in the aperture can be written by noting the boundary conditions Eqs. (2.69) and (2.70). In the plane of the aperture, we have

$$
\left.\begin{array}{l}
\mathbf{e}_{z} \cdot \mathbf{E}^{i n c}(\mathbf{r})=\mathbf{e}_{z} \cdot \nabla \times \int_{S_{a}} \overline{\mathbf{G}}_{0}\left(\mathbf{r} \mid \mathbf{r}^{\prime}\right) \cdot\left(2 \mathbf{e}_{z} \times \mathbf{E}\right) d S^{\prime} \\
\mathbf{e}_{z} \times \mathbf{H}^{i n c}(\mathbf{r})=j \omega \epsilon \mathbf{e}_{z} \times \int_{S_{a}} \overline{\mathbf{G}}_{\mathbf{0}}\left(\mathbf{r} \mid \mathbf{r}^{\prime}\right) \cdot\left(2 \mathbf{e}_{z} \times \mathbf{E}\right) d S^{\prime}
\end{array}\right\} \mathbf{r}, \mathbf{r}^{\prime} \text { in } S_{a}
$$

In general, it is difficult if not impossible to solve this integral equation. Fortunately, most of the physically interesting quantities such as the transmission coefficient do not require detailed knowledge of the field around the obstacle. Besides, such a quantity is expressed in an integral form which is amenable to an approximation. We take the calculation of the transmission coefficient through a circular aperture as an example of using variational formalism to obtain the approximate solution.

Consider a linearly polarized plane wave normally incident on an aperture in a 


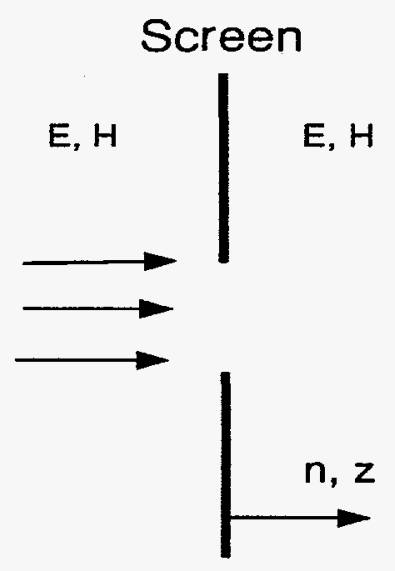

(a)

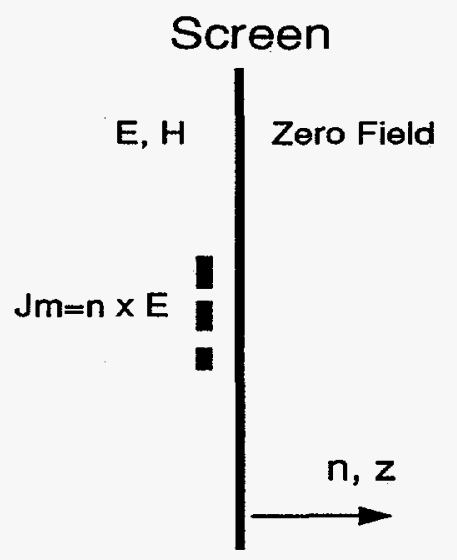

(b)

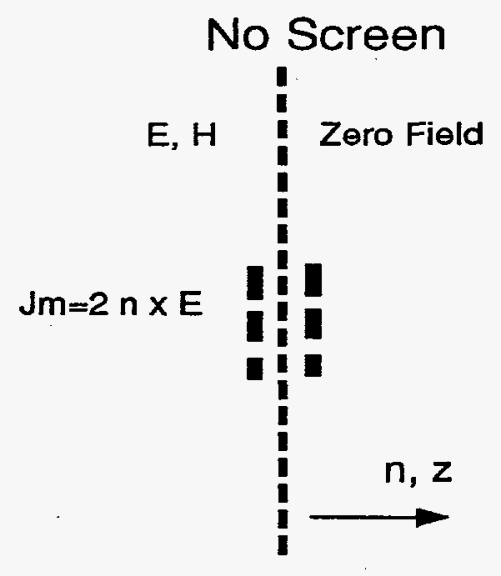

(c)

Figure 2.5: (a) Original problem; (b) and (c) Equivalent problem to (a) for the region $z \leq 0$.

plane screen. The transmission coefficient is defined as

$$
T=\frac{\operatorname{Re} \int_{S_{a}} \mathbf{E} \times \mathbf{H}^{*} \cdot \mathbf{e}_{z} d S}{\operatorname{Re} \int_{S_{a}} \mathbf{E}^{\text {inc }} \times \mathbf{H}^{\text {inc* }} \cdot \mathbf{e}_{z} d S},
$$

which is the ratio of the transmitted power through an aperture to the power incident on the aperture. Re denotes the real part of the complex quantity. Let the incident wave be specified by

$$
Z_{0} \mathbf{H}^{i n c}=\mathbf{e}_{y} e^{-j k z}, \quad \mathbf{E}^{i n c}=\mathbf{e}_{x} e^{-j k z},
$$

where $Z_{0}=\sqrt{\mu_{0} / \epsilon_{0}}$ is the intrinsic impedance of free space, equal to $377 \Omega$.

Since $\mathbf{e}_{z} \times \mathbf{H}$ is real in the $z=0$ plane, we may express the numerator of Eq. (2.79), representing the transmitted power, as

$$
P_{t}=-\operatorname{Re} \int_{S_{a}} \mathbf{J}_{m} \cdot \mathbf{H} d S=R e\langle c, c\rangle,
$$

where $\mathbf{J}_{m}=\mathbf{n} \times \mathbf{E}=\mathbf{E} \times \mathbf{e}_{z}$ is the equivalent magnetic surface current in the aperture and $\langle c, c\rangle$ is the Rumsey's notation for the self-reaction of the correct magnetic 
current radiating in the presence of an electric conducting screen. We approximate $\langle c, c\rangle$ by $\langle a, a\rangle$ with the constraint given by Eq. (2.57) such that

$$
\langle c, c\rangle \approx\langle a, a\rangle=\langle c, a\rangle=\langle a, c\rangle .
$$

The meaning of $\langle c, a\rangle$ is restated as the reaction of the correct field $\mathbf{H}$ on the trial current $\mathbf{J}_{m}^{a}$. The constraint condition is met for our problem because $\mathbf{n} \times \mathbf{H}^{c}=\mathbf{n} \times \mathbf{H}^{\text {inc }}$ in the aperture. A variational formula for $\langle c, c\rangle$, which is stationary with respect to the correct magnetic current $\mathbf{J}_{m}^{c}$, may be written as

$$
<c, c>\approx \frac{\langle c, a\rangle^{2}}{<a, a>}=-\frac{\left(\int_{S_{a}} \mathbf{H}^{i n c} \cdot \mathbf{J}_{m}^{a} d S\right)^{2}}{\int_{S_{a}} \mathbf{H}^{a} \cdot \mathbf{J}_{m}^{a} d S},
$$

where we must emphasize that $\mathbf{H}^{a}$ is the field due to the assumed current $\mathbf{J}_{m}^{a}$.

Thus the variational solution of the transmission coefficient is

$$
T=\frac{-1}{Z_{0} A} \operatorname{Re} \frac{\left(\int_{S_{a}} \mathbf{H}^{i n c} \cdot \mathbf{J}_{m}^{a} d S\right)^{2}}{\int_{S_{a}} \mathbf{H}^{a} \cdot \mathbf{J}_{m}^{a} d S}
$$

where $P_{i}=Z_{0} A$ represents the incident power on the aperture of area $A$.

As a choice of trial fields, Meixner has shown that, at the rim of an aperture, the tangential component of the electric field vanishes as $R^{1 / 2}$ and the normal component increases as $R^{-1 / 2}$ where $R$ measures the distance from the field point to the edge. Bouwkamp has obtained the low-frequency exact solution for the normal incidence on the circular hole of radius a. According to him,

$$
\begin{aligned}
\mathbf{e}_{\rho} \cdot \mathbf{E}(\rho, \phi) & =\frac{2 a^{2}-\rho^{2}}{\left(a^{2}-\rho^{2}\right)^{1 / 2}} \cos \phi, \\
\mathbf{e}_{\phi} \cdot \mathbf{E}(\rho, \phi) & =-2\left(a^{2}-\rho^{2}\right)^{1 / 2} \sin \phi
\end{aligned}
$$

in the hole. Here the origin of the polar coordinate system is at the center of the 
circular hole. Thus we may choose the trial field in the aperture in the form

$$
\begin{aligned}
\mathbf{e}_{\rho} \cdot \mathbf{E}(\rho, \phi) & =\frac{1}{\left(1-\frac{\rho^{2}}{a^{2}}\right)^{1 / 2}} \sum_{0}^{\infty} a_{n}\left(\frac{\rho}{a}\right)^{2 n} \cos \phi, \\
\mathbf{e}_{\phi} \cdot \mathbf{E}(\rho, \phi) & =\left(1-\frac{\rho^{2}}{a^{2}}\right)^{1 / 2} \sum_{0}^{\infty} b_{n}\left(\frac{\rho}{a}\right)^{2 n} \sin \phi,
\end{aligned}
$$

where $a_{n}$ and $b_{n}$ are unknown coefficients to be determined. Huang, Kodis, and Levine [15] have used this trial field to calculate the transmission coefficient and have shown that the agreement between the calculation and measurement is excellent.

As an example of calculational procedure, we adopt here a simpler form of the original trial function used by Levine and Schwinger [21]. With the incident field specified in Eq. (2.80), we may assume a one-component trial field as

$$
\mathbf{n} \times \mathbf{E}^{a}=\mathbf{e}_{x} \sum_{1}^{\infty} a_{n}\left(1-\frac{\rho^{2}}{a^{2}}\right)^{n-1 / 2}=\mathbf{e}_{x} \phi(\rho) .
$$

Substituting this into Eq. (2.84) and using the relation

$$
\mathbf{H}(\mathbf{r})^{a}=-2 j \omega \epsilon \int_{S_{a}} \overline{\mathbf{G}}_{0}\left(\mathbf{r} \mid \mathbf{r}^{\prime}\right) \cdot\left[\mathbf{n} \times \mathbf{E}^{a}\left(\mathbf{r}^{\prime}\right)\right] d S^{\prime}
$$

we obtain

$$
\begin{aligned}
T & =\frac{1}{Z_{0} A} \operatorname{ReI} \\
& =\frac{1}{Z_{0} A} \operatorname{Re} \frac{\left(\int_{S_{a}} \phi(\rho) d s\right)^{2}}{j \omega \epsilon \int_{S_{a}} \int_{S_{a}} \phi(\rho)\left(G_{x x}+G_{y y}\right) \phi\left(\rho^{\prime}\right) d S d S^{\prime}}
\end{aligned}
$$

If we denote

$$
B_{n}=\int_{S_{a}}\left(1-\frac{\rho^{2}}{a^{2}}\right)^{n-1 / 2} d S=\frac{2 \pi a^{2}}{2 n+1}
$$

and

$$
\begin{aligned}
C_{m n} & =\int_{S_{a}} \int_{S_{a}}\left(1-\frac{\rho^{2}}{a^{2}}\right)^{m-1 / 2}\left(G_{x x}+G_{y y}\right)\left(1-\frac{\rho^{2}}{a^{2}}\right)^{n-1 / 2} d S d S^{\prime} \\
& =C_{n m},
\end{aligned}
$$


we may rewrite Eq. (2.89) as

$$
(j \omega \epsilon) I \sum_{m, n=1}^{\infty} a_{m} a_{n} C_{m n}=\left(\sum_{n=1}^{\infty} a_{n} B_{n}\right)^{2} .
$$

The procedure for determining unknown coefficients $a_{n}$ is to differentiate $I$ with respect to each coefficient $a_{\nu}$ and set $\partial I / \partial a_{\nu}=0$. This leads to

$$
(j \omega \epsilon) I \sum_{m} C_{n m} a_{m}=B_{n}\left(\sum_{m} a_{m} B_{m}\right) .
$$

But by definition $I=\sum_{n} a_{n} B_{n}$, so that we have a set of linear algebraic equations for $a_{m}$

$$
\sum_{m=1}^{\infty} C_{n m} a_{m}=\frac{B_{n}}{j \omega \epsilon}
$$

Once $a_{m}$ is determined, the transmission coefficient will be obtained from

$$
T^{(N)}=\frac{1}{Z_{0} A} \operatorname{Re} \sum_{n=1}^{N} I=\frac{1}{Z_{0} A} \operatorname{Re} \sum_{n=1}^{N} a_{n} B_{n},
$$

where $N$ denotes the order of approximation and $R e$ denotes the real part.

As an example, we will work out the first-order approximation. For $N=1, a_{1}$ is simply $j \omega \epsilon a_{1}=B_{1} / C_{11}$. Levine and Schwinger [21] have shown that the coefficients $C_{m n}$ can be expressed as

$$
C_{m n}=\frac{\pi}{2}\left(\frac{2}{k a}\right)^{2} a^{3} \Gamma\left(m+\frac{1}{2}\right) \Gamma\left(n+\frac{1}{2}\right)\left[(m+n-3) F_{m n}(k a)-k a F_{m n}^{\prime}(k a)\right]
$$

where $a$ is the radius of a circular hole and the prime denotes differentiation with respect to the argument. The function $F_{m n}(\alpha)$ has been considered in Ref.[19, 20] and its explicit expression is given there for $m, n=1,2$. Then, with Eq. (2.95), $T^{(1)}$ becomes

$$
T^{(1)}=-\frac{8}{9 \pi} k a \operatorname{Im} \frac{1}{F_{11}(k a)+k a F_{11}^{\prime}(k a)}
$$


which is exactly the same as Eq. (7.16) in Ref. [21] except for the sign because we have used $e^{j \omega t}$ instead of $e^{-i \omega t}$ in Ref. [21]. We also point out the differences between Levine and Schwinger's work and ours in deriving Eq. (2.97). First, we used Rumsey's reaction concept to derive the variational formula. Second, we used M.K.S. units instead of cgs units. Because of these differences, the intermediate results in deriving Eq. (2.97) look different but the final results are of the same form.

The transmission coefficients obtained from the various theories, which are expanded in powers of $k a$, are:

1. Small hole approximation (due to Bethe [5])

$$
T=\frac{64}{27 \pi^{2}}(k a)^{4} .
$$

2. First- and second-order approximations by the variational method using Eq. (2.87) as the trial fields (due to Levine and Schwinger [21])

$$
\begin{aligned}
& T^{(1)}=\frac{64}{27 \pi^{2}}(k a)^{4}\left[1+\frac{27}{25}(k a)^{2}+0.72955(k a)^{4}+\cdots\right], \\
& T^{(2)}=\frac{64}{27 \pi^{2}}(k a)^{4}\left[1+\frac{27}{25}(k a)^{2}+0.74155(k a)^{4}+\cdots\right] .
\end{aligned}
$$

3. Zeroth-order approximation by the variational method using Eqs. (2.85) and (2.86) as the trial fields (due to Levine and Schwinger [21])

$$
T^{(0)}=\frac{64}{27 \pi^{2}}(k a)^{4}\left[1+\frac{22}{25}(k a)^{2}+0.4079(k a)^{4}+\cdots\right] .
$$

4. First-order approximation by the variational method using Eqs. (2.85) and (2.86) as the trial fields (due to Huang, Kodis, and Levine [15])

$$
T^{(1)}=\frac{64}{27 \pi^{2}}(k a)^{4}\left[1+\frac{22}{25}(k a)^{2}+0.3968(k a)^{4}+\cdots\right] .
$$


5. Exact solution (due to Bouwkamp [3])

$$
T=\frac{64}{27 \pi^{2}}(k a)^{4}\left[1+\frac{22}{25}(k a)^{2}+0.3979(k a)^{4}+\cdots\right] \text {. }
$$

(Original work of Bouwkamp is not available to us but the above result is cited in Ref. $[15,21]$.)

We note that Bethe's result is accurate only at low frequencies, namely $k a \ll 1$. The variational method using one of the simplest trial fields shows a good agreement with the exact result, even when such a trial field does not incorporate the proper two-component tangential electric fields in the aperture. Inclusion of two-component effects raises the accuracy up to the relative order $(k a)^{2}$ even in zeroth-order approximation as shown in Eq. (2.101). This demonstrates the importance of choosing correct trial fields. 


\section{Chapter 3}

\section{Longitudinal Impedance of a Hole in an Infinite Screen}

\subsection{Introduction}

In this chapter, we evaluate the longitudinal impedance of a hole in a thin, perfectly conducting plane screen. We have already discussed a similar problem of calculating the transmission coefficient in Chapter 2. Since the analytical technique presented in Chapter 2 is general enough to deal with the calculation of a variety of interesting quantities, it is not necessary to introduce new formalisms for our impedance calculation. After all, calculating the impedance or the transmission coefficient deals with the same diffraction phenomenon. Only the definitions which involve the integration of fields are different.

In Section 3.2, we will estimate the impedance in the low frequency range using Bethe's [5] small hole approximation. If we can expand in powers of frequency, the low frequency solution will represent the first term in the series.

In Section 3.3, we will obtain the variational solution of impedance with different trial fields, showing the main result of this chapter. 


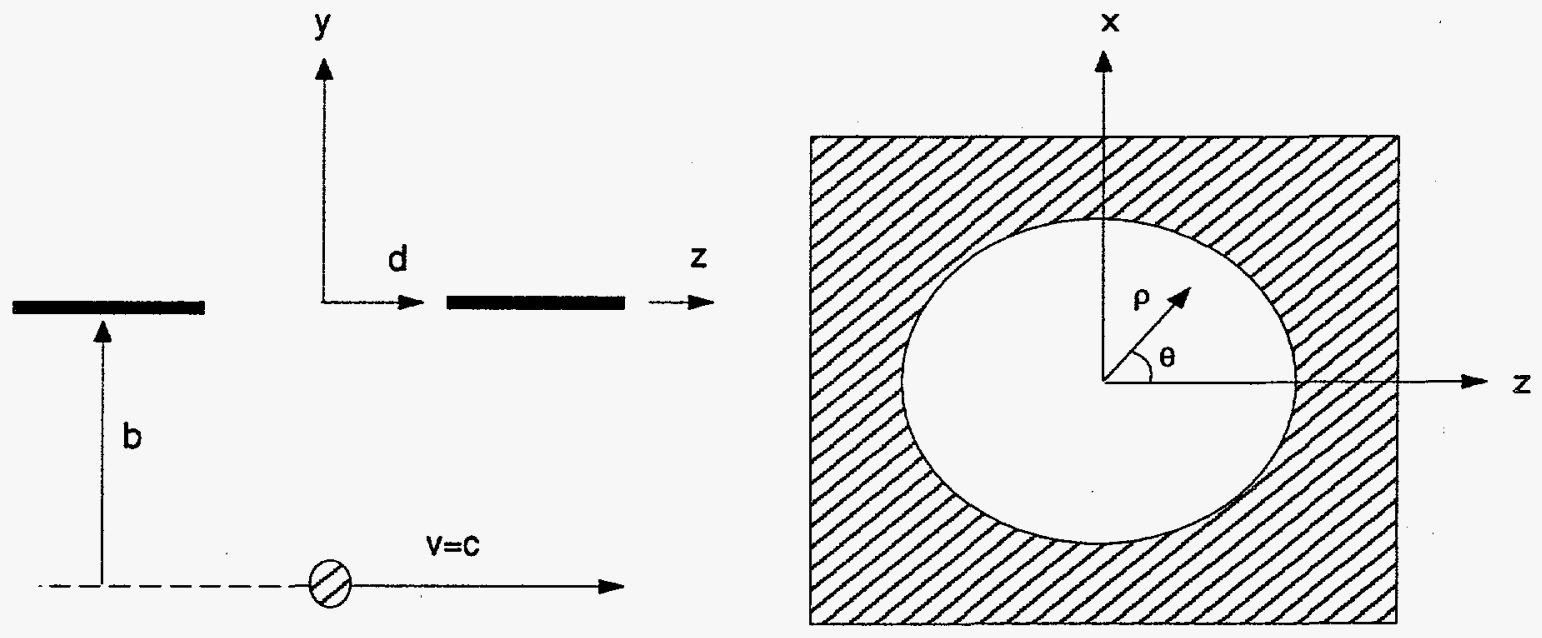

Figure 3.1: Infinite flat screen with a hole.

\subsection{Low Frequency Solution}

The geometry of our problem is shown in Fig. 3.1 where a charge is moving in the z-direction with velocity close to the speed of light. The distance between the plane screen and the beam path is $b$ and the origin of the coordinate is at the center of the hole with radius $d$. The local cylindrical coordinate system $(\rho, \theta, y)$ is also shown.

Denoting $\mathbf{E}_{1}, \mathbf{H}_{1}$ as the fields without the hole and $\mathbf{E}_{2}, \mathbf{H}_{2}$ as the fields with the hole, we can express the longitudinal coupling impedance defined by Eq. (1.14) as

$$
\left|I_{0}\right|^{2} Z(k)=\int_{\text {hole }}\left(\mathbf{n} \times \mathbf{E}_{2}\right) \cdot \mathbf{H}_{\mathbf{1}}^{*} d S,
$$

where $\mathbf{J}_{m}=\mathbf{n} \times \mathbf{E}_{2}$ is the magnetic current induced in the hole, which is not known until we solve the problem. In the low frequency approximation, however, we may use Bethe's solution for $\mathbf{n} \times \mathbf{E}$ as follows.

Assuming a small hole, namely $k d=2 \pi d / \lambda \ll 1$, Bethe obtained the solution for 
the magnetic current in the hole [5]

$$
\mathbf{n} \times \mathbf{E}=-\frac{\rho}{\pi \sqrt{d^{2}-\rho^{2}}} \mathbf{e}_{\rho} \times \mathbf{E}_{0}+j \frac{2 k Z_{0}}{\pi} \sqrt{d^{2}-\rho^{2}} \mathbf{H}_{0}=\mathbf{J}_{m, E}+\mathbf{J}_{m, H},
$$

where $\mathbf{E}_{0}$ and $\mathbf{H}_{0}$ are the field evaluated at the center of the hole in the absence of the hole, and $\mathbf{J}_{m, E}$ and $\mathbf{J}_{m, H}$ denote the magnetic current induced in the hole due to the incident electric and magnetic field, respectively.

The magnetic field from the unit source current can be obtained using the image principle, resulting in

$$
\begin{aligned}
& H_{x}=\frac{I_{0}}{2 \pi}\left[\frac{(y-b)}{x^{2}+(y-b)^{2}}-\frac{(y+b)}{x^{2}+(y+b)^{2}}\right] e^{-j k z}, \\
& H_{z}=\frac{I_{0}}{2 \pi}\left[\frac{x}{x^{2}+(y+b)^{2}}-\frac{x}{x^{2}+(y-b)^{2}}\right] e^{-j k z},
\end{aligned}
$$

where the coordinate system in Fig. 3.1 is used. In the plane of the hole, it becomes

$$
H_{x}=-\frac{I_{0}}{\pi} \frac{b}{x^{2}+b^{2}} e^{-j k z}, H_{z}=0 .
$$

Assuming a small hole in which the field is uniform but the phase is varying, we may rewrite the source field as

$$
\mathbf{H}_{1}=\mathbf{H}_{0}-j k z \mathbf{H}_{0}+\mathcal{O}\left(k^{2}\right), \text { where } \mathbf{H}_{0}=-\frac{I_{0}}{\pi b} \mathbf{e}_{x} .
$$

Then, the longitudinal coupling impedance becomes

$$
\begin{aligned}
\left|I_{0}\right|^{2} Z_{H}(k) & =\int_{\text {hole }} \mathbf{J}_{m, H} \cdot \mathbf{H}_{1}^{*} d S=j \frac{4 Z_{0} d^{3} H_{0}^{2}}{3} k, \\
\left|I_{0}\right|^{2} Z_{E}(k) & =\int_{\text {hole }} \mathbf{J}_{m, E} \cdot \mathbf{H}_{1}^{*} d S=-j \frac{2 Z_{0} d^{3} H_{0}^{2}}{3} k, \\
\left|I_{0}\right|^{2} Z(k) & =Z_{H}(k)+Z_{E}(k)=j \frac{2 Z_{0} d^{3} H_{0}^{2}}{3} k
\end{aligned}
$$


which results in $Z(k)=\left(2 Z_{0} d^{3} / 3 \pi^{2} b^{2}\right) k$. We note that $Z_{H}(k)$ due to $\mathbf{J}_{m, H}$ is a factor of two larger than $Z(k)$, and $Z_{E}(k)$ due to $\mathbf{J}_{m, E}$ is the negative of $Z(k)$. We also observe that the current $I_{0}$ does not appear in the final expression of impedance. Thus we keep $I_{0}$ in the expression only for bookkeeping purposes, setting $I_{0}=1$ in the final formula. By doing so, we may simplify the expression in the later sections.

Although Eq. (3.8) is valid only for low frequencies, it is quite general. If we consider a charge traveling inside a cylindrical beam pipe of radius $b$ with a hole of radius $d$, the longitudinal coupling impedance becomes, with $H_{0}=\frac{I_{0}}{2 \pi b}$ in Eq. (3.8),

$$
Z(k)=j \frac{Z_{0} d^{3}}{6 \pi^{2} b^{2}} k
$$

which is exactly the same as Kurennoy's [18] and Gluckstern's results [11].

Thus, in low frequency approximation, we can easily obtain the longitudinal coupling impedance once we know the source field which is specific to the geometry surrounding a traveling charge.

\subsection{Variational Solutions}

In this section, we try to calculate the longitudinal coupling impedance of a hole in an infinite screen using a variational method. We begin by defining an "impedance functional" which is stationary with respect to the unknown quantity (magnetic current density in the hole). Such a functional may not be the same as the quantity of interest, the longitudinal coupling impedance, but it will be shown later that the two quantities are related. Thus, from the impedance functional, we can obtain the expression for the longitudinal coupling impedance. Various trial fields are used to evaluate the impedance functional and the results are presented. 


\subsubsection{Derivation of Impedance Functional}

The geometry of the problem is shown in Fig. 3.1. If we denote the fields without the hole in the screen as $\mathbf{E}_{1}$ and $\mathbf{H}_{1}$, and the fields with the hole in the screen as $\mathbf{E}_{2}$ and $\mathbf{H}_{2}, \mathbf{E}_{1,2}$ and $\mathbf{H}_{1,2}$ satisfy Maxwell's equations in the form

$$
\nabla \times \mathbf{E}_{1,2}=-j \omega \mu \mathbf{H}_{1,2}, \quad \nabla \times \mathbf{H}_{1,2}=j \omega \epsilon \mathbf{E}_{1,2}+\mathbf{J},
$$

where $\mathbf{J}=\mathbf{e}_{\mathbf{z}} \delta(y+d) \delta(x) e^{-j k z}$ is the current density of the driving beam with unit amplitude and $k=\omega \sqrt{\epsilon_{0} \mu_{0}}$ is the free-space wave number.

Expanding the quantity $\nabla \cdot\left(\mathbf{E}_{1} \times \mathbf{H}_{2}-\mathbf{E}_{2} \times \mathbf{H}_{1}\right)$, we get the identity

$$
\nabla \cdot\left(\mathbf{E}_{1} \times \mathbf{H}_{2}-\mathbf{E}_{2} \times \mathbf{H}_{1}\right)=\mathbf{J} \cdot\left(\mathbf{E}_{2}-\mathbf{E}_{1}\right) .
$$

Taking the integral of both sides, we have

$$
\int\left(\mathbf{E}_{1} \times \mathbf{H}_{2}-\mathbf{E}_{2} \times \mathbf{H}_{1}\right) \cdot \mathbf{n} d S=\int \mathbf{J} \cdot\left(\mathbf{E}_{2}-\mathbf{E}_{1}\right) d V .
$$

We define the impedance functional $\mathcal{Z}$ as

$$
\mathcal{Z}=-\int \mathbf{J} \cdot\left(\mathbf{E}_{2}-\mathbf{E}_{1}\right) d V
$$

In the above definition, as we subtracted the contribution from the source field, the entire contribution is from the scattered field which satisfies the homogeneous Maxwell's equations. We note that if the electric field is real, the longitudinal impedance is the complex conjugate of the impedance functional, $Z(k)=\mathcal{Z}^{*}(k)$.

If the integrating surface is chosen to coincide with the plane of the screen where $\mathbf{E}_{1}$ satisfies the boundary condition $\mathbf{n} \times \mathbf{E}_{1}=0, \mathcal{Z}$ reduces to

$$
\mathcal{Z}=\int \mathbf{H}_{1} \cdot\left(\mathbf{n} \times \mathbf{E}_{2}\right) d S,
$$


which we want to evaluate. We also recall that the longitudinal impedance is

$$
Z(k)=\int \mathbf{H}_{\mathbf{1}}^{*} \cdot\left(\mathbf{n} \times \mathbf{E}_{\mathbf{2}}\right) d S .
$$

For the sake of clarity, we change the notation as

$$
\begin{array}{ll}
\mathbf{E}_{1}=\mathbf{E}^{i}, & \mathbf{H}_{1}=\mathbf{H}^{i}, \\
\mathbf{E}_{2}=\mathbf{E}^{i}+\mathbf{E}^{s}, & \mathbf{H}_{2}=\mathbf{H}^{i}+\mathbf{H}^{s},
\end{array}
$$

where superscript $i$ denotes the "incident" or "source" field of the driving beam without the hole, and superscript $s$ denotes the "scattered" field from the hole. The hole is acting as "scattering object" or "obstacle"; otherwise the media are empty space surrounded or separated by the perfectly conducting material.

In the new notation, we may write $\mathcal{Z}$ as

$$
\mathcal{Z}=\int \mathbf{H}^{i} \cdot\left(\mathbf{n} \times \mathbf{E}^{s}\right) d S,
$$

where $\mathbf{n} \times \mathbf{E}^{i}=0$ in the aperture is used. With $\mathbf{J}_{m}=\mathbf{n} \times \mathbf{E}^{s}, \mathcal{Z}$ becomes

$$
\mathcal{Z}=\int \mathbf{H}^{i} \cdot \mathbf{J}_{m} d S
$$

But, by definition, $\mathcal{Z}=-\int \mathbf{J} \cdot \mathbf{E}^{s} d S$ so that this satisfies Lorentz's reciprocity theorem. Using Rumsey's notation, we may express $\mathcal{Z}$ in a compact form, $\mathcal{Z}=-\langle i, s\rangle$ which is often called the "echo" of a scattering object, an important quantity in the development of radar technology. We can state that our impedance functional is the mutual reaction between the source current and the magnetic current induced in the aperture. Using known boundary conditions which the tangential magnetic field must satisfy in the aperture, $H_{t}^{i}=-2 H_{t}^{s}$, we rewrite

$$
\mathcal{Z}=-2 \int \mathbf{H}^{s} \cdot \mathbf{J}_{m} d S=2\langle c, c\rangle
$$


where $\langle c, c\rangle$ stands for the self-reaction of the "correct" magnetic current induced in the aperture by the source.

Now we can write the variational expression for $\mathcal{Z}$ as

$$
\mathcal{Z}=2\left\langle c, c>\approx 2 \frac{\left\langle c, a>^{2}\right.}{<a, a>}=\frac{-1}{2} \frac{\left[\int_{S_{a}} \mathbf{H}^{i} \cdot\left(\mathbf{n} \times \mathbf{E}^{a}\right) d S\right]^{2}}{\int_{S_{a}} \mathbf{H}^{a} \cdot\left(\mathbf{n} \times \mathbf{E}^{a}\right) d S} .\right.
$$

Using dyadic Green's function, we calculate $\mathbf{H}^{a}$ from

$$
\mathbf{H}^{a}(\mathbf{r})=-2 j \omega \epsilon \int_{S_{a}} \overline{\mathbf{G}}_{\mathbf{0}}\left(\mathbf{r} \mid \mathbf{r}^{\prime}\right) \cdot\left[\mathbf{n} \times \mathbf{E}^{a}\left(\mathbf{r}^{\prime}\right)\right] d S^{\prime} .
$$

Thus, Eq. (3.19) may be rewritten as

$$
\mathcal{Z}=\frac{1}{4 j \omega \epsilon} \frac{\left[\int_{S_{a}} \mathbf{H}^{i} \cdot\left(\mathbf{n} \times \mathbf{E}^{a}\right) d S\right]^{2}}{\int_{S_{a}} \int_{S_{a}}\left[\mathbf{n} \times \mathbf{E}^{a}(\mathbf{r})\right] \cdot \overline{\mathbf{G}}_{0}\left(\mathbf{r} \mid \mathbf{r}^{\prime}\right) \cdot\left[\mathbf{n} \times \mathbf{E}^{a}\left(\mathbf{r}^{\prime}\right)\right] d S d S^{\prime}}
$$

which is a homogeneous equation in the sense that the result does not depend on the amplitude of the assumed electric field $\mathbf{E}^{a}$. In fact this is a general expression for the impedance functional of an aperture in a conducting plane as long as the plane is the symmetry plane separating two regions, namely, an infinite plane or coupled waveguide structure. Details of the calculation depend on the shape of the aperture and the assumed tangential electric field in the aperture. In the next section, we present a closed form solution for a hole in an infinite screen.

\subsubsection{The First Variational Solution}

In the previous section, we derived the impedance functional $\mathcal{Z}$ which is stationary with respect to the correct tangential electric field in the aperture. In order to evaluate it, we need to assume a trial function for $\mathbf{E}^{a}$.

We assume a one-component trial field based on Bethe's solution [5] as

$$
\mathbf{n} \times \mathbf{E}=\mathbf{e}_{x} \sum_{n=1}^{n=\infty} a_{n}\left(1-\frac{\rho^{2}}{d^{2}}\right)^{n-\frac{1}{2}}=\mathbf{e}_{x} \phi(\rho),
$$


where we dropped the superscript $a$. We note that the unknown coefficient $a_{n}$ is dependent on the frequency. The impedance functional is

$$
\mathcal{Z}=\frac{1}{4 j \omega \epsilon} \frac{\left[\int_{S_{a}} H_{x}^{i}(\rho, \theta) \phi(\rho) d S\right]^{2}}{\int_{S_{a}} \int_{S_{a}} \phi(\rho) G_{x x}\left(\rho, \theta \mid \rho^{\prime}, \theta^{\prime}\right) \phi\left(\rho^{\prime}\right) d S d S^{\prime}}
$$

We transform the equation into a more symmetrical form utilizing the symmetry of a circle. For instance, if the beam is moving in the $x$ direction, the expression for $\mathcal{Z}$ will be in the same functional form as Eq. (3.23) except that the subscript $x$ denoting the $x$ component of the field or dyadic Green's function is replaced by $z$. Thus, we have

$$
\mathcal{Z}=\frac{1}{2 j \omega \epsilon} \frac{\left[\int_{S_{a}} H_{x}^{i}(\rho, \theta) \phi(\rho) d S\right]^{2}}{\int_{S_{a}} \int_{S_{a}} \phi(\rho)\left(G_{x x}+G_{z z}\right) \phi\left(\rho^{\prime}\right) d S d S^{\prime}}
$$

which is later shown to be much simpler to evaluate.

If we define

$$
B_{n}=\int H_{x}^{i}(\rho, \theta)\left(1-\frac{\rho^{2}}{d^{2}}\right)^{n-\frac{1}{2}} d S
$$

and

$$
C_{m n}=\iint\left(1-\frac{\rho^{2}}{d^{2}}\right)^{m-\frac{1}{2}}\left(G_{x x}+G_{z z}\right)\left(1-\frac{\rho^{2}}{d^{2}}\right)^{n-1 / 2} d S d S^{\prime}=C_{n m}
$$

$\mathcal{Z}$ becomes

$$
\mathcal{Z}=\frac{1}{2 j \omega \epsilon} \frac{\left(\sum_{n=1} a_{n} B_{n}\right)^{2}}{\sum_{n, m=1} a_{m} a_{n} C_{m n}}
$$

or

$$
(2 j \omega \epsilon) \mathcal{Z} \sum_{n, m=1} a_{m} a_{n} C_{m n}=\left(\sum_{n} a_{n} B_{n}\right)^{2} .
$$

Differentiation with respect to $a_{\nu}$, and utilization of the stationary property of $\mathcal{Z}$, namely $\partial \mathcal{Z} / \partial a_{\nu}=0$, yield

$$
(2 j \omega \epsilon) \sum_{m} a_{m} C_{m n}=B_{n}
$$


where we used the identity $\mathcal{Z}=\sum_{n} a_{n} B_{n}$. Once the frequency-dependent coefficient $a_{n}$ is determined by solving the linear algebraic equation, Eq. (3.29), the desired impedance functional can be obtained from

$$
\mathcal{Z}^{(N)}=\sum_{n=1}^{N} a_{n} B_{n}
$$

where $N$ is the order of approximation. Since the longitudinal coupling impedance is defined by $Z(k)=\sum_{n=1}^{N} a_{n} B_{n}^{*}$ and $B_{n}=B_{n}^{*}$, which will be shown later, the impedance functional is the same as the longitudinal coupling impedance.

The lowest order approximation, $N=1$, results in

$$
\begin{aligned}
a_{1} & =\frac{B_{1}}{2 j \omega \epsilon C_{11}}, \\
\mathcal{Z}^{(1)} & =-j \frac{Z_{0}}{2 k}\left(\frac{B_{1}^{2}}{C_{11}}\right),
\end{aligned}
$$

where $k=\omega \sqrt{\epsilon_{0} \mu_{0}}$ is the free-space wave number and $Z_{0}=377 \Omega$.

With $\mathrm{N}=2$, we find

$$
\begin{aligned}
a_{1} & =-j \frac{Z_{0}}{2 k}\left[\frac{B_{1} C_{22}-B_{2} C_{12}}{C_{11} C_{22}-C_{12}^{2}}\right], \\
a_{2} & =-j \frac{Z_{0}}{2 k}\left[\frac{B_{2} C_{11}-B_{1} C_{12}}{C_{11} C_{22}-C_{12}^{2}}\right], \\
\mathcal{Z}^{(2)} & =-j \frac{Z_{0}}{2 k}\left[\frac{B_{1}^{2}}{C_{11}}+\frac{\left(B_{2} C_{11}-B_{1} C_{12}\right)^{2}}{C_{11}\left(C_{11} C_{22}-C_{12}^{2}\right)}\right] \\
& =\mathcal{Z}^{(1)}-j \frac{Z_{0}}{2 k} \frac{\left(B_{2} C_{11}-B_{1} C_{12}\right)^{2}}{C_{11}\left(C_{11} C_{22}-C_{12}^{2}\right)},
\end{aligned}
$$

which shows the successive approximation as the order of approximation is increased.

We turn our attention to finding an explicit expression of the quantities $B_{n}$ and $C_{m n}$ in order to complete the approximate calculation of the impedance. Since the most convenient coordinate system to describe the hole in the flat plane is the cylindrical coordinate system, we express the component of dyadic Green's function in 
that coordinate system and then carry out the necessary integration to obtain $B_{n}$ and $C_{m n}$.

The free-space Green's function satisfying the wave equation

$$
\left(\nabla^{2}+k^{2}\right) G\left(\mathbf{r}, \mathbf{r}^{\prime}\right)=-\delta\left(\mathbf{r}-\mathbf{r}^{\prime}\right)
$$

is $G=e^{-j k R} / 4 \pi R$, where $R=\left|\mathbf{r}-\mathbf{r}^{\prime}\right|$. Its integral representation is

$$
\begin{aligned}
G & =\frac{e^{-j k R}}{4 \pi R} \\
& =\frac{1}{8 \pi^{3}} \int d q_{x} e^{-j q_{x}\left(x-x^{\prime}\right)} \int d q_{z} e^{-j q_{z}\left(z-z^{\prime}\right)} \int d q_{y} \frac{e^{-j q_{y}\left(y-y^{\prime}\right)}}{q_{x}^{2}+q_{z}^{2}+q_{y}^{2}-k^{2}}
\end{aligned}
$$

where the Cartesian coordinate system is defined in Fig. 3.1. With the help of a contour which will result in the outgoing wave satisfying the radiation condition at infinity, Eq. (3.33) becomes

$$
G=\frac{1}{8 \pi^{2}} \iint d q_{x} d q_{z} \frac{e^{-j\left[q_{x}\left(x-x^{\prime}\right)+q_{z}\left(z-z^{\prime}\right)+\sqrt{k^{2}-q_{x}^{2}-q_{z}^{2}}\left|y-y^{\prime}\right|\right]}}{\sqrt{q_{x}^{2}+q_{z}^{2}-k^{2}}} .
$$

For the convergence of the above integral, we require that $I m \sqrt{k^{2}-q_{x}^{2}-q_{z}^{2}}$ is negative which also implies $I m \sqrt{q_{x}^{2}+q_{z}^{2}-k^{2}}$ positive.

We are now ready to evaluate the individual component of dyadic Green's function. Specifically, we will work out $G_{x x}$ and $G_{z z}$.

$$
\begin{aligned}
G_{z z} & =\left(1+\frac{1}{k^{2}} \frac{\partial^{2}}{\partial z^{2}}\right) G \\
& =\frac{1}{8 \pi^{2}} \iint d q_{x} d q_{z} \frac{e^{-j\left[q_{x}\left(x-x^{\prime}\right)+q_{z}\left(z-z^{\prime}\right)+\sqrt{k^{2}-q_{x}^{2}-q_{z}^{2} \mid} y-y^{\prime} \mid\right]}}{\sqrt{q_{x}^{2}+q_{z}^{2}-k^{2}}}\left(1-\frac{q_{z}^{2}}{k^{2}}\right) .
\end{aligned}
$$

Introducing a set of angle variables,

$$
\begin{aligned}
& \mathbf{q}=\underset{z}{\mathbf{e}_{z}}+q_{x} \mathbf{e}_{x}=(q \cos u) \mathbf{e}_{z}+(q \sin u) \mathbf{e}_{x}, \\
& \mathbf{R}=\left(z-z^{\prime}\right) \mathbf{e}_{z}+\left(x-x^{\prime}\right) \mathbf{e}_{x}=(R \cos v) \mathbf{e}_{z}+(R \sin v) \mathbf{e}_{x}
\end{aligned}
$$


we obtain

$$
\begin{aligned}
G_{z z}= & \frac{1}{8 \pi^{2}} \int_{0}^{\infty} \frac{q d q}{\sqrt{q^{2}-k^{2}}} e^{-j \sqrt{k^{2}-q^{2}}\left|y-y^{\prime}\right|} \int_{0}^{2 \pi} e^{-j q R \cos (v-u)} d u \\
& -\frac{1}{8 \pi^{2}} \int_{0}^{\infty} \frac{q d q}{\sqrt{q^{2}-k^{2}}}\left(\frac{q^{2}}{k^{2}}\right) e^{-j \sqrt{k^{2}-q^{2}}\left|y-y^{\prime}\right|} \int_{0}^{2 \pi} e^{-j q R \cos (v-u)} \cos ^{2} u d u .
\end{aligned}
$$

Using an integral formula

$$
\int_{0}^{\pi} e^{ \pm j z \cos \theta} \sin ^{2 \nu} \theta d \theta=\frac{\Gamma\left(\nu+\frac{1}{2}\right) \Gamma\left(\frac{1}{2}\right)}{(z / 2)^{\nu}} J_{\nu}(z)
$$

we find

$$
G_{z z}=\frac{1}{4 \pi} \int_{0}^{\infty} \frac{q d q}{\sqrt{q^{2}-k^{2}}}\left[J_{0}(q R)-\frac{q^{2}}{2 k^{2}}\left(J_{0}(q R)-\cos 2 v J_{2}(q R)\right)\right] e^{-j \sqrt{k^{2}-q^{2}}\left|y-y^{\prime}\right|}
$$

A similar expression for $G_{x x}$ is

$$
G_{x x}=\frac{1}{4 \pi} \int_{0}^{\infty} \frac{q d q}{\sqrt{q^{2}-k^{2}}}\left[J_{0}(q R)-\frac{q^{2}}{2 k^{2}}\left(J_{0}(q R)+\cos 2 v J_{2}(q R)\right)\right] e^{-j \sqrt{k^{2}-q^{2}}\left|y-y^{\prime}\right|}
$$

On the aperture plane $y=y^{\prime}=0$, the desired expression for $G_{x x}+G_{z z}$ takes a simpler form,

$$
\begin{aligned}
G_{x x}+G_{z z} & =\left[\left(1+\frac{1}{k^{2}} \frac{\partial^{2}}{\partial x^{2}}\right)+\left(1+\frac{1}{k^{2}} \frac{\partial^{2}}{\partial z^{2}}\right)\right] G \\
& =\frac{1}{4 \pi} \int_{0}^{\infty} q d q\left[\left(q^{2}-k^{2}\right)^{-1 / 2}-\frac{1}{k^{2}}\left(q^{2}-k^{2}\right)^{1 / 2}\right] J_{0}(q R) .
\end{aligned}
$$

Using a Bessel function addition theorem,

$$
\begin{aligned}
J_{0}(q R) & =J_{0}\left(q\left(\rho^{2}+\rho^{\prime 2}-2 \rho \rho^{\prime} \cos \left(\theta-\theta^{\prime}\right)\right)^{1 / 2}\right) \\
& =\sum_{0}^{\infty}\left(2-\delta_{0 n}\right) J_{n}(q \rho) J_{n}\left(q \rho^{\prime}\right) \cos n\left(\theta-\theta^{\prime}\right)
\end{aligned}
$$


where $\delta_{0 n}=1$ if $n=0$ and $\delta_{0 n}=0$ if $n \neq 0$, we further transform $G_{x x}+G_{z z}$ as

$$
\begin{aligned}
G_{x x}+G_{z z}= & \frac{1}{4 \pi} \sum_{0}^{\infty}\left(2-\delta_{0 n}\right) \cos n\left(\theta-\theta^{\prime}\right) \\
& \int_{0}^{\infty} q d q\left[\left(q^{2}-k^{2}\right)^{-1 / 2}-\frac{1}{k^{2}}\left(q^{2}-k^{2}\right)^{1 / 2}\right] J_{n}(q \rho) J_{n}\left(q \rho^{\prime}\right) .
\end{aligned}
$$

Substituting the above expression into Eq. (3.26), we have

$$
\begin{aligned}
C_{m n} & =\pi \int_{0}^{\infty}\left[\left(q^{2}-k^{2}\right)^{-1 / 2}-\frac{1}{k^{2}}\left(q^{2}-k^{2}\right)^{1 / 2}\right] \\
\cdot & \int_{0}^{d} \rho\left(1-\frac{\rho^{2}}{d^{2}}\right)^{m-\frac{1}{2}} J_{0}(q \rho) d \rho \int_{0}^{d} \rho^{\prime}\left(1-\frac{\rho^{2}}{d^{2}}\right)^{n-\frac{1}{2}} J_{0}\left(q \rho^{\prime}\right) d \rho^{\prime}
\end{aligned}
$$

which is to be evaluated.

With the change of variable $\rho=d \sin \varphi$, the first integral in Eq. (3.40) becomes

$$
\begin{aligned}
\int_{0}^{d} \rho\left(1-\frac{\rho^{2}}{d^{2}}\right)^{m-\frac{1}{2}} J_{0}(q \rho) d \rho & =d^{2} \int_{0}^{\frac{\pi}{2}} J_{0}(q d \sin \varphi) \sin \varphi \cos ^{2 m} \varphi d \varphi \\
& =d^{2} \frac{2^{m-\frac{1}{2}} \Gamma\left(m+\frac{1}{2}\right)}{(q d)^{m+\frac{1}{2}}} J_{m+\frac{1}{2}}(q d)
\end{aligned}
$$

where, in deriving the final result, we used Sonine's first finite integral formula ${ }^{1}$,

$$
\int_{0}^{\frac{\pi}{2}} J_{\mu}(z \sin \theta) \sin ^{\mu+1} \theta \cos ^{2 \nu+1} \theta d \theta=\frac{2^{\nu} \Gamma(\nu+1)}{z^{\nu+1}} J_{\mu+\nu+1}(z)
$$

which is valid when both $\operatorname{Re}(\mu)$ and $\operatorname{Re}(\nu)$ exceed -1 .

Substituting the above intermediate result into Eq. (3.40) and introducing a new variable $v=q / k$, we rewrite $C_{m n}$ as

$$
\begin{aligned}
C_{m n}= & \frac{\pi}{2}\left(\frac{2}{k d}\right)^{m+n} d^{3} \Gamma\left(m+\frac{1}{2}\right) \Gamma\left(n+\frac{1}{2}\right) \\
& \int_{0}^{\infty}\left[\left(v^{2}-1\right)^{-1 / 2}-\left(v^{2}-1\right)^{1 / 2}\right] v^{-(m+n)} J_{m+\frac{1}{2}}(k d v) J_{n+\frac{1}{2}}(k d v) d v
\end{aligned}
$$

\footnotetext{
${ }^{1}$ See reference $[30]$, p. 373 .
} 
If we define $F_{m n}(\alpha)$ as

$$
F_{m n}(\alpha)=\int_{0}^{\infty}\left(v^{2}-1\right)^{1 / 2} v^{-(m+n)} J_{m+\frac{1}{2}}(\alpha v) J_{n+\frac{1}{2}}(\alpha v) d v
$$

it can be shown that

$$
C_{m n}=\frac{\pi}{2}\left(\frac{2}{k d}\right)^{m+n} d^{3} \Gamma\left(m+\frac{1}{2}\right) \Gamma\left(n+\frac{1}{2}\right)\left[(m+n-3) F_{m n}(k d)-k d F_{m n}^{\prime}(k d)\right],
$$

where the prime stands for differentiation with respect to the argument. With the substitution of the above into Eq. (3.31), we obtain

$$
\mathcal{Z}^{(1)}=j \frac{Z_{0}}{\pi^{2} d^{2}}(k d) \frac{B_{1}^{2}}{F_{11}(k d)+k d F_{11}^{\prime}(k d)} .
$$

In order to evaluate the function $F_{m n}(\alpha)$, we divide the integration range into two parts,

$$
F_{m n}(\alpha)=R_{m n}(\alpha)+j I_{m n}(\alpha)
$$

where

$$
\begin{aligned}
I_{m n}(\alpha) & =\int_{0}^{1}\left(1-v^{2}\right)^{1 / 2} v^{-(m+n)} J_{m+\frac{1}{2}}(\alpha v) J_{n+\frac{1}{2}}(\alpha v) d v \\
R_{m n}(\alpha) & =\int_{1}^{\infty}\left(v^{2}-1\right)^{1 / 2} v^{-(m+n)} J_{m+\frac{1}{2}}(\alpha v) J_{n+\frac{1}{2}}(\alpha v) d v
\end{aligned}
$$

For the first few values of $m$ and $n$, Levine and Schwinger [19] found

$$
\begin{aligned}
& I_{11}(\alpha)=\frac{\alpha}{2 \pi}-\frac{1}{4 \pi \alpha}+\frac{1}{8 \alpha^{2}} S_{0}(2 \alpha)-\frac{1}{16 \alpha^{3}} \int_{0}^{2 \alpha} S_{0}(t) d t-\frac{1}{4 \alpha} \int_{0}^{2 \alpha} \frac{S_{1}(t)}{t} d t \\
& R_{11}(\alpha)=\frac{1}{4 \alpha}\left(1+\frac{1}{4 \alpha^{2}}\right) \int_{0}^{2 \alpha} J_{0}(t) d t-\frac{1}{8 \alpha^{2}} J_{0}(2 \alpha)-\frac{1}{4 \alpha} J_{1}(2 \alpha)
\end{aligned}
$$

where $S_{0}, S_{1}$ and $J_{0}, J_{1}$ denote the zeroth- and first-order Struve and Bessel functions, respectively. The similarity between the two functions may be illustrated by 
comparing the integral representation of the two functions,

$$
\begin{aligned}
S_{\nu}(z) & =\frac{2(z / 2)^{\nu}}{\Gamma\left(\nu+\frac{1}{2}\right) \Gamma\left(\frac{1}{2}\right)} \int_{0}^{1}\left(1-t^{2}\right)^{\nu-\frac{1}{2}} \sin z t d t \\
J_{\nu}(z) & =\frac{2(z / 2)^{\nu}}{\Gamma\left(\nu+\frac{1}{2}\right) \Gamma\left(\frac{1}{2}\right)} \int_{0}^{1}\left(1-t^{2}\right)^{\nu-\frac{1}{2}} \cos z t d t .
\end{aligned}
$$

We now need to calculate $B_{n}$ in order to obtain the complete expression for the impedance. In the plane of the hole, $y=0$, the source fields become

$$
H_{x}=-\frac{I_{0}}{\pi} \frac{b}{x^{2}+b^{2}} e^{-j k z}, H_{z}=0
$$

Thus,

$$
\begin{aligned}
B_{n} & =\int \mathbf{H}^{i} \cdot(\mathbf{E} \times \mathbf{n}) d S \\
& =-\frac{b}{\pi} \int_{S_{a}}\left(1-\frac{\rho^{2}}{d^{2}}\right)^{n-\frac{1}{2}} \frac{e^{-j k z}}{x^{2}+b^{2}} d S .
\end{aligned}
$$

Assuming that the source current is far from the hole, i.e., $x / b<<1$, and assuming that the field is static, $B_{n}$ becomes

$$
B_{n}=\frac{2 \pi d^{2}}{(2 n+1)} H_{0}=-\frac{2 d^{2}}{(2 n+1) b}
$$

In fact this is the approximation on which Bethe's small hole theory is based. Later, we will factor this quantity out of a whole expression leaving the effect of finite size of the hole and the effect of phase variation along the hole in the compact form. We will call this a form factor.

For general cases, introducing the change of the variables $z=\rho \cos \theta$ and $x=$ $\rho \sin \theta$ and denoting $w=\rho / b$, we find from Eq. (3.51)

$$
B_{n}=2 H_{0} \int_{0}^{d}\left(1-\frac{\rho^{2}}{d^{2}}\right)^{n-\frac{1}{2}} \rho d \rho \int_{0}^{\pi} \frac{e^{-j k b w \cos \theta}}{w^{2} \sin ^{2} \theta+1} d \theta
$$


We integrate the second term to obtain

$$
\begin{aligned}
\int_{0}^{\pi} \frac{e^{-j k b w \cos \theta}}{w^{2} \sin ^{2} \theta+1} d \theta & =\sum_{m=0}^{\infty}(-1)^{m} w^{2 m} \int_{0}^{\pi} e^{-j k b w \cos \theta} \sin ^{2 m} \theta d \theta \\
& =\sum_{m=0}^{\infty}(-1)^{m} \frac{2^{m} \rho^{m}}{b^{2 m} k^{m}} \Gamma\left(m+\frac{1}{2}\right) \Gamma\left(\frac{1}{2}\right) J_{m}(k \rho) .
\end{aligned}
$$

Substituting the above into Eq. (3.52) and using

$$
\begin{aligned}
\int_{0}^{d}\left(1-\frac{\rho^{2}}{d^{2}}\right)^{n-\frac{1}{2}} \rho^{m+1} J_{m}(k \rho) d \rho & =d^{m+2} \int_{0}^{\frac{\pi}{2}} J_{m}(k d \sin \theta) \cos ^{2 n} \theta \sin ^{m+1} \theta d \theta \\
& =d^{m+2} \frac{2^{n-\frac{1}{2}} \Gamma\left(n+\frac{1}{2}\right)}{(k d)^{n+\frac{1}{2}}} J_{m+n+\frac{1}{2}}(k d)
\end{aligned}
$$

we may rewrite $B_{n}$ as

$$
\begin{aligned}
B_{n}= & \frac{2 \pi d^{2}}{2 n+1} H_{0} F F_{n}\left(\frac{d}{b}, k d\right) \\
F F_{n}\left(\frac{d}{b}, k d\right)= & \sqrt{\frac{\pi}{2}}(2 n+1) ! ! \\
& {\left[\frac{J_{n+\frac{1}{2}}(k d)}{(k d)^{n+\frac{1}{2}}}+\sum_{1}^{\infty}(-1)^{m}(2 m-1) ! !\left(\frac{d}{b}\right)^{2 m} \frac{J_{m+n+\frac{1}{2}}(k d)}{(k d)^{m+n+\frac{1}{2}}}\right] }
\end{aligned}
$$

where $F F_{n}\left(\frac{d}{b}, k d\right)$ denotes "form factor" mentioned before. We can verify that

$$
\begin{gathered}
F F_{n}\left(\frac{d}{b}, k d\right) \simeq 1 \text { as } k d \rightarrow 0 \text { and } \frac{d}{b} \rightarrow 0, \\
F F_{n}\left(\frac{d}{b}, k a\right) \simeq(k d)^{-n-1} \text { as } k d \rightarrow \infty \text { and } \frac{d}{b}<1 .
\end{gathered}
$$

The series in the form factor is uniformly convergent in the whole range of $k d$ if $d / b<1$. The fast convergence of series can be seen in Fig. 3.2 which shows that even the first term alone is a very good approximation. The first term contribution arises from the phase variation along the hole with the source current being far from the hole. The amplitude of the source field is constant in the hole and is equal to the 
value at the center of the hole. We may approximate the form factor as

$$
F F_{n}(k d) \simeq \sqrt{\frac{\pi}{2}}(2 n+1) ! ! \frac{J_{n+\frac{1}{2}}(k d)}{(k d)^{n+\frac{1}{2}}} .
$$

We now express $\mathcal{Z}^{(1)}$ in a form which is convenient for numerical evaluation,

$$
\mathcal{Z}^{(1)}=j \frac{4 Z_{0} d^{2} H_{0}^{2}}{9}(k d) \frac{F F_{1}^{2}}{F_{11}(k d)+k d F_{11}^{\prime}(k d)} .
$$

Numerical values of $\mathcal{Z}^{(1)}$ can be obtained by substituting Eqs. (3.49) and (3.53) into Eq. (3.56). Since all the functions used in the expression are tabulated in standard reference materials, it is easy to do; the result is shown in Fig. 3.3.

From the figure, we observe that the resonance frequency $k_{r}$ occurs at $k d=1.6$ where $\operatorname{Im} Z(k)$ is equal to zero by definition. Since all the electromagnetic power is dissipated at the resonance, $\operatorname{Re} Z(k)$ must have a maximum value there. But the figure shows that the maximum of $\operatorname{Re} Z(k)$ occurs at $k d=1.3$ which contradicts physical argument. Such an unphysical phenomenon may be corrected if we include the tangential field due to the incident electric field in Bethe's solution in our trial fields.

In order to investigate the impedance in the low frequency range, we use the expansions [19]

$$
\begin{aligned}
I_{11}(\alpha) & =\frac{2 \alpha^{3}}{27 \pi}-\frac{4 \alpha^{5}}{675 \pi}+\frac{16 \alpha^{7}}{55125 \pi}-\cdots \\
R_{11}(\alpha) & =\frac{1}{3}-\frac{\alpha^{2}}{15}+\frac{\alpha^{4}}{140}-\cdots
\end{aligned}
$$

We may write the impedance functional in a form appropriate to small values of $k d$ :

$$
\begin{aligned}
\operatorname{Re} \mathcal{Z}^{(1)} & =\frac{32 Z_{0} d^{2} H_{0}^{2}}{27 \pi}(k d)^{4}\left(1+\frac{22}{25}(k d)^{2}-\cdots\right) \\
\operatorname{Im} \mathcal{Z}^{(1)} & =\frac{4 Z_{0} d^{2} H_{0}^{2}}{3}(k d)\left(1+\frac{2}{5}(k d)^{2}-\cdots\right) .
\end{aligned}
$$


If we compare these with the low frequency result, namely $Z(k)=j\left(2 Z_{0} d^{3} H_{0}^{2} / 3\right) k$, we find that the result from the variational method is a factor of two larger than the low frequency result. This may be explained again by the fact that we excluded the tangential field in the hole due to the electric field in our trial fields.

Since the impedance shown in Fig. 3.3 is similar to the impedance of a parallel RLC-resonator circuit, it would be useful if we described our impedance functional in terms of circuit parameters. The impedance of a broadband RLC-resonator circuit is

$$
Z_{b b}(\omega)=\frac{R}{1+j Q\left(\frac{\omega}{\omega_{r}}-\frac{\omega_{r}}{\omega}\right)},
$$

where $Q$ is the quality factor and $\omega_{r}$ is the resonant frequency. In the limit of low frequency,

$$
Z_{b b}(\omega) \simeq j R\left(\omega / \omega_{r}\right) / Q .
$$

The quality factor $Q$ is defined by

$$
Q=\frac{\omega_{r}}{2 \triangle \omega}
$$

where $\left|Z_{b b}(\omega)\right|$ at the frequency $\omega=\omega_{r}+\Delta \omega$ is 0.707 of its maximum value.

From the above definition, we find that

$$
\omega_{r} \cong \frac{1.6 c}{d}, Q \cong 1.8, R \cong 3.84 Z_{0} d^{2} H_{0}^{2} .
$$

The second-order variational solution of impedance $\mathcal{Z}^{(2)}$ can be written as

$$
\begin{aligned}
\mathcal{Z}^{(2)}= & j \frac{4 Z_{0} d^{2} H_{0}^{2}}{9}(k d) \\
& \frac{\left(F_{22}-k d F_{22}^{\prime}\right) F F_{1}^{2}-\frac{k^{2} d^{2}}{25}\left[\left(F_{11}+k d F_{11}^{\prime}\right) F F_{2}^{2}-10 F_{12}^{\prime} F F_{1} F F_{2}\right]}{\left(F_{11}+k d F_{11}^{\prime}\right)\left(F_{22}-k d F_{22}^{\prime}\right)+\left(k d F_{12}^{\prime}\right)^{2}} .
\end{aligned}
$$

Numerical values of $\mathcal{Z}^{(2)}$ are shown in Fig. 3.4. The same comments as in Fig. 3.3 apply here also. 
In conclusion, we have derived the formula for the coupling impedance of a hole in an infinite screen based on a variational method. Our results show that the hole is acting like a broadband resonator circuit and our formulas are suitable for determining the circuit parameters which are of practical use. However, in using one type of trial field, it is shown that the resulting impedance exhibits unphysical behavior. Such a deficiency of the method is not removed by simply going to the higher order approximation. We therefore believe that, in order to obtain physically consistent results, we should include another type of trial field, namely the field inside the hole due to the electric field which appeared in Bethe's low frequency solution. This is carried out in the next section. 

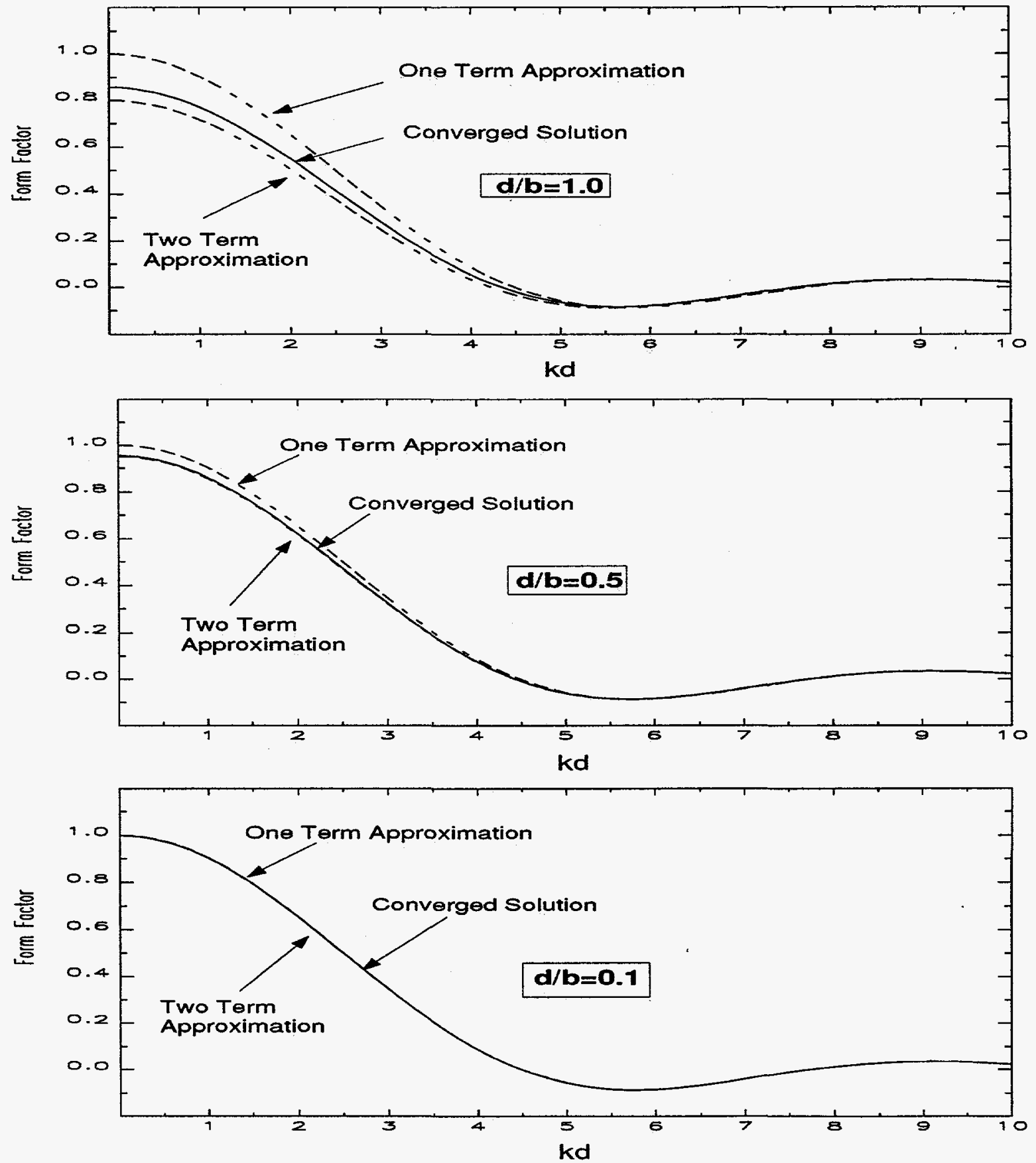

Figure 3.2: Form factor as functions of $k d$. 

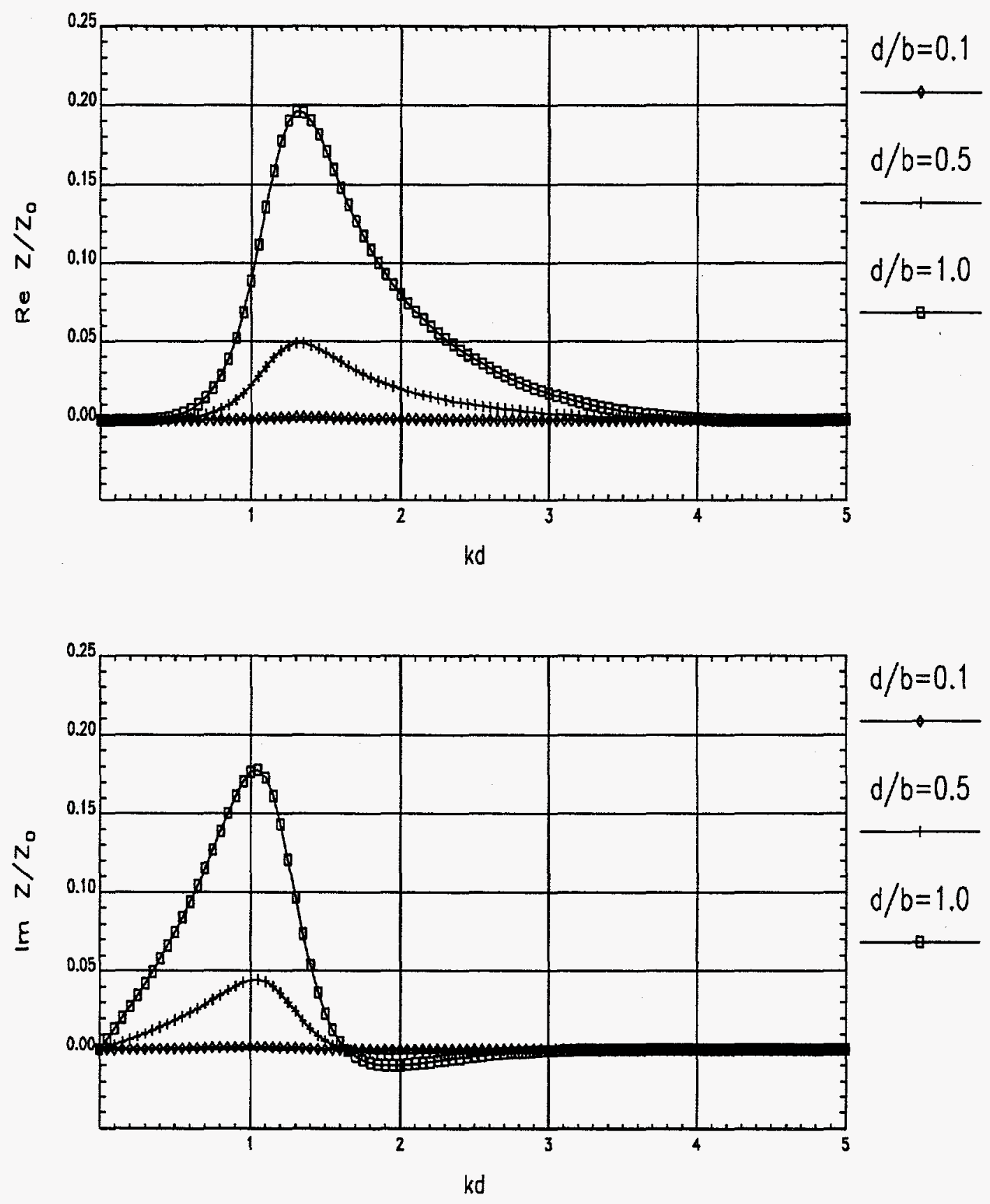

Figure 3.3: Impedance Functional $\mathcal{Z}^{(1)}(k d)$ for various $d / b$ values. 

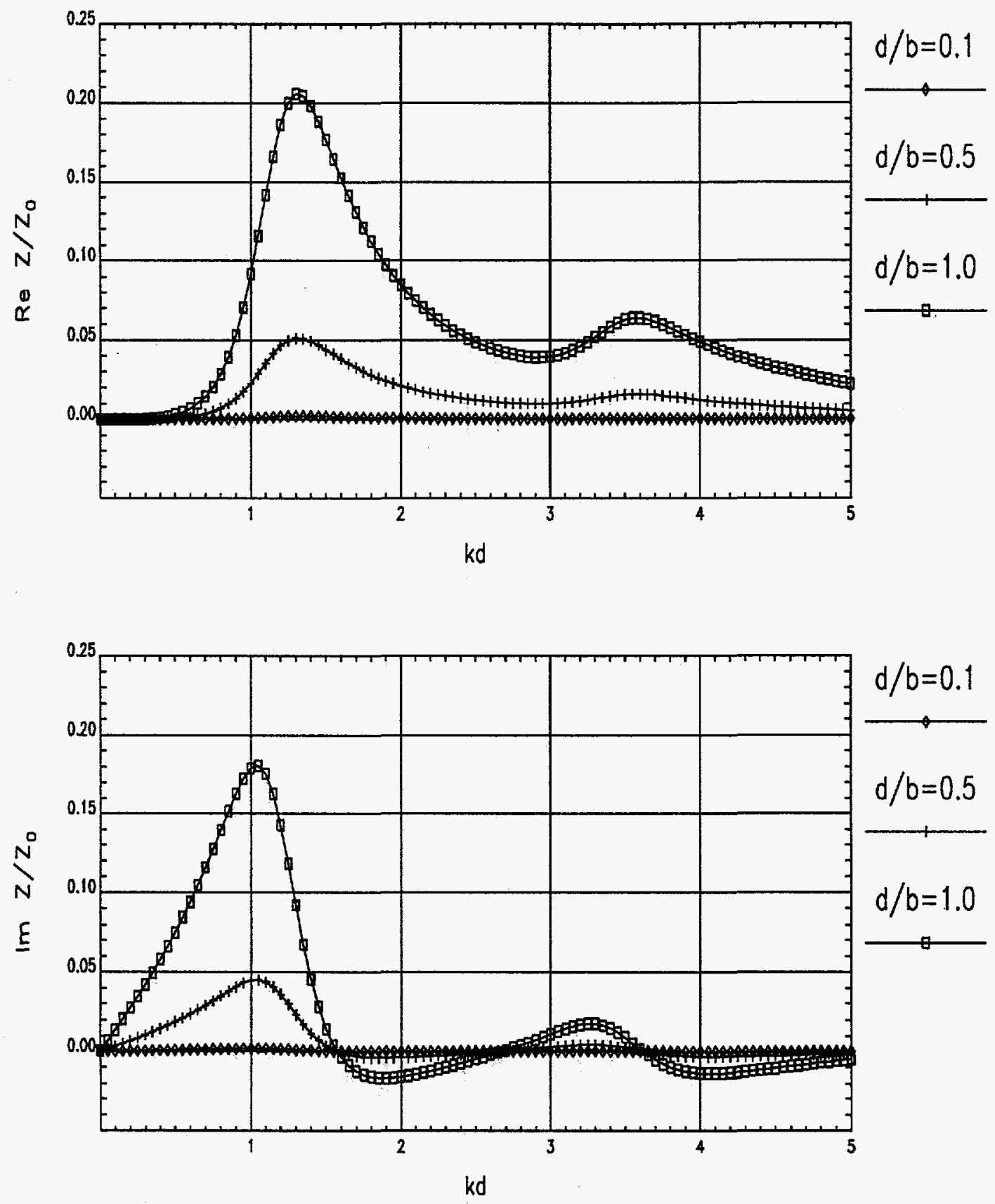

Figure 3.4: Impedance Functional $\mathcal{Z}^{(2)}(k d)$ for various $d / b$ values. 


\subsubsection{The Second Variational Solution}

In order to eliminate the unphysical behavior of the longitudinal coupling impedance found in the previous section, we include the tangential field in the hole due to the incident electric field in our variational calculation. An appropriate two-component trial field in the hole based on Bethe's solution is taken to be

$$
\mathbf{n} \times \mathbf{E}=a_{1} \sqrt{1-(\rho / d)^{2}} \mathbf{e}_{x}+b_{1} \frac{(\rho / d)}{\sqrt{1-(\rho / d)^{2}}} \mathbf{e}_{\theta}
$$

Since the impedance functional $\mathcal{Z}(k)$ is

$$
\mathcal{Z}(k)=\frac{1}{4 j \omega \epsilon} \frac{\left[\int_{S_{a}} \mathbf{H}^{i} \cdot(\mathbf{n} \times \mathbf{E}) d S\right]^{2}}{\int_{S_{a} \int_{S_{a}}}[\mathbf{n} \times \mathbf{E}(\mathbf{r})] \cdot \overline{\mathbf{G}}_{0}\left(\mathbf{r} \mid \mathbf{r}^{\prime}\right) \cdot\left[\mathbf{n} \times \mathbf{E}\left(\mathbf{r}^{\prime}\right)\right] d S d S^{\prime}},
$$

we may write the numerator as

$$
\left(a_{1} B_{1}^{a}+b_{1} B_{1}^{b}\right)^{2}
$$

and the denominator as

$$
4 j \omega \epsilon\left[a_{1}^{2} C_{11}^{a a}+a_{1} b_{1} C_{11}^{a b}+b_{1}^{2} C_{11}^{b b}\right]
$$

where

$$
\begin{aligned}
B_{1}^{a}= & \int_{S_{a}} H_{x}^{i}(\rho, \theta) \sqrt{1-(\rho / d)^{2}} d S, \\
B_{1}^{b}= & \int_{S_{a}} H_{x}^{i}(\rho, \theta) \frac{(\rho / d)}{\sqrt{1-(\rho / d)^{2}}} d S, \\
C_{11}^{a a}= & \int_{0}^{d} \sqrt{1-(\rho / d)^{2}} \rho d \rho \int_{0}^{d} \sqrt{1-\left(\rho^{\prime} / d\right)^{2}} \rho^{\prime} d \rho^{\prime} \int_{0}^{2 \pi} d \theta \int_{0}^{2 \pi} d \theta^{\prime} \\
& {\left[\sin \theta \sin \theta^{\prime} G_{\rho \rho^{\prime}}+\sin \theta \cos \theta^{\prime} G_{\rho \theta^{\prime}}+\cos \theta \sin \theta^{\prime} G_{\theta \rho^{\prime}}+\cos \theta \cos \theta^{\prime} G_{\theta \theta^{\prime}}\right], }
\end{aligned}
$$




$$
\begin{aligned}
C_{11}^{a b}= & \int_{0}^{d} \sqrt{1-(\rho / d)^{2}} \rho d \rho \int_{0}^{d} \frac{\left(\rho^{\prime} / d\right)}{\sqrt{1-\left(\rho^{\prime} / d\right)^{2}}} \rho^{\prime} d \rho^{\prime} \\
& \int_{0}^{2 \pi} d \theta \int_{0}^{2 \pi} d \theta^{\prime}\left[\sin \theta G_{\rho \theta^{\prime}}+\cos \theta G_{\theta \theta^{\prime}}\right] \\
+ & \int_{0}^{d} \sqrt{1-\left(\rho^{\prime} / d\right)^{2}} \rho^{\prime} d \rho^{\prime} \int_{0}^{d} \frac{(\rho / d)}{\sqrt{1-(\rho / d)^{2}}} \rho d \rho \\
& \int_{0}^{2 \pi} d \theta \int_{0}^{2 \pi} d \theta^{\prime}\left[\sin \theta^{\prime} G_{\theta \rho^{\prime}}+\cos \theta^{\prime} G_{\theta \theta^{\prime}}\right] \\
C_{11}^{b b}= & \int_{0}^{d} \frac{(\rho / d)}{\sqrt{1-(\rho / d)^{2}}} \rho d \rho \int_{0}^{d} \frac{\left(\rho^{\prime} / d\right)}{\sqrt{1-\left(\rho^{\prime} / d\right)^{2}}} \rho^{\prime} d \rho^{\prime} \int_{0}^{2 \pi} d \theta \int_{0}^{2 \pi} d \theta^{\prime} G_{\theta \theta^{\prime}} .
\end{aligned}
$$

The components of dyadic Green's function in the cylindrical coordinate system are defined by

$$
\begin{aligned}
& G_{\rho \rho^{\prime}}=\left(\cos \left(\theta-\theta^{\prime}\right)-\frac{1}{k^{2}} \frac{\partial^{2}}{\partial \rho \partial \rho^{\prime}}\right) G, \\
& G_{\rho \theta^{\prime}}=\left(\sin \left(\theta-\theta^{\prime}\right)-\frac{1}{k^{2} \rho^{\prime}} \frac{\partial^{2}}{\partial \rho \partial \theta^{\prime}}\right) G, \\
& G_{\theta \rho^{\prime}}=\left(-\sin \left(\theta-\theta^{\prime}\right)-\frac{1}{k^{2} \rho} \frac{\partial^{2}}{\partial \theta \partial \rho^{\prime}}\right) G, \\
& G_{\theta \theta^{\prime}}=\left(\cos \left(\theta-\theta^{\prime}\right)-\frac{1}{k^{2} \rho \rho^{\prime}} \frac{\partial^{2}}{\partial \theta \partial \theta^{\prime}}\right) G,
\end{aligned}
$$

where $G$ is the free-space scalar Green's function which can be expressed as

$$
\begin{aligned}
G & =\frac{e^{-j k R}}{4 \pi R}=\frac{1}{4 \pi} \int_{0}^{\infty} \frac{q d q}{\sqrt{q^{2}-k^{2}}} J_{0}(q R) \\
& =\frac{1}{4 \pi} \sum_{0}^{\infty}\left(2-\delta_{0 n}\right) \cos n\left(\theta-\theta^{\prime}\right) \int_{0}^{\infty} \frac{q d q}{\sqrt{q^{2}-k^{2}}} J_{n}(q \rho) J_{n}\left(q \rho^{\prime}\right)
\end{aligned}
$$


The impedance functional, in terms of these coefficients, may be written as

$$
\mathcal{Z}(k)=\frac{\left(a_{1} B_{1}^{a}+b_{1} B_{1}^{b}\right)^{2}}{4 j \omega \epsilon\left[a_{1}^{2} C_{11}^{a a}+a_{1} b_{1} C_{11}^{a b}+b_{1}^{2} C_{11}^{b b}\right]}
$$

or

$$
4 j \omega \epsilon\left[a_{1}^{2} C_{11}^{a a}+a_{1} b_{1} C_{11}^{a b}+b_{1}^{2} C_{11}^{b b}\right] \mathcal{Z}(k)=\left(a_{1} B_{1}^{a}+b_{1} B_{1}^{b}\right)^{2}
$$

A stationary property of $\mathcal{Z}$, i.e., $\partial \mathcal{Z} / \partial a_{1}=0$, yields

$$
2 a_{1} C_{11}^{a a}+b_{1} C_{11}^{a b}=-j \frac{Z_{0}}{2 k} B_{1}^{a}
$$

and, similarly from $\partial \mathcal{Z} / \partial b_{1}=0$, we have

$$
a_{1} C_{11}^{a b}+2 b_{1} C_{11}^{b b}=-j \frac{Z_{0}}{2 k} B_{1}^{b}
$$

Solving two linear algebraic equations, we obtain

$$
\begin{aligned}
a_{1} & =j \frac{Z_{0}}{2 k} \frac{C_{11}^{a b} B_{1}^{b}-2 C_{11}^{b b} B_{1}^{a}}{4 C_{11}^{a a} C_{11}^{b b}-\left(C_{11}^{a b}\right)^{2}}, \\
b_{1} & =j \frac{Z_{0}}{2 k} \frac{C_{11}^{a b} B_{1}^{a}-2 C_{11}^{a a} B_{1}^{b}}{4 C_{11}^{a a} C_{11}^{b b}-\left(C_{11}^{a b}\right)^{2}} .
\end{aligned}
$$

Once the unknown coefficients $a_{1}(k)$ and $b_{1}(k)$ are determined, the impedance functional can be calculated from the definition

$$
\mathcal{Z}(k)=a_{1} B_{1}^{a}+b_{1} B_{1}^{b}
$$

We can also calculate the longitudinal coupling impedance which may be written as

$$
Z(k)=a_{1} B_{1}^{a *}+b_{1} B_{1}^{b *}
$$


We first evaluate $C_{11}^{b b}$. The corresponding integral in angle variables becomes

$$
\begin{aligned}
\int_{0}^{2 \pi} \int_{0}^{2 \pi} d \theta d \theta^{\prime} G_{\theta \theta^{\prime}}= & \frac{1}{4 \pi}\left[\int_{0}^{2 \pi} \int_{0}^{2 \pi} d \theta d \theta^{\prime} \cos (\theta-\theta) \sum_{\mathbf{0}}^{\infty}\left(2-\delta_{0 n}\right) \cos n\left(\theta-\theta^{\prime}\right)\right. \\
& \left.-\int_{0}^{2 \pi} \int_{0}^{2 \pi} d \theta d \theta^{\prime} \frac{1}{k^{2} \rho \rho^{\prime}} \frac{\partial^{2}}{\partial \theta \partial \theta^{\prime}} \sum_{0}^{\infty}\left(2-\delta_{0 n}\right) \cos n\left(\theta-\theta^{\prime}\right)\right] \\
& \int_{0}^{\infty} \frac{q d q}{\sqrt{q^{2}-k^{2}}} J_{1}(q \rho) J_{1}\left(q \rho^{\prime}\right) .
\end{aligned}
$$

From the orthogonal property of trigonometric functions, we get

$$
\int_{0}^{2 \pi} \int_{0}^{2 \pi} d \theta d \theta^{\prime} G_{\theta \theta^{\prime}}=\pi \int_{0}^{\infty} \frac{q d q}{\sqrt{q^{2}-k^{2}}} J_{1}(q \rho) J_{1}\left(q \rho^{\prime}\right) .
$$

If we define

$$
I_{1}^{b}=\int_{0}^{d} \frac{(\rho / d)}{\sqrt{1-(\rho / d)^{2}}} J_{1}(q \rho) \rho d \rho
$$

we have

$$
C_{11}^{b b}=\pi \int_{0}^{\infty} \frac{q d q}{\sqrt{q^{2}-k^{2}}} I_{1}^{b} I_{1}^{b} .
$$

A similar procedure yields

$$
\begin{aligned}
C_{11}^{a b} & =0 \\
C_{11}^{a a} & =\frac{\pi}{2} \int_{0}^{\infty} \frac{q d q}{\sqrt{q^{2}-k^{2}}}\left[I_{0}^{a} I_{0}^{a}+I_{2}^{a} I_{2}^{a}-\frac{q^{2}}{k^{2}} I_{0}^{a} I_{0}^{a}\right],
\end{aligned}
$$

where $I_{0}^{a}$ and $I_{2}^{a}$ are defined by

$$
\begin{aligned}
& I_{0}^{a}=\int_{0}^{d} \sqrt{1-\left(\frac{\rho}{d}\right)^{2}} J_{0}(q \rho) \rho d \rho \\
& I_{2}^{a}=\int_{0}^{d} \sqrt{1-\left(\frac{\rho}{d}\right)^{2}} J_{2}(q \rho) \rho d \rho .
\end{aligned}
$$

Since $C_{11}^{a b}=0$, the unknown coefficients $a_{1}$ and $b_{1}$ are simplified to

$$
a_{1}=-j \frac{Z_{0}}{4 k} \frac{B_{1}^{a}}{C_{11}^{a a}}, \quad b_{1}=-j \frac{Z_{0}}{4 k} \frac{B_{1}^{b}}{C_{11}^{b b}} .
$$


We further note that

$$
4 j \omega \epsilon a_{1}^{2} C_{11}^{a a}=4 j \omega \epsilon \int_{S_{a}} \int_{S_{a}}\left(a_{1} \sqrt{1-(\rho / d)^{2}}\right) G_{x x}\left(\rho, \theta \mid \rho^{\prime}, \theta^{\prime}\right)\left(a_{1} \sqrt{1-\left(\rho^{\prime} / d\right)^{2}}\right) d S d S^{\prime}
$$

which indicates that

$$
C_{11}^{a a}=C_{11} / 2
$$

where $C_{11}$ is defined in Eq. (3.26), Section 3.3.2. We also note that $B_{1}^{a}$ is equal to $B_{1}$ defined in Eq. (3.25), Section 3.3.2. Thus, we only need to evaluate $C_{11}^{b b}$ and $B_{1}^{b}$.

Before we carry out the necessary integrals for $C_{11}^{b b}$, we note that if we define

$$
\begin{aligned}
& Z_{H}(k)=a_{1} B_{1}^{a *}=-j \frac{Z_{0}}{4 k} \frac{\left|B_{1}^{a}\right|^{2}}{C_{11}^{a a}}=-j \frac{Z_{0}}{2 k} \frac{\left|B_{1}\right|^{2}}{C_{11}} \\
& Z_{E}(k)=b_{1} B_{1}^{b *}=-j \frac{Z_{0}}{4 k} \frac{\left|B_{1}^{b}\right|^{2}}{C_{11}^{b b}}
\end{aligned}
$$

$Z(k)$ can be written as

$$
Z(k)=Z_{E}(k)+Z_{H}(k)
$$

where $Z_{H}(k)$ is already calculated in Section 3.3.2. From this, we find that the tangential fields in the hole due to electric field and magnetic field contribute to the impedance separately.

In order to show the order of approximation, we adopt the following notation

$$
Z^{(N+M)}=Z_{E}^{(N)}+Z_{H}^{(M)}
$$

where $M$ or $N$ denotes the order of approximation or the number of terms used for the trial field, and $Z_{H}^{(N)}=Z^{(N)}$ is calculated in Section 3.3.2.

Using Sonine's first finite integral formula, we evaluate $I_{1}^{b}$ as follows,

$$
I_{1}^{b}=\int_{0}^{d} \frac{(\rho / d)}{\sqrt{1-\left(\rho^{2} / d^{2}\right)}} J_{1}(q \rho) \rho d \rho
$$




$$
\begin{aligned}
& =d^{2} \int_{0}^{\pi / 2} J_{1}(q d \sin \theta) \sin ^{2} \theta d \theta \\
& =d^{2} \sqrt{\frac{\pi}{2}} \frac{J_{3 / 2}(q d)}{(q d)^{1 / 2}} .
\end{aligned}
$$

Substituting the above into Eq. (3.78), we obtain

$$
C_{11}^{b b}=\frac{\pi^{2} d^{3}}{2} \int_{0}^{\infty} \frac{J_{3 / 2}^{2}(q d)}{\sqrt{q^{2}-k^{2}}} d q
$$

Introducing a new variable $\alpha=k d$, we separate the above into two parts, that is,

$$
C_{11}^{b b}=\frac{\pi^{2} d^{3}}{2} I=\frac{\pi^{2} d^{3}}{2}\left(I_{1}-I_{2}\right),
$$

where

$$
I_{1}=\int_{0}^{\infty} \frac{J_{3 / 2}(q) J_{1 / 2}(q)}{q \sqrt{q^{2}-\alpha^{2}}} d q, I_{2}=\int_{0}^{\infty} \frac{J_{3 / 2}(q) J_{-1 / 2}(q)}{\sqrt{q^{2}-\alpha^{2}}} d q .
$$

From the product representation of the Bessel function

$$
J_{\mu}(z) J_{\nu}(z)=\frac{2}{\pi} \int_{0}^{\pi / 2} J_{\mu+\nu}(2 z \cos \theta) \cos (\mu-\nu) \theta d \theta, \quad \operatorname{Re}(\mu+\nu)>-1,
$$

we find

$$
\begin{aligned}
& I_{1}=\frac{2}{\pi} \int_{0}^{\pi / 2} \cos \theta d \theta \int_{0}^{\infty} \frac{J_{2}(2 q \cos \theta)}{q \sqrt{q^{2}-\alpha^{2}}} d q, \\
& I_{2}=\frac{2}{\pi} \int_{0}^{\pi / 2} \cos 2 \theta d \theta \int_{0}^{\infty} \frac{J_{1}(2 q \cos \theta)}{\sqrt{q^{2}-\alpha^{2}}} d q .
\end{aligned}
$$

We first consider the real part of $I_{1}$.

$$
\begin{aligned}
\operatorname{Re} I_{1} & =\frac{2}{\pi} \int_{0}^{\pi / 2} \cos \theta d \theta \int_{\alpha}^{\infty} \frac{J_{2}(2 q \cos \theta)}{q \sqrt{q^{2}-\alpha^{2}}} d q \\
& =\frac{2}{\pi} \int_{0}^{\pi / 2} \cos \theta d \theta \sqrt{\frac{\pi}{2}} \frac{J_{3 / 2}(2 \alpha \cos \theta)}{(2 \cos \theta)^{1 / 2} \alpha^{3 / 2}}
\end{aligned}
$$

where we used the integral formula ${ }^{2}$

$$
\begin{aligned}
\int_{x}^{\infty} \frac{J_{\nu}(a x)}{x^{\nu-1}}\left(x^{2}-z^{2}\right)^{\mu} d x= & \frac{2^{\mu} \Gamma(\mu+1)}{a^{\mu+1} z^{\nu-\mu-1}} J_{\nu-\mu-1}(a z), \\
& a \geq 0, \operatorname{Re}\left(\frac{\nu}{2}-\frac{1}{4}\right)>\operatorname{Re} \mu>-1 .
\end{aligned}
$$

${ }^{2}$ See reference [30], p. 417 . 
When $\nu$ is half of an odd integer, the function $J_{\nu}(z)$ has a finite representation in terms of algebraic and trigonometric functions of $z$, i.e.,

$$
J_{3 / 2}(z)=\sqrt{\frac{2}{\pi z}}\left(\frac{\sin z}{z}-\cos z\right) .
$$

Then

$$
\operatorname{Re} I_{1}=\frac{1}{\pi \alpha^{2}} \int_{0}^{\pi / 2} d \theta\left[\frac{\sin (2 \alpha \cos \theta)}{2 \alpha \cos \theta}-\cos (2 \alpha \cos \theta)\right]
$$

If we define

$$
F_{1}(\alpha)=\int_{0}^{\pi / 2} \frac{\sin (2 \alpha \cos \theta)}{2 \cos \theta} d \theta
$$

we find

$$
R e I_{1}=\frac{1}{\pi \alpha^{2}}\left[\frac{F_{1}(\alpha)}{\alpha}-\frac{\pi}{2} J_{0}(2 \alpha)\right]
$$

Since

$$
F_{1}^{\prime}(\alpha)=\int_{0}^{\pi / 2} \cos (2 \alpha \cos \theta) d \theta=\frac{\pi}{2} J_{0}(2 \alpha)
$$

it follows that

$$
F_{1}(\alpha)=\int_{0}^{\alpha} F_{1}^{\prime}(t) d t=\frac{\pi}{2} \int_{0}^{\alpha} J_{0}(2 t) d t=\frac{\pi}{4} \int_{0}^{2 \alpha} J_{0}(t) d t .
$$

Thus we finally obtain the finite representation of $\operatorname{Re} I_{1}$ as

$$
R e I_{1}=\frac{1}{4 \alpha^{2}}\left[\frac{1}{\alpha} \int_{0}^{2 \alpha} J_{0}(t) d t-2 J_{0}(2 \alpha)\right] .
$$

We now consider the imaginary part of $I_{1}$.

$$
\begin{aligned}
\operatorname{Im} I_{1} & =-\frac{2}{\pi} \int_{0}^{\pi / 2} \cos \theta d \theta \int_{0}^{\alpha} \frac{J_{2}(2 q \cos \theta)}{q \sqrt{\alpha^{2}-q^{2}}} d q \\
& =-\frac{2}{\pi} \int_{0}^{\pi / 2} \cos \theta d \theta \int_{0}^{\alpha} \frac{J_{2}(2 \alpha \cos \theta \sin \phi)}{\alpha \sin \phi} d \phi \\
& =-\frac{2}{\pi} \int_{0}^{\pi / 2} \cos \theta d \theta \sqrt{\frac{\pi}{2}} \frac{S_{3 / 2}(2 \alpha \cos \theta)}{(2 \cos \theta)^{1 / 2} \alpha^{3 / 2}}
\end{aligned}
$$


where we used the general Sonine's first finite integral formula ${ }^{3}$

$$
\int_{0}^{\pi / 2} J_{\mu}(z \sin \theta) \sin ^{1-\mu} \theta d \theta=\sqrt{\frac{\pi}{2 z}} S_{\mu-\frac{1}{2}}(z) .
$$

$S_{\nu}(z)$ is Struve's function which has a finite representation if $\nu$ is half of an odd integer, i.e.,

$$
S_{3 / 2}(z)=\sqrt{\frac{z}{2 \pi}}\left(1+\frac{2}{z^{2}}-\frac{2}{z} \sin z-\frac{2 \cos z}{z^{2}}\right) .
$$

With the substitution of a finite representation of Struve's functions, we obtain

$$
\begin{aligned}
\operatorname{Im} I_{1} & =-\frac{1}{\pi \alpha^{2}} \int_{0}^{\pi / 2} d \theta\left[\alpha \cos \theta-\sin (2 \alpha \cos \theta)+\frac{1-\cos (2 \alpha \cos \theta)}{2 \alpha \cos \theta}\right], \\
& =-\frac{1}{\pi \alpha^{2}}\left[\alpha-\frac{\pi}{2} S_{0}(2 \alpha)+\frac{F_{2}(\alpha)}{\alpha}\right]
\end{aligned}
$$

where we used

$$
\int_{0}^{\pi / 2} \sin (z \cos \theta) \sin ^{2 \nu} \theta d \theta=\frac{\sqrt{\pi} \Gamma\left(\nu+\frac{1}{2}\right)}{2\left(\frac{z}{2}\right)^{\nu}} S_{\nu}(z),
$$

and $F_{2}(\alpha)$ is defined as

$$
F_{2}(\alpha)=\int_{0}^{\pi / 2} \frac{1-\cos (2 \alpha \cos \theta)}{2 \cos \theta} d \theta
$$

It is evident that $F_{2}(0)=0$ and that

$$
F_{2}^{\prime}(\alpha)=\int_{0}^{\pi / 2} \sin (2 \alpha \cos \theta) d \theta=\frac{\pi}{2} S_{0}(2 \alpha) .
$$

From these it follows that

$$
F_{2}(\alpha)=\int_{0}^{\alpha} F_{2}^{\prime}(t) d t=\frac{\pi}{2} \int_{0}^{\alpha} S_{0}(2 t) d t=\frac{\pi}{4} \int_{0}^{2 \alpha} S_{0}(t) d t .
$$

Accordingly

$$
\text { Im } I_{1}=-\frac{1}{4 \alpha^{2}}\left[\frac{4}{\pi} \alpha-2 S_{0}(2 \alpha)+\frac{1}{\alpha} \int_{0}^{2 \alpha} S_{0}(t) d t\right] .
$$

\footnotetext{
${ }^{3}$ See reference $[30]$, p. 374 .
} 
Following the similar procedure, we obtain a finite representation of $I_{2}$ as

$$
\begin{aligned}
\operatorname{Re} I_{2} & =\frac{1}{2 \alpha}\left[2 J_{1}(2 \alpha)-\int_{0}^{2 \alpha} J_{0}(t) d t\right] \\
\operatorname{Im} I_{2} & =-\frac{1}{2 \alpha}\left[2 S_{1}(2 \alpha)-\int_{0}^{2 \alpha} S_{0}(t) d t\right] .
\end{aligned}
$$

Since $I=I_{1}-I_{2}$, we find that

$$
\begin{aligned}
& \operatorname{Re} I=+\left(\frac{1}{4 \alpha^{3}}+\frac{1}{2 \alpha}\right) \int_{0}^{2 \alpha} J_{0}(t) d t-\frac{J_{0}(2 \alpha)}{2 \alpha^{2}}-\frac{J_{1}(\alpha)}{\alpha} \\
& \operatorname{Im} I=-\left(\frac{1}{4 \alpha^{3}}+\frac{1}{2 \alpha}\right) \int_{0}^{2 \alpha} S_{0}(t) d t+\frac{S_{0}(2 \alpha)}{2 \alpha^{2}}+\frac{S_{1}(\alpha)}{\alpha}-\frac{1}{\pi \alpha}
\end{aligned}
$$

Substituting these equations into Eq. (3.91), we obtain the expression for $C_{11}^{b b}$. For the small values of $\alpha$, we may obtain a series expansion which can be written as

$$
I(\alpha)=\frac{1}{3}\left(1+\frac{\alpha^{2}}{5}-\frac{9 \alpha^{4}}{140}+\cdots\right)-j \frac{4 \alpha^{3}}{27 \pi}\left(1-\frac{4 \alpha^{2}}{25}+\cdots\right) .
$$

We now need to calculate $B_{1}^{b}$ to complete the expression for the impedance. Assuming $\mathbf{H}^{i}=H_{0} e^{-j k z} \mathbf{e}_{x}$ where $H_{0}$ is the field at the hole center, we obtain

$$
\begin{aligned}
B_{1}^{b} & =H_{0} \int_{0}^{d} \rho d \rho \int_{0}^{2 \pi} d \theta \frac{(\rho / d)}{\sqrt{1-(\rho / d)^{2}}} \cos \theta e^{-j k \rho \cos \theta} \\
& =-j 2 \pi H_{0} \int_{0}^{d} \frac{(\rho / d)}{\sqrt{1-(\rho / d)^{2}}} J_{1}(k \rho) \rho d \rho \\
& =-j 2 \pi d^{2} H_{0} \sqrt{\frac{\pi}{2}} \frac{J_{3 / 2}(k d)}{(k d)^{1 / 2}} .
\end{aligned}
$$

With the substitution of $C_{11}^{b b}$ and $B_{1}^{b}$ into the expression for $Z_{E}^{(1)}$, Eq. (3.87), we find

$$
Z_{E}^{(1)}=-j \frac{Z_{0} \pi H_{0}^{2}}{k^{2}} \frac{J_{3 / 2}^{2}(k d)}{I(k d)}
$$


It may be interesting to compare $Z_{E}^{(1)}(k)$ and $Z_{H}^{(1)}$ expanded in powers of $k d$. We find that

$$
\begin{aligned}
& Z_{H}^{(1)}=\frac{32 Z_{0} d^{2} H_{0}^{2}}{27 \pi}(k d)^{4}\left(1+\frac{22}{25}(k d)^{2}-\cdots\right)+j \frac{4 Z_{0} d^{2} H_{0}^{2}}{3}(k d)\left(1+\frac{2}{5}(k d)^{2}-\cdots\right), \\
& Z_{E}^{(1)}=\frac{8 Z_{0} d^{2} H_{0}^{2}}{27 \pi}(k d)^{4}\left(1-\frac{19}{25}(k d)^{2}+\cdots\right)-j \frac{2 Z_{0} d^{2} H_{0}^{2}}{3}(k d)\left(1-\frac{2}{5}(k d)^{2}+\cdots\right) .
\end{aligned}
$$

In the low frequency range, it is found

$$
Z^{(2)}=Z_{H}^{(1)}+Z_{E}^{(1)} \simeq j \frac{2 Z_{0} d^{3} H_{0}^{2}}{3} k,
$$

which is the same as the low frequency result found in Section 3.2.

Numerical results of $Z_{H}^{(1)}$ and $Z_{E}^{(1)}$ are presented in Fig. 3.5. There we find that the impedance of magnetic type, $Z_{H}$, is mainly inductive $(\operatorname{Im} Z>0)$, and the electric type exhibits capacitive behavior $(\operatorname{Im} Z<0)$.

Combining the two effects, we have numerical results of $Z^{(2)}(k)$ which are shown in Fig. 3.6. We note that the resonant frequency is located at $k d=1.35$. We also find that, at the resonant frequency, the imaginary part of the impedance is zero and the real part has its maximum which is consistent with physical argument. With this new result, we revise the circuit parameters, Eq. (3.62), found in Section 3.3.2 to the new one

$$
\omega_{r} \cong \frac{1.35 c}{d}, Q \cong 1.8, R \cong 1.62 Z_{0} d^{2} H_{0}^{2} .
$$

When we use the trial field which consists of the three terms

$$
\mathbf{n} \times \mathbf{E}=a_{1}\left(1-\frac{\rho^{2}}{d^{2}}\right)^{1 / 2} \mathbf{e}_{x}+a_{2}\left(1-\frac{\rho^{2}}{d^{2}}\right)^{3 / 2} \mathbf{e}_{x}+b_{1} \frac{(\rho / d)}{\sqrt{1-(\rho / d)^{2}}} \mathbf{e}_{\theta}
$$

we have

$$
Z^{(3)}=Z_{H}^{(2)}+Z_{E}^{(1)}
$$


Its numerical values are shown in Fig. 3.7.

In conclusion, we have shown that, with the inclusion of the tangential field due to the incident electric field in the trial fields, the result is consistent with the physical argument. 

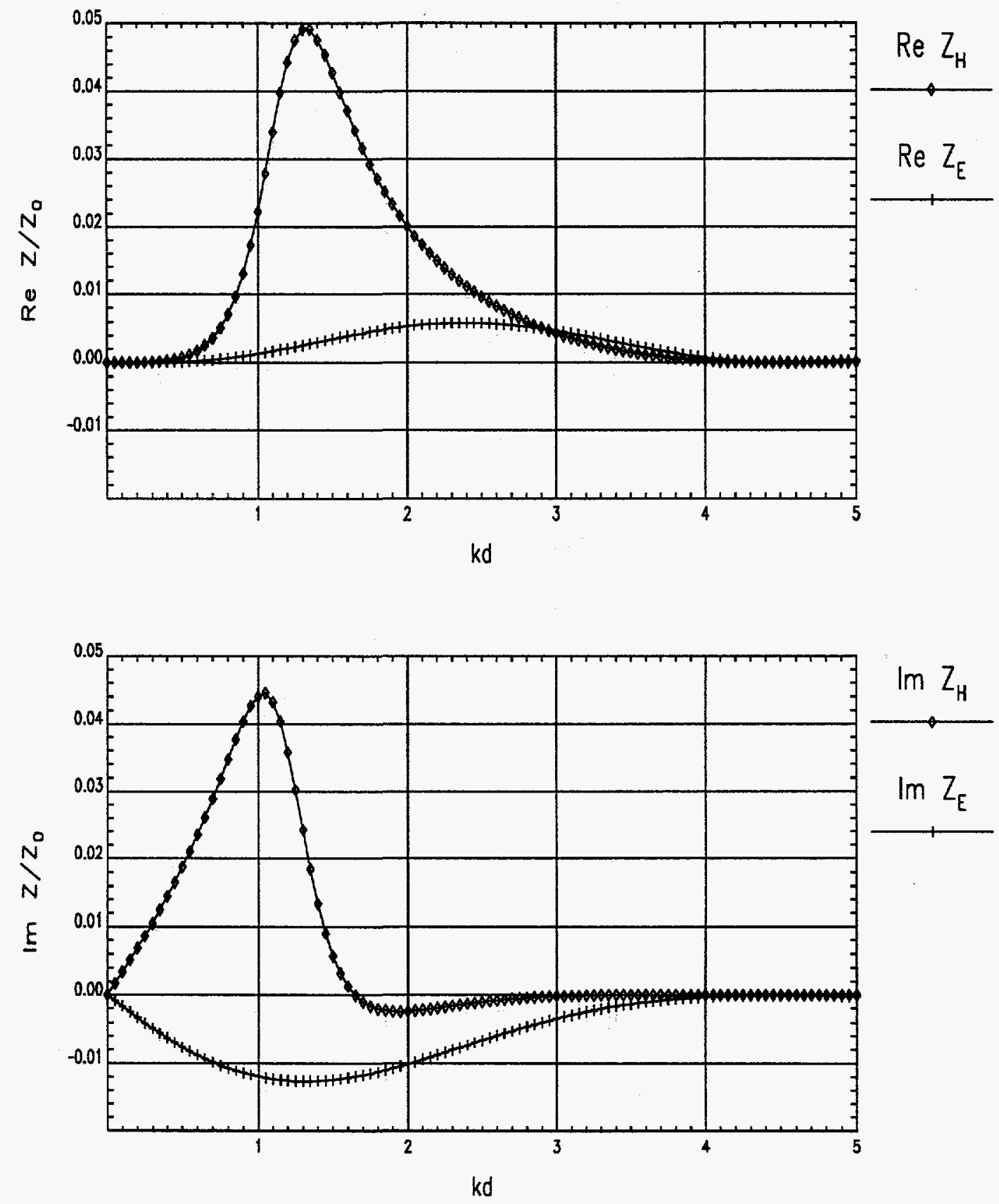

Figure 3.5: Longitudinal coupling impedance, $Z_{H}^{(1)}(k d)$ and $Z_{E}^{(1)}(k d)$, for $d / b=0.5$. 

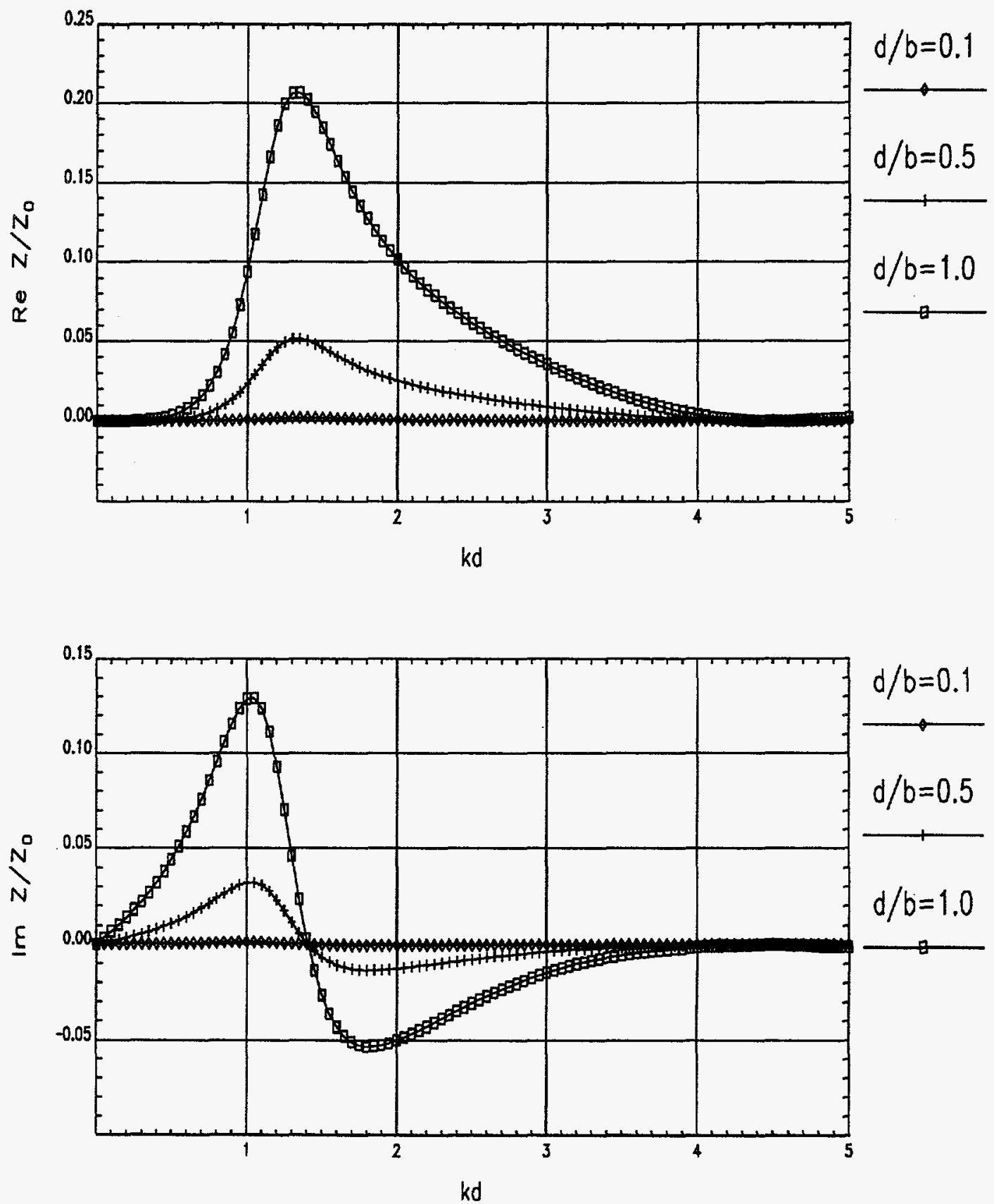

Figure 3.6: Longitudinal coupling impedance $Z^{(2)}(k d)$ for various $d / b$ values. 

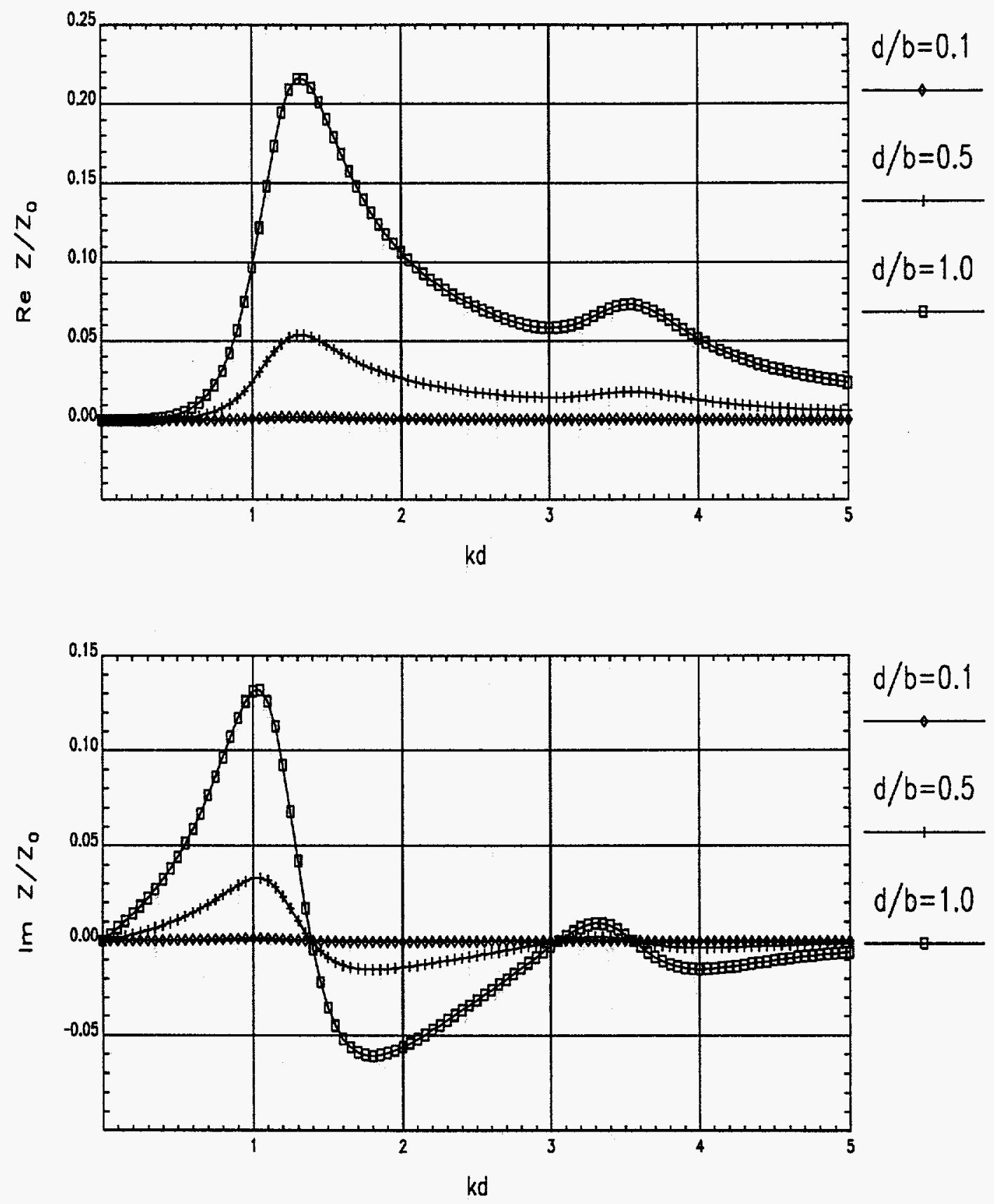

Figure 3.7: Longitudinal coupling impedance $Z^{(3)}(k d)$ for various $d / b$ values. 


\section{Chapter 4}

\section{Longitudinal Impedance of a Hole in the Accelerator Beam Pipe}

\subsection{Introduction}

In this chapter, we evaluate the longitudinal coupling impedance of an aperture in the accelerator beam pipe. Although the beam pipe can have an arbitrary crosssection, we assume a beam pipe with a rectangular cross-section which is a good approximation to the elliptical shape used in most of the electron accelerators and storage rings.

Since the aperture connects two regions, inside and outside of the beam pipe, we need to know the outside geometry to complete the specification of the problem. In a real accelerator, the region outside the beam pipe coupled by an aperture is completely arbitrary; it can be a closed vacuum vessel, waveguide structure, transmission line, or even a liner structure. Hence, in order to understand the fundamental characteristics of an aperture coupling, we choose the simplest geometry which has as many symmetries as possible.

These considerations result in the geometry of two equally shaped rectangular 


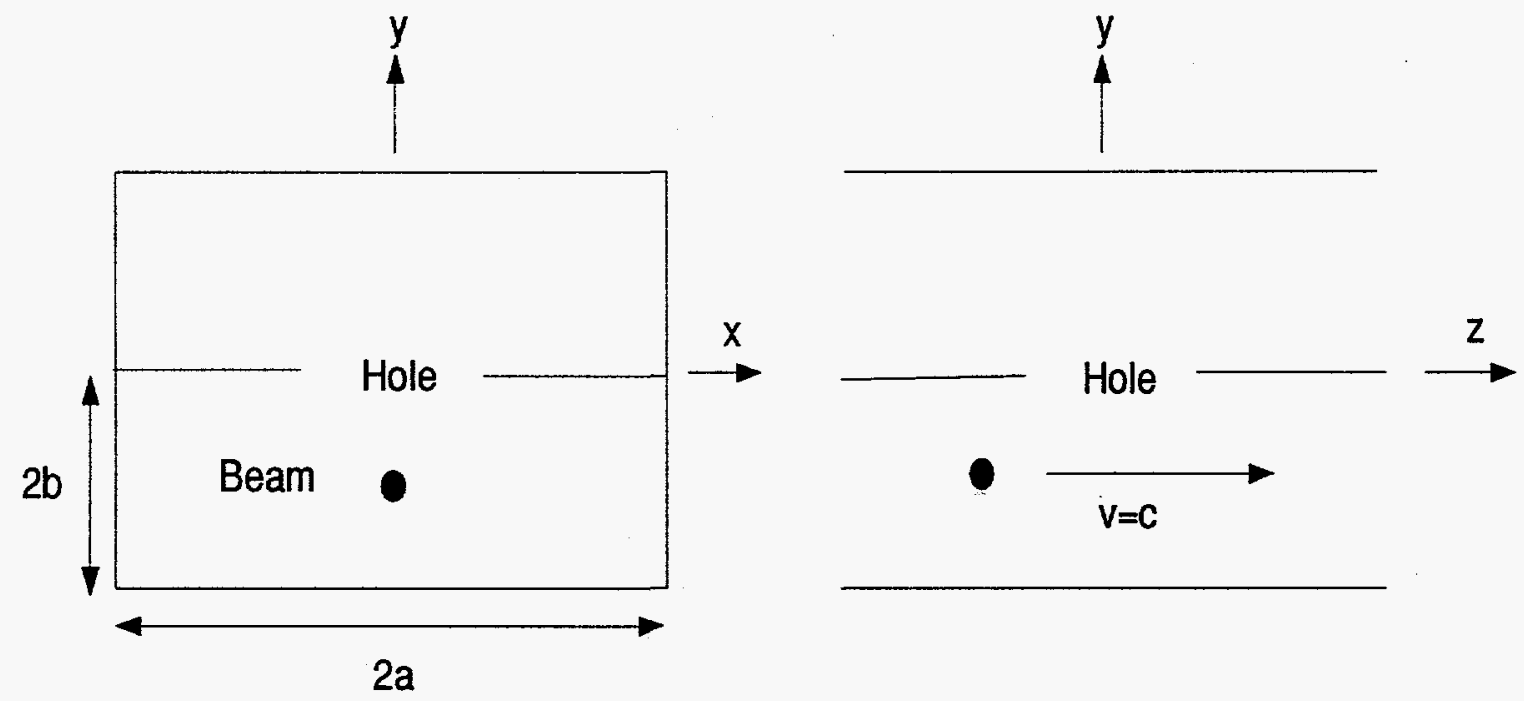

Figure 4.1: Rectangular wave guide coupled by a hole as a model to the accelerator beam pipe with a hole.

waveguides coupled by a hole which is located in the center of the common wall. Such a geometry is shown in Fig. 4.1.

In Section 4.2, we will estimate the impedance in the low frequency range using Bethe's small hole theory.

In Section 4.3, we will obtain the variational solutions of impedance using different trial fields and the results will be compared in Section 4.4 with the numerical calculation from the general wake potential program MAFIA-T3.

\subsection{Low Frequency Solution}

If the radius of a hole is much smaller than the wavelength, and the plane of the hole is the symmetry plane, we can use Bethe's result for the field inside hole (see 
Eq. (3.2)) which may be written as

$$
\mathbf{n} \times \mathbf{E}=-\frac{\rho}{\pi \sqrt{d^{2}-\rho^{2}}} \mathbf{e}_{\rho} \times \mathbf{E}_{0}+j \frac{2 k z_{0}}{\pi} \sqrt{d^{2}-\rho^{2}} \mathbf{H}_{0}
$$

where $d$ is the radius of the hole shown in Fig. 4.1, $k=\omega \sqrt{\epsilon_{0} \mu_{0}}$ is the free-space wave number, and $Z_{0}=377 \Omega$ is the characteristic impedance of free space.

Since we have already obtained a general expression for the impedance in the low frequency range as

$$
Z(k)=j \frac{2 Z_{0} d^{3} H_{0}^{2}}{3} k
$$

we only need to know the source field, $H_{0}$, at the hole center.

The electrostatic potential of line charge of density $\lambda$ located at $x=x_{1}, y=y_{1}$ satisfies the Poisson equation

$$
\frac{\partial^{2} \Phi}{\partial x^{2}}+\frac{\partial^{2} \Phi}{\partial y^{2}}=-\frac{\lambda}{\epsilon} \delta\left(x-x_{1}\right) \delta\left(y-y_{1}\right),
$$

where $\lambda / \epsilon$ can be written in terms of the drive current, $I_{0}$, as $\lambda / \epsilon=Z_{0} I_{0}$.

For the rectangular waveguide with walls at $x= \pm a, y= \pm b$, the corresponding Green's function satisfies

$$
\frac{\partial^{2} G}{\partial x^{2}}+\frac{\partial^{2} G}{\partial y^{2}}=-\delta\left(x-x_{1}\right) \delta\left(y-y_{1}\right)
$$

with the boundary condition $G=0$ at $x= \pm a, y= \pm b$. Expanding Green's function in single series, we assume

$$
G(x, y)=\sum_{1}^{\infty} a_{n}(y) b_{n}(x)
$$

where $a_{n}(y)$ is the amplitude function and $b_{n}(x)$ is the basis function which is similar to the Fourier series expansion of an arbitrary function. In a Cartesian coordinate 
system, we may choose $b_{n}(x)$ as

$$
b_{n}(x)=\frac{1}{\sqrt{a}} \sin \frac{n \pi}{2 a}(x+a)
$$

which vanishes at $x= \pm a$ and satisfies the orthonormal conditions,

$$
\int_{-a}^{a} b_{n}(x) b_{m}(x) d x=\delta_{m n}
$$

Substituting Eq. (4.5) into Eq. (4.4), we obtain

$$
\sum_{1}^{\infty}\left[\frac{d^{2} a_{n}(y)}{d y^{2}}-\left(\frac{n \pi}{2 a}\right)^{2} a_{n}(y)\right] b_{n}(x)=-\delta\left(x-x_{1}\right) \delta\left(y-y_{1}\right) .
$$

Multiplying both sides of the equation and integrating, we obtain

$$
\frac{d^{2} a_{m}(y)}{d y^{2}}-\left(\frac{m \pi}{2 a}\right)^{2} a_{m}(y)=-b_{m}\left(x_{1}\right) \delta\left(y-y_{1}\right) .
$$

Solving the above equation for $a_{m}(y)$ by the well-known procedure, we find the potential function as

$$
\begin{aligned}
\Phi(x, y)= & \frac{2 I_{0} Z_{0}}{\pi} \sum_{n=1}^{\infty} \frac{\sin \frac{n \pi}{2 a}(x+a) \sin \frac{n \pi}{2 a}\left(x_{1}+a\right)}{n \sinh \frac{n \pi b}{a}} \\
& \begin{cases}\sinh \frac{n \pi}{2 a}(y+b) \sinh \frac{n \pi}{2 a}\left(b-y_{1}\right) & y<y_{1} \\
\sinh \frac{n \pi}{2 a}(b-y) \sinh \frac{n \pi}{2 a}\left(y_{1}+b\right) & y>y_{1} .\end{cases}
\end{aligned}
$$

We note that, if we assumed $G=\sum a_{n}(x) b_{n}(y)$, we would have an alternative expression with $a$ and $b, x$ and $y, x_{1}$ and $y_{1}$ interchanged in the above equation.

Using the relation $H_{x}=-E_{y} / Z_{0}$, we obtain $H_{0}$ at the center of the hole $(x=$ $0, y=b, x_{1}=y_{1}=0$ )

$$
H_{0}=-\frac{I_{0}}{2 a} \sum_{n=o d d} \frac{1}{\cosh \frac{n \pi b}{2 a}} .
$$

This series converges fast. With this, we have

$$
Z(k)=j \frac{Z_{0} d^{3}}{6 a^{2}}\left(\sum_{n=o d d} \frac{1}{\cosh \frac{n \pi b}{2 a}}\right)^{2} k .
$$




\subsection{Variational Solution}

The general expression for impedance in variational method was derived in the previous chapter and was shown to be

$$
\mathcal{Z}(k)=-\frac{1}{2} \frac{\left[\int_{S_{a}} \mathbf{H}^{i} \cdot\left(\mathbf{n} \times \mathbf{E}^{a}\right) d S\right]^{2}}{\int_{S_{a}} \mathbf{H}^{a} \cdot\left(\mathbf{n} \times \mathbf{E}^{a}\right) d S},
$$

where $\mathbf{H}^{a}$ may be calculated as

$$
\mathbf{H}^{a}(\mathbf{r})=-j \omega \epsilon \int_{S_{a}} \overline{\mathbf{G}}_{m}\left(\mathbf{r} \mid \mathbf{r}^{\prime}\right) \cdot\left(\mathbf{n} \times \mathbf{E}^{a}\right) d S^{\prime}
$$

The dyadic Green's function $\overline{\mathbf{G}}_{m}$ is of magnetic type whose boundary condition should satisfy $\mathbf{n} \times \nabla \times \overline{\mathbf{G}}_{m}=\mathbf{0}$ on the walls. The eigenfunction expansion of dyadic Green's function for a rectangular waveguide is developed by Tai [29]. He shows that $\overline{\mathbf{G}}_{m}$ can be expanded as a double-infinite series of harmonic functions in Cartesian coordinates $(x, y, z)$. Since the convergence of a series of harmonic functions is very slow and the Cartesian coordinate system suitable for the rectangular waveguide is not convenient in describing a hole in the wall, we use the image principle to remove the guide walls, enabling us to use the familiar free-space dyadic Green's function.

Such a conversion of the geometry from a hole in a waveguide to infinite image holes embedded in free-space is shown in Fig. 4.2. In the original problem, the center of an equivalent magnetic surface current (replacing the hole closed by the conducting wall) is located at the origin inside the waveguide. In the converted problem, waveguide walls are replaced by an infinite array of image magnetic surface currents ("image-source") located at $x= \pm 2 n a, n=1,2,3, \cdots$ and $y= \pm 4 m b, m=$ $1,2,3, \cdots$ in addition to the original magnetic surface current ("self-source"). 

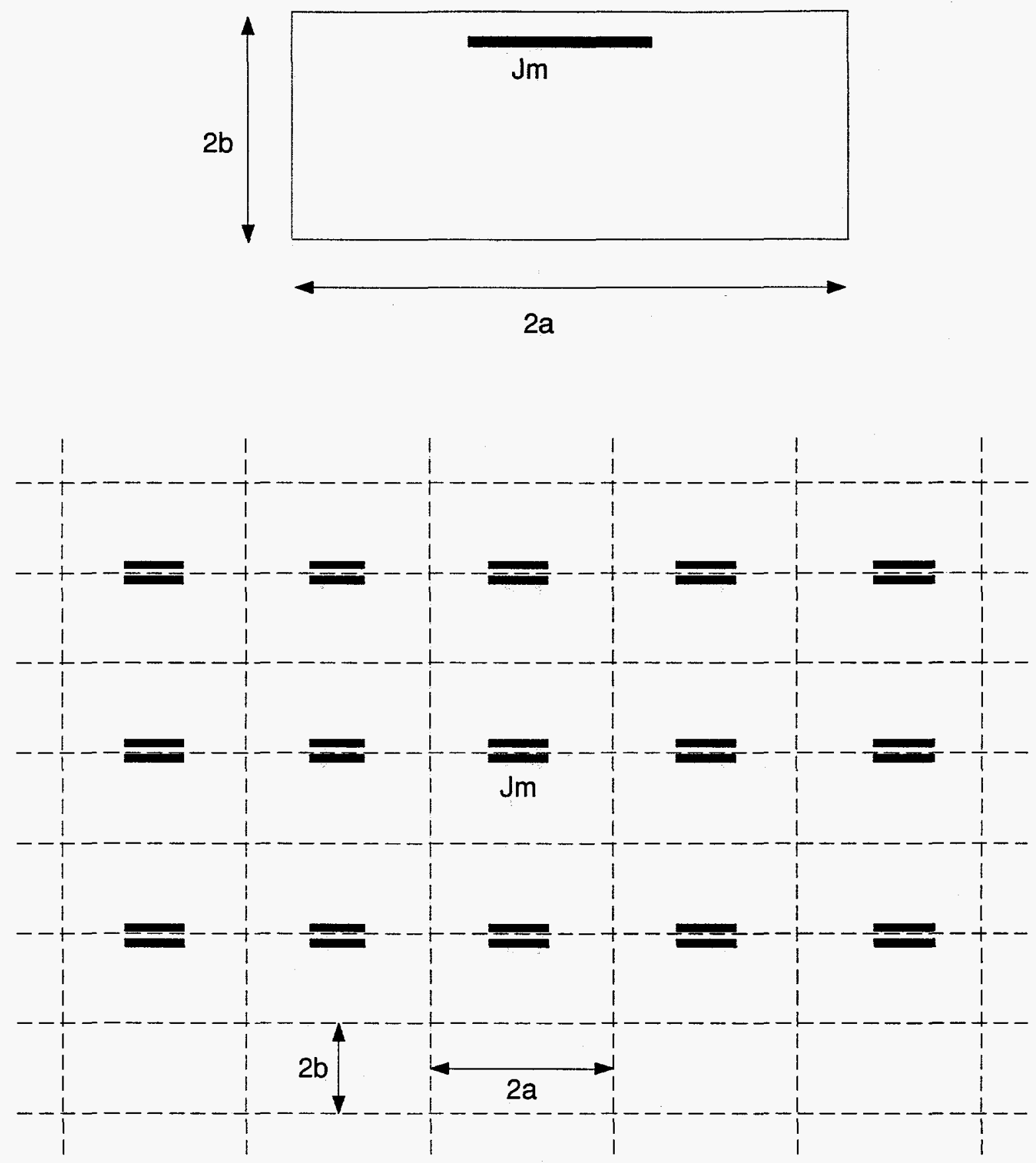

Figure 4.2: Hole in waveguide, its equivalent magnetic surface current and its images in infinite space. 
Since we have two contributions to $\mathbf{H}^{a}$ from the self- and image-sources, we separate $\mathbf{H}^{a}$ into two parts, namely,

$$
\mathbf{H}^{a}=\mathbf{H}_{\text {self }}^{a}+\mathbf{H}_{\text {image }}^{a} .
$$

We note that, if the contribution from images is zero, the impedance functional $\mathcal{Z}(k)$ is the same as the one for a hole in an infinite screen. The only difference is that the source field of the driving beam must be evaluated for waveguide structure. Thus, we calculate the contribution from the image-source and compare it with the contribution from the self-source.

For this purpose, we use a simple trial field used in Section 3.3.2 which is rewritten as

$$
\mathbf{J}_{m}=\mathbf{n} \times \mathbf{E}=\phi(\rho) \mathbf{e}_{x}, \phi(\rho)=\sqrt{1-\frac{\rho^{2}}{d^{2}}} .
$$

If we define the denominator in Eq. (4.13) as

$$
D=\int_{S_{a}}\left(\mathbf{H}_{\text {self }}^{a}+\mathbf{H}_{\text {image }}^{a}\right) \cdot\left(\mathbf{n} \times \mathbf{E}^{a}\right) d S=D_{\text {self }}+D_{\text {image }}
$$

then

$$
D_{s e l f}=-j \omega \epsilon \int_{S_{a}} \int_{S_{a}} \phi(\rho)\left(G_{x x}+G_{z z}\right) \phi\left(\rho^{\prime}\right) d S d S^{\prime}=-j \omega \epsilon C_{11},
$$

where $C_{m n}$ is calculated in Eq. (3.40). For small $k d, D_{\text {self }}$ has a series expansion in the form

$$
\begin{aligned}
\text { Im } D_{\text {self }} & =\frac{\pi^{2} d^{2}}{6 Z_{0}}\left(\frac{1}{\alpha}-\frac{3}{5} \alpha+\cdots\right), \\
\text { Re } D_{\text {self }} & =-\frac{4 \pi d^{2}}{27 Z_{0}} \alpha^{2}\left(1-\frac{3}{25} \alpha^{2}+\cdots\right),
\end{aligned}
$$

with $\alpha=k d$. In the low frequency range, the imaginary part of $D_{\text {self }}$ is dominant which results in $Z(k) \sim j k$. Thus, when we compare $D_{\text {self }}$ with the contribution from the image sources, $D_{\text {image }}$, we compare the imaginary part at the low frequency. 
For $D_{\text {image }}$, we use the small hole approximation so that the image-source is replaced by the $x$-directed magnetic dipole moment $M$. The dipole moment may be calculated using a general relation

$$
\int_{S_{a}} \mathbf{J}_{m} d S=-\int_{S_{a}} \mathbf{r} \nabla \cdot \mathbf{J}_{m} d S=j \omega \int_{S_{a}} \mathbf{r} \rho_{m} d S=j \omega \mu \mathbf{M},
$$

which results in $j \omega \mu \mathbf{M}=\frac{4 \pi d^{2}}{3} \mathbf{e}_{x}$ located at $x=2 n a, n=0, \pm 1, \pm 2, \cdots$ and $y=$ $2 m b, m=0, \pm 1, \pm 2, \cdots$, excluding the origin.

In order to calculate the magnetic field due to all image dipoles [9], it may be convenient to use the Hertzian potential of magnetic type, $\mathbf{\Pi}$. Since the magnetic dipole moment is $x$-directed, $\Pi$ has only an $x$ component which satisfies the wave equation

$$
\nabla^{2} \Pi_{x}+k^{2} \Pi_{x}=-M \text { and } H_{x}=k^{2} \Pi_{x}+\frac{\partial^{2} \Pi_{x}}{\partial x^{2}} .
$$

For a row of dipoles located at $y= \pm 4 m b, m=0,1,2, \cdots$, the potential becomes

$$
\Pi_{1}=\frac{M}{4 \pi} \sum_{m=-\infty}^{m=\infty} \frac{\exp \left[-j k \sqrt{x^{2}+(y-4 m b)^{2}+z^{2}}\right]}{\sqrt{x^{2}+(y-4 m b)^{2}+z^{2}}} .
$$

For this infinite sum, we use Poisson's sum formula defined as

$$
\sum_{n=-\infty}^{\infty} f(n \alpha)=\frac{1}{\alpha} \sum_{n=-\infty}^{\infty} F\left(\frac{2 n \pi}{\alpha}\right), \quad F(\omega)=\int_{-\infty}^{\infty} f(t) e^{j \omega t} d t .
$$

The required Fourier transformation is the integral of the type

$$
\begin{aligned}
I & =\int_{-\infty}^{\infty} e^{j q u} \frac{e^{-j k \sqrt{x^{2}+(y-u)^{2}}}}{\sqrt{x^{2}+(y-u)^{2}}} d u \\
& =e^{j q y} \int_{-\infty}^{\infty} e^{j q z} \frac{e^{-j k \sqrt{x^{2}+z^{2}}}}{\sqrt{x^{2}+z^{2}}} d z \\
& =e^{j q y} \int_{-\infty}^{\infty} e^{-x(q \cosh \theta+k \sinh \theta)} d \theta \\
& =e^{j q y} \int_{-\infty}^{\infty} e^{-x \sqrt{q^{2}-k^{2}} \cosh (\theta+\psi)} d \theta \\
& =2 e^{j q y} K_{0}\left[\sqrt{q^{2}-k^{2}} x\right]
\end{aligned}
$$


where $K_{0}(x)$ is the modified Bessel function of the second kind.

Thus, $\Pi_{1}$ becomes

$$
\Pi_{1}=\frac{M}{8 \pi b}\left\{K_{0}\left[j k \sqrt{x^{2}+z^{2}}\right]+2 \sum_{m=1}^{m=\infty} \cos \frac{m \pi y}{2 b} K_{0}\left[\Gamma_{0 m} \sqrt{x^{2}+z^{2}}\right]\right\}
$$

where $\Gamma_{0 m}=\left[(m \pi / 2 b)^{2}-k^{2}\right]$.

The potential arising from all the image dipoles is obtained by replacing $x$ by $x-2 n a$ in Eq. (4.26). Excluding the contribution from the dipole at the origin,

$$
\begin{aligned}
\Pi_{2} & =\frac{M}{8 \pi b} \sum_{n=-\infty}^{\infty} K_{0}\left[j k \sqrt{(x-2 n a)^{2}+z^{2}}\right] \\
& +\frac{M}{4 \pi b} \sum_{n=1}^{\infty} \sum_{m=1}^{\infty} \cos \frac{m \pi y}{2 b} K_{0}\left[\Gamma_{0 m} \sqrt{(x-2 n a)^{2}+z^{2}}\right] \\
& +\frac{M}{2 \pi} \sum_{m=1}^{\infty} \frac{\exp \left[-j k \sqrt{x^{2}+(y-4 m b)^{2}+z^{2}}\right]}{\sqrt{x^{2}+(y-4 m b)^{2}+z^{2}}}
\end{aligned}
$$

The first term is the contribution from the dipoles on the $x$-axis excluding the origin, the second term is from the dipoles not on the axis, and the third term is from the dipoles on the $y$-axis excluding the origin.

Since the evaluation of $H_{x}$ involves derivatives with respect to $x$ only, we may place $y$ and $z$ equal to zero which results in

$$
\begin{aligned}
\Pi_{3} & =\frac{M}{8 \pi b} \sum_{n=-\infty}^{\infty}{ }^{\prime} K_{0}[j k|x-2 n a|]+\frac{M}{4 \pi b} \sum_{n=1}^{\infty} \sum_{m=1}^{\infty} K_{0}\left[\Gamma_{0 m}|x-2 n a|\right] \\
& +\frac{M}{2 \pi} \sum_{m=1}^{\infty} \frac{\exp \left[-j k \sqrt{x^{2}+(4 m b)^{2}}\right]}{\sqrt{x^{2}+(4 m b)^{2}}}
\end{aligned}
$$

Assuming a low frequency in the range where $\Gamma_{0 m}$ is real, we may neglect the second term as $K_{0}(x)$ decreases exponentially when $x$ is real. But the first series converges very slowly so that we transform the series into the form suitable for using 
Poisson's sum formula,

$$
\frac{M}{8 \pi b} \sum_{n=\infty}^{\infty} K_{0}[j k|x-2 n a|]-\frac{M}{8 \pi b} K_{0}[j k|x|] .
$$

A necessary integral is 1

$$
\int_{-\infty}^{\infty} e^{j q u} K_{0}\left[j k \sqrt{(x-u)^{2}+z^{2}}\right] d u=\frac{\pi}{\sqrt{q^{2}-k^{2}}} e^{j q x-z \sqrt{q^{2}-k^{2}}}
$$

from which we obtain

$$
\frac{M}{8 \pi b} \sum_{n=-\infty}^{\infty} K_{0}[j k|x-2 n a|]=\frac{M}{16 a b} \frac{e^{j \frac{n \pi}{a} x}}{\Gamma_{0 n}}
$$

where $\Gamma_{0 n}=\sqrt{(n \pi / a)^{2}-k^{2}}$.

The final expression for $\Pi_{x}$ becomes

$$
\Pi_{x}=\frac{M}{16 a b} \sum_{n=-\infty}^{\infty} \frac{e^{j \frac{n \pi}{a} x}}{\Gamma_{0 n}}-\frac{M}{8 \pi b} K_{0}[j k|x|]+\frac{M}{2 \pi} \sum_{m=1}^{\infty} \frac{\exp \left[-j k \sqrt{x^{2}+(4 m b)^{2}}\right]}{\sqrt{x^{2}+(4 m b)^{2}}}
$$

For the field, $H_{x}$, we need $\partial^{2} \Pi_{x} / \partial x^{2}$ which is given by

$$
\begin{aligned}
\frac{\partial^{2} \Pi_{x}}{\partial x^{2}} & =-\frac{M}{16 a b} \sum_{n=-\infty}^{\infty}\left(\frac{n \pi}{a}\right)^{2} \frac{e^{j \frac{n \pi}{a} x}}{\Gamma_{0 n}}+\frac{M k^{2}}{8 \pi b}\left[K_{0}(j k x)+\frac{K_{1}(j k x)}{j k x}\right] \\
& +\frac{M}{2 \pi} \sum_{m=1}^{\infty} \frac{e^{-j k r_{m}}}{r_{m}}\left[\frac{j k x}{r_{m}^{2}}+\frac{2 x}{r_{m}^{3}}-\frac{k^{2} x}{r_{m}}+\frac{j k x}{r_{m}}-\left(j k+\frac{1}{r_{m}}\right)\left(\frac{1}{r_{m}}-\frac{x^{2}}{r_{m}^{3}}\right)\right]
\end{aligned}
$$

where $r_{m}=\sqrt{x^{2}+(4 m b)^{2}}$.

Thus, $H_{x}$ at the origin becomes

$$
\begin{aligned}
H_{x} & =k^{2} \Pi_{x}+\frac{\partial^{2} \Pi_{x}}{\partial x^{2}} \\
& =-\frac{M}{16 a b} \sum_{n=-\infty}^{\infty} \Gamma_{0 n} e^{j \frac{n \pi}{a} x}+\frac{M k^{2}}{8 \pi b} \frac{K_{1}(j k x)}{j k x} \\
& +\frac{M}{2 \pi}\left[k^{2} \sum_{1}^{\infty} \frac{e^{-j k 4 m b}}{4 m b}-\sum_{1}^{\infty} \frac{e^{-j k 4 m b}}{4 m b}\left(\frac{j k}{4 m b}+\frac{1}{(4 m b)^{2}}\right)\right]
\end{aligned}
$$

\footnotetext{
${ }^{1}$ See reference [13], p. 736 .
} 
Necessary formulas in evaluating the various geometric series in Eq. (4.33) are given in Table 4.1.

If $\Gamma_{0 n}$ is approximately taken to be $n \pi / a-k^{2} a / 2 \pi n$, the first series has the dominant part

$$
-\frac{M}{8 a b} \sum_{n=1}^{\infty} e^{j \frac{n \pi}{a} x}\left(\frac{n \pi}{a}-\frac{a k^{2}}{2 \pi n}\right)-j \frac{M}{16 a b} k
$$

which is readily summed to give

$$
\frac{M}{8 \pi b}\left[j\left(\frac{\pi k^{2}}{4}-\frac{\pi k}{2 a}\right)-\frac{k^{2}}{2} \ln 2 \sin \frac{\pi x}{2 a}+\frac{1}{x^{2}}\right],
$$

plus a correction part

$$
-\frac{M}{8 a b} \sum_{n=1}^{\infty}\left(\Gamma_{0 n}-\frac{n \pi}{a}+\frac{a k^{2}}{2 \pi n}\right)
$$

Thus the first series becomes

$$
\frac{M}{8 \pi b}\left[j\left(\frac{k^{2}}{4}-\frac{\pi k}{2 a}\right)-\frac{k^{2}}{2} \ln 2 \sin \frac{\pi x}{2 a}+\frac{1}{x^{2}}-\frac{\pi}{a} \sum_{n=1}^{\infty}(\cdots)\right] .
$$

For small $x$, the second term in $H_{x}$ becomes

$$
\frac{M}{8 \pi b}\left[\frac{k^{2}}{4}(2 \gamma-1)+\frac{k^{2}}{2} \ln \left(\frac{j k x}{2}\right)-\frac{1}{x^{2}}\right]
$$

where $\gamma=0.577 \cdots$ is Euler's constant. We notice that the singularity in $x$ vanishes as $x$ goes to zero.

The third series can be summed to give

$$
\frac{M}{8 \pi b}\left[\frac{k^{2}}{2}(\ln 4 k b-2 \ln 2 \sin 2 k b)-\frac{k^{2}}{4}-\frac{b^{2} k^{4}}{6}+j\left(\frac{4 b k^{3}}{3}-\frac{\pi k^{2}}{4}\right)\right] .
$$

Finally, $H_{x}$ at the origin is given as

$$
\begin{aligned}
\text { Re } H_{x} & =\frac{M}{8 \pi b}\left[(\ln 4 k b-2 \ln 2 \sin 2 k b+\gamma-1) \frac{k^{2}}{2}-\frac{b^{2}}{6} k^{4}-\frac{\pi}{a} \sum_{n=1}^{\infty}(\cdots)\right], \\
\operatorname{Im} H_{x} & =-\frac{M}{8 \pi b}\left(\frac{\pi}{2 a} k-\frac{4 b}{3} k^{3}\right),
\end{aligned}
$$




\begin{tabular}{llr}
\hline \hline$\sum_{1}^{\infty} e^{j n x}$ & $\frac{e^{j x}}{1-e^{j x}}=-\frac{1}{2}+\frac{j}{2} \cot \frac{x}{2}$, & $0<x<\pi$ \\
$\sum_{1}^{\infty} n e^{j n x}$ & $-\frac{1}{4} \csc ^{2} \frac{x}{2}=-\frac{1}{x^{2}}\left(1+\frac{x^{2}}{3}+\frac{87 x^{4}}{2880}+\cdots\right)$, & $0<x<\pi$ \\
$\sum_{1}^{\infty} \frac{e^{j n x}}{n}$ & $-\ln \left(1-e^{j x}\right)=-\ln 2 \sin \frac{x}{2}+j \frac{\pi-x}{2}$, & $0<x<2 \pi$ \\
$\sum_{1}^{\infty} \frac{e^{j n x}}{n^{2}}$ & $\frac{\pi^{2}}{6}-\frac{x}{4}(2 \pi-x)-j\left(x \ln x-x-\frac{x^{3}}{72} \cdots\right)$, & $0<x<2 \pi$ \\
$\sum_{1}^{\infty} \frac{e^{j n x}}{n^{3}}$ & $j\left(\frac{\pi^{2} x}{6}-\frac{\pi x^{2}}{4}+\frac{x^{3}}{12}\right)+\left(\frac{x^{2}}{2} \ln x-\frac{3 x^{2}}{4}-\frac{x^{4}}{288}-\cdots\right)+\sum_{n=1}^{\infty} \frac{1}{n^{3}}$, & $0<x<2 \pi$ \\
$\sum_{1}^{\infty} \frac{1}{n^{2}}$ & $\frac{\pi^{2}}{6}$ & \\
$\sum_{1}^{\infty} \frac{1}{n^{3}}$ & 1.202056903 & \\
\hline
\end{tabular}

Table 4.1: Geometric Series

which is valid for low frequencies.

With this field, we obtain

$$
\operatorname{Im} D_{\text {image }} \simeq-\frac{\pi^{2} d^{2}}{9 Z_{0}}\left(\frac{d}{b}\right) \alpha, \quad \alpha=k d .
$$

Comparing this with $D_{\text {self }}$, we see that the contribution to the impedance from image sources is negligible in the low frequency range if the hole size is small compared with the waveguide dimension, which is often the case in a typical accelerator beam pipe.

Thus, for the hole in a rectangular waveguide, we use the same analytic formulas obtained for the impedance of a hole in an infinite screen except that the source field appearing in the formula, Eq. (3.56), must be evaluated in the waveguide which is shown in Eq. (4.11). 


\subsection{Comparison with Numerical Results}

In order to verify our analytical results, we compare them with the numerical results from MAFIA-T3 which is a 3-D wake potential program. As mentioned in Chapter 1, MAFIA-T3 simply calculates the wake potential of the charge distributed in space, i.e., bunch potential. It assumes a bunch of charged particles in Gaussian distribution moving with velocity equal to the speed of light.

One of the geometries used in the MAFIA-T3 simulation is shown in Fig. 4.3 where we use a waveguide of width $2 a=2 \mathrm{~cm}$, height $2 b=1 \mathrm{~cm}$, the radius of a hole $d=4 \mathrm{~mm}$, and thickness of a hole plane $t=1 \mathrm{~mm}$. Although our method has been developed for a hole in a zero thickness plane, the numerical program cannot handle zero thickness.

The corresponding bunch potential together with the Gaussian bunch shape are shown in Fig. 4.4. We observe that the wake potential has a long tail similar to a damped harmonic oscillator. Such a long range wake potential results in an impedance with narrow bandwidth at a well-defined resonant frequency. Resonance phenomena are expected because the induced field in the hole bounded by the circular conducting edge will support a standing wave. When the hole size is reduced to $1 \mathrm{~mm}$, wake potential is quickly damped out after the bunch traverses the hole. Such a shortrange wake is shown in Fig. 4.5. Because of the short range in time, it will exhibit a broadband impedance in the frequency domain.

With this wake potential, we have to perform a Fourier transform in order to obtain the coupling impedance. The first step is to calculate $V(\omega)$ which is the direct Fourier transform of bunch potential from MAFIA-T3. For $d=4 m m$, the result is 
shown in Fig. 4.6.

After this step, we can obtain the coupling impedance by

$$
Z(\omega)=\frac{V(\omega)}{I(\omega)}, \quad I(\omega)=\exp \left[-\frac{1}{2}\left(\omega \sigma_{z} / c\right)^{2}\right],
$$

where $I(\omega)$ is the Fourier transform of the Gaussian bunch with rms bunch length $\sigma_{z}$.

In practice, $Z(\omega)$ can be computed only up to some limiting frequency because of the exponential factor in the equation. We choose the limiting frequency to be the one for which the exponential factor is five. Results for a hole of radius $4 \mathrm{~mm}$ are presented in Fig. 4.7 showing the resonance behavior with sharp peaks.

As a general rule, three-dimensional mesh programs such as MAFIA-T3 require a large amount of CPU time and a considerable memory size. In order to reduce the number of meshes, we take advantage of the symmetry in geometry. For example, instead of using full geometry as shown in Fig. 4.3 and running the program once with the boundary condition,

$$
\text { tangential electric field } \mathbf{E}_{\|}=0 \text { on the guide walls, }
$$

we may use only the bottom half of the geometry with two separate boundary conditions, $\mathbf{E}_{\|}=0$ and $\mathbf{H}_{\|}=0$ on the plane of the hole (symmetry plane). By doing this, we can save both the number of meshes and the CPU time.

Three different results, one using full geometry and the other two using half geometry with $\mathbf{E}_{\|}=0$ or $\mathbf{H}_{\|}=0$ are shown in Fig. 4.8. We notice that the impedance from the $\mathbf{E}_{\|}=0$ boundary condition is completely negligible. In fact, if the thickness becomes infinitesimal, its impedance should be zero. Thus, by utilizing the symmetry configuration, we only use half geometry with boundary condition $\mathbf{H}_{\|}=0$ and divide the result by a factor of two in order to achieve the same result as the one from full 
geometry. All results from MAFIA-T3 calculations shown in the later figures were obtained by this method.

We compare the coupling impedance from the variational method and from MAFIAT3 simulations for the holes with radius $1 \mathrm{~mm}, 2 \mathrm{~mm}, 4 \mathrm{~mm}$. The result for the hole with radius $1 \mathrm{~mm}$ is shown in Fig. 4.9. Since the ratio $d / b=0.2$ is small, our variational result, based on the assumption that the interaction between the hole and waveguide is small, is expected to be in good agreement with the numerical result which includes the effect of waveguide structure. From the MAFIA-T3 results, we find that the resonance occurs at the frequency $k d=1.8$. The variational result shows the resonance at $k d=1.35$. Although the predictions of resonant frequency from the two methods do not agree, results indicate that the resonance behavior is a property of the hole because the frequency of the dominant propagating mode is $16 \mathrm{GHz}$ for the $T M_{11}$ mode, which is far below the resonant frequency. We also observe that the variational result at the low frequency range is approximately a factor two larger than the numerical one. This may be explained as the thickness effect. Gluckstern [11] has shown that the coupling impedance of a hole in an infinite screen with finite thickness is reduced by a certain factor, which depends on the ratio $t / d$, compared to one in a zero-thickness screen. His result shows that the reduction factor for $t / d=1$ is 0.567 . If we include this factor in the variational result, we have a better agreement with the numerical one, as shown in Fig. 4.10. In this comparison we should note that, if the hole size is small compared with the waveguide dimension, the characteristics of coupling impedance can be explained by the consideration of a hole only.

For the hole with radius $2 \mathrm{~mm}$, the numerical result presented in Fig. 4.11 exhibits noisy peaks which, we believe, indicate the effects of the waveguide. However, its well- 
defined envelope shows the behavior of a broadband resonator with the resonance at $k d=1.8$, the same as the impedance for radius $1 \mathrm{~mm}$. Except for the location of the resonant frequency, the result from the variational method shows good agreement in magnitude. The reduction factor of 0.61 due to the thickness effect for $t / d=0.5$ is included in the variational result.

An interesting behavior is observed from the numerical results for the hole with radius $4 \mathrm{~mm}$. Since the ratio of a hole radius and waveguide half width is comparable, the waveguide effect may not be negligible any more. The result in Fig. 4.12 shows a strong interaction between hole and waveguide at the frequency of $16 \mathrm{GHz}$. It corresponds to a dominant propagating mode of $T M_{11} . T E_{m n}$ modes are not important because they do not contribute to the longitudinal coupling impedance. The reduction factor 0.70 for $t / d=0.25$ is taken into account in the variational result.

We have shown that the results from two different methods are in good agreement when the hole is small compared with the waveguide dimension. It indicates that, for a small hole, the effect from waveguide structure is minor because our variational method does not take into account such an effect. We also find that the thickness effect is not negligible unless $t / d<<1$. 


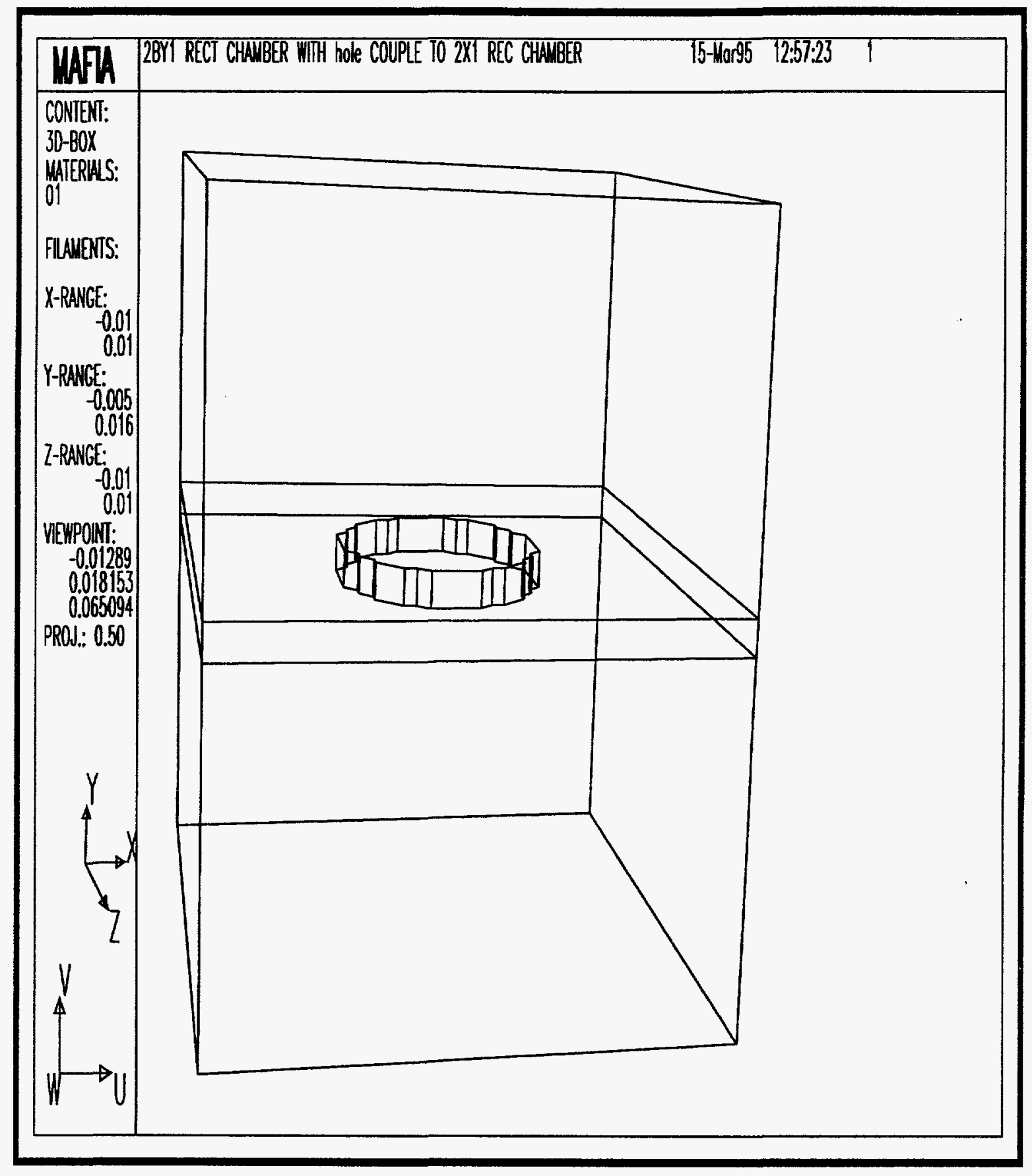

Figure 4.3: Geometry used in MAFIA-T3 simulation. 


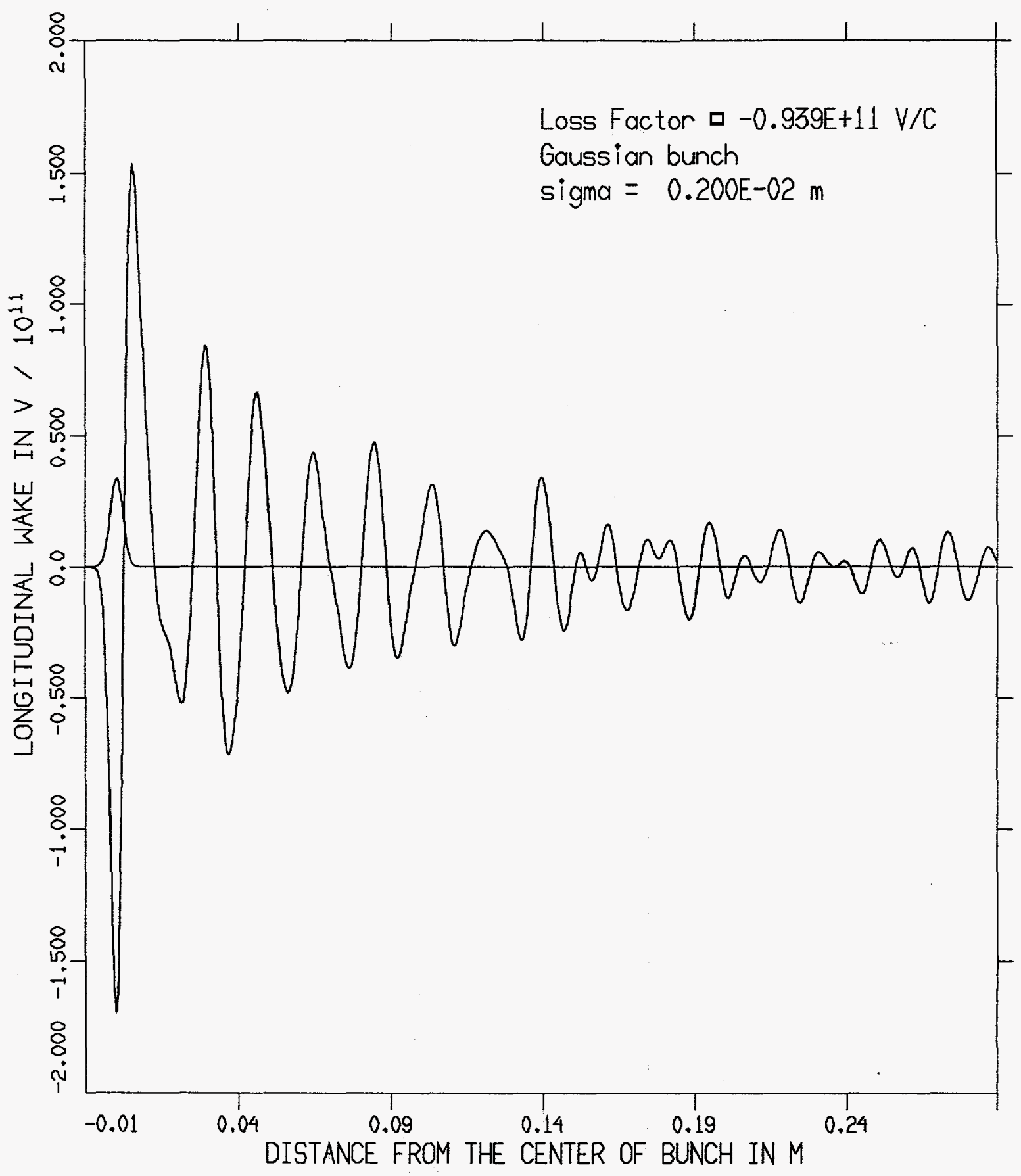

Figure 4.4: Bunch potential from MAFIA-T3 simulation for the hole with radius 4 $\mathrm{mm}$. 


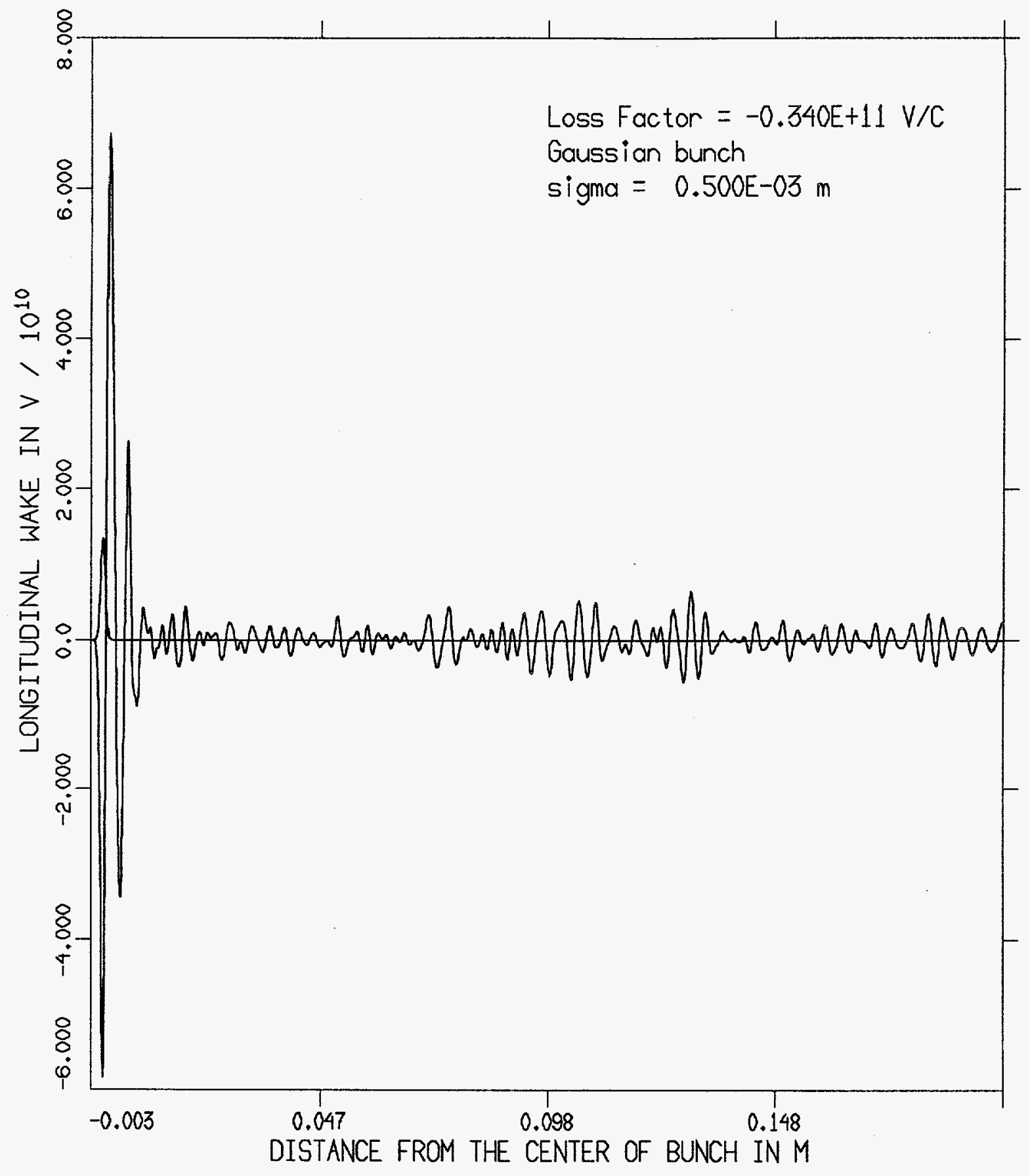

Figure 4.5: Bunch potential from MAFIA-T3 simulation for the hole with radius 1 $\mathrm{mm}$. 

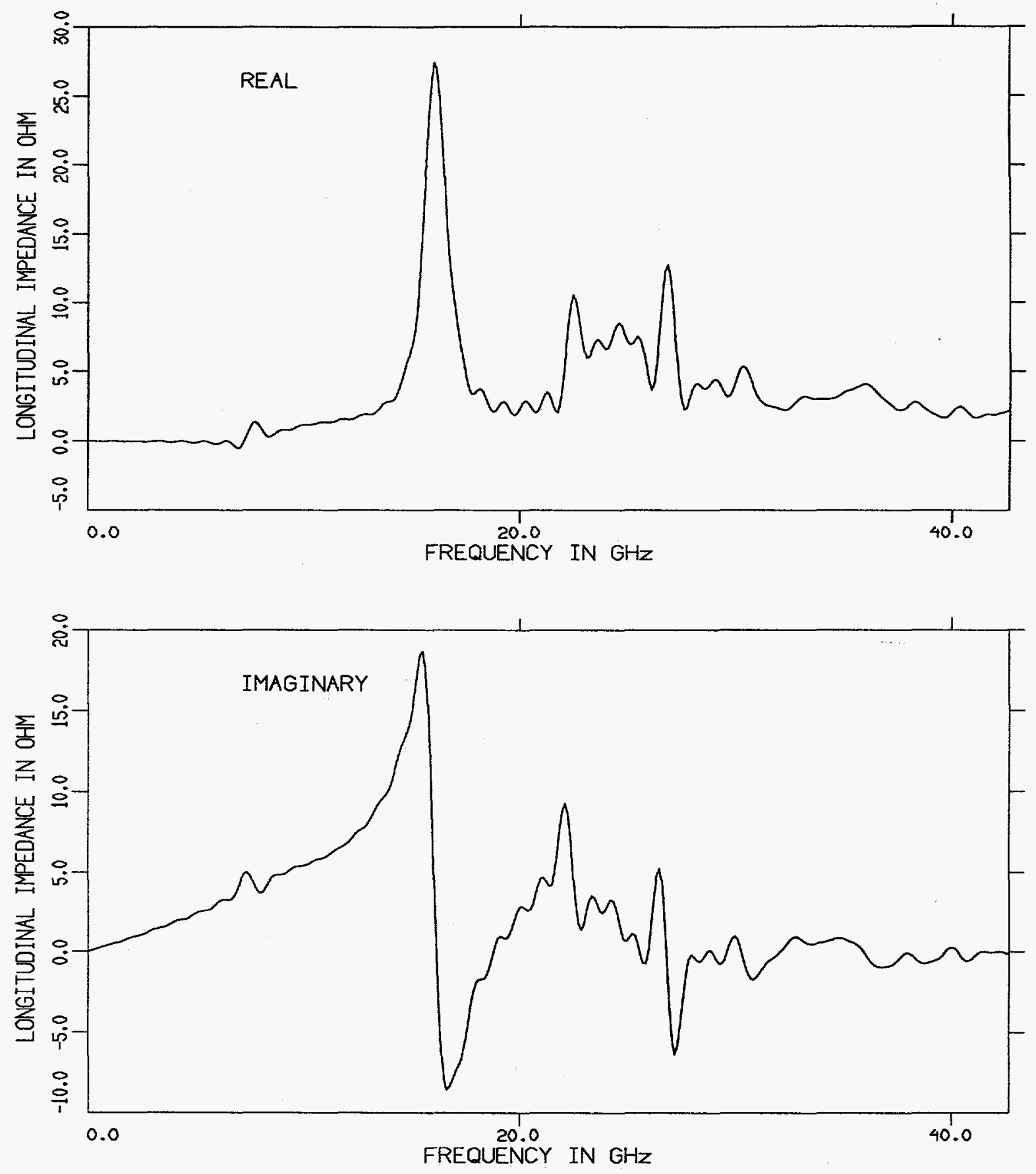

Figure 4.6: Fourier transform of bunch potential from MAFIA-T3 simulation for the hole with radius $4 \mathrm{~mm}$. 

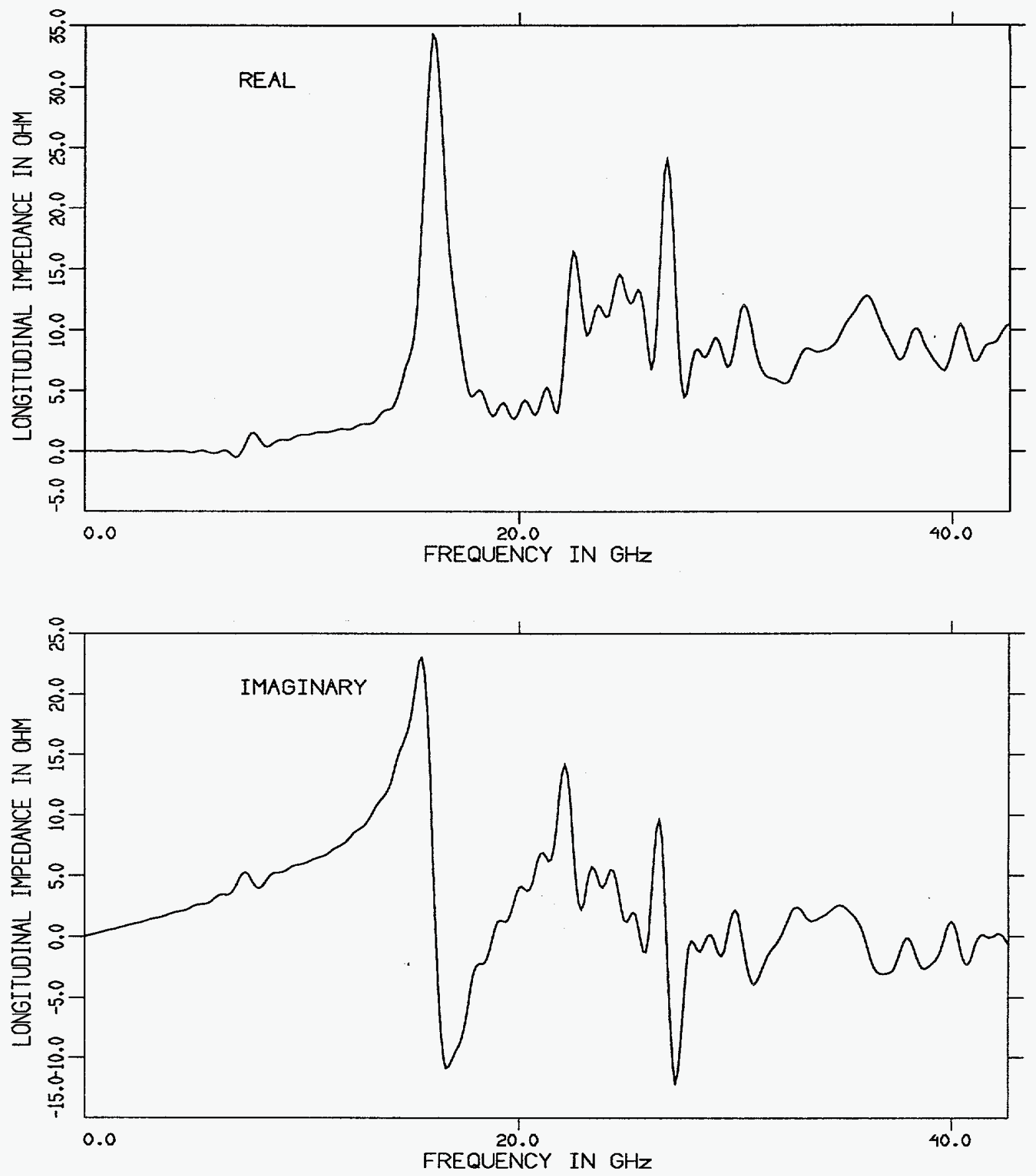

Figure 4.7: Coupling impedance of a point charge for the hole with radius $4 \mathrm{~mm}$. 

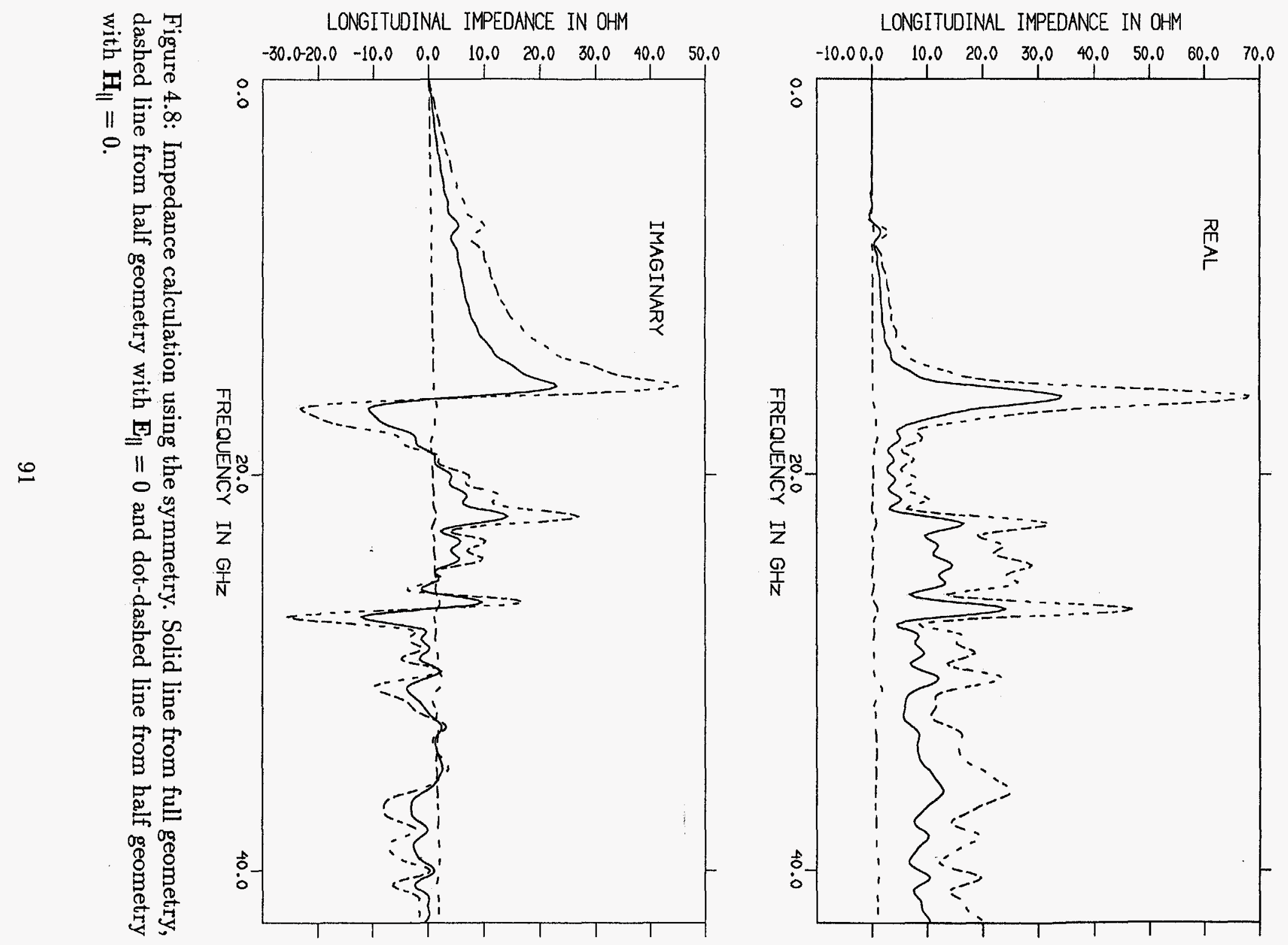

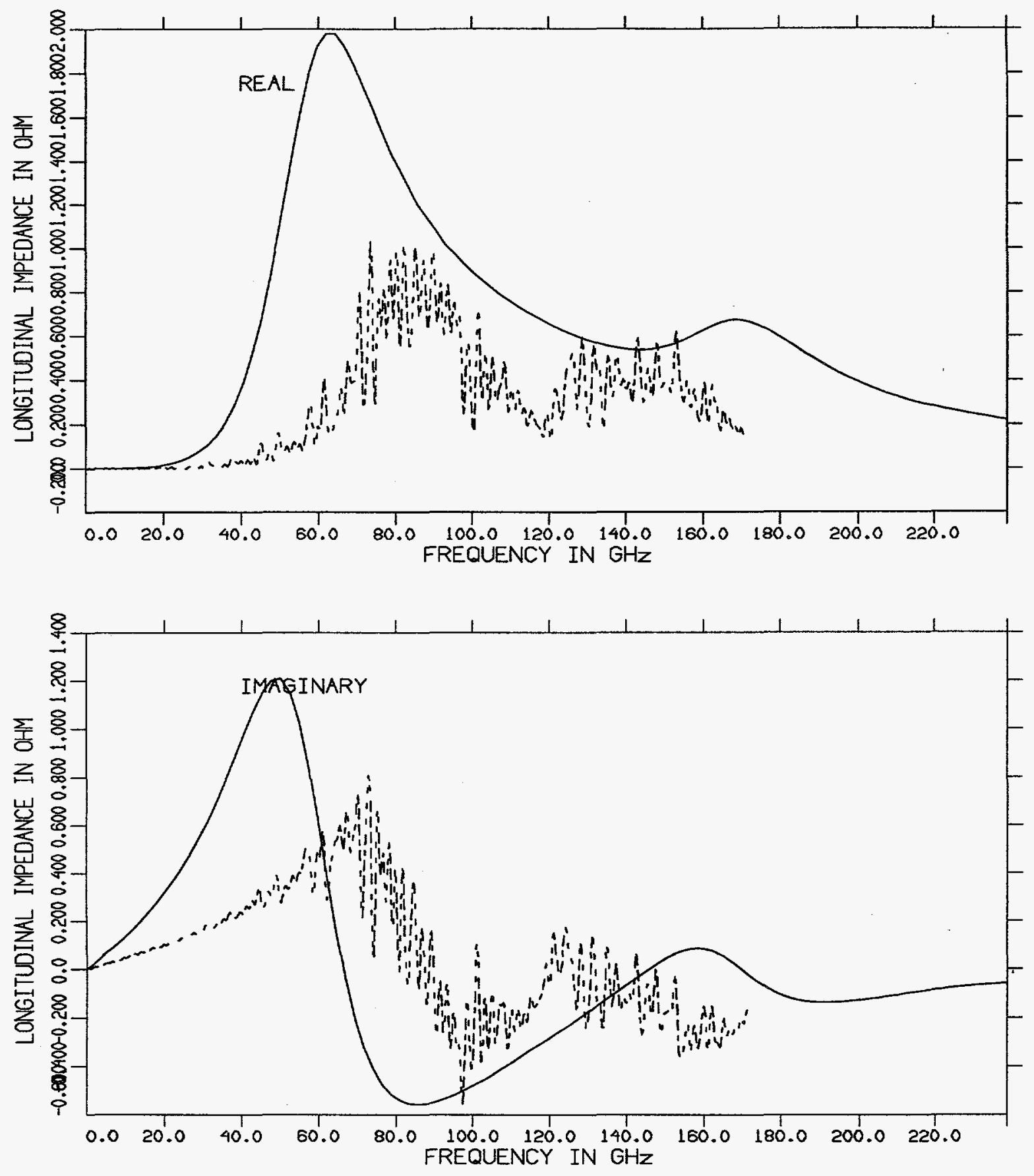

Figure 4.9: Coupling impedance of the hole with radius $1 \mathrm{~mm}$. Solid line from the variational calculation and dashed line from MAFIA-T3 simulation. 

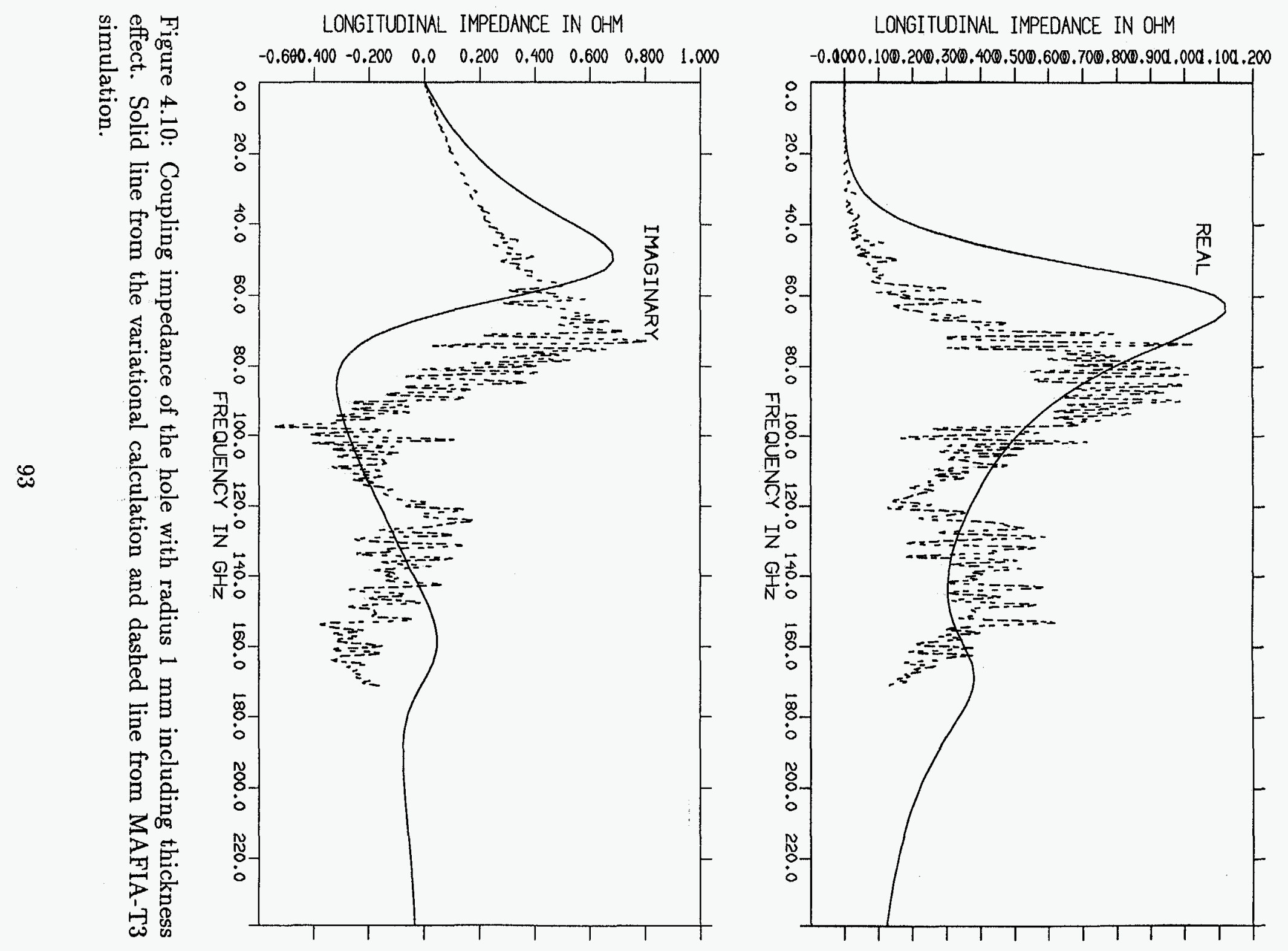



Figure 4.11: Coupling impedance of the hole with radius $2 \mathrm{~mm}$. Solid line from the variational calculation and dashed line from MAFIA-T3 simulation. 

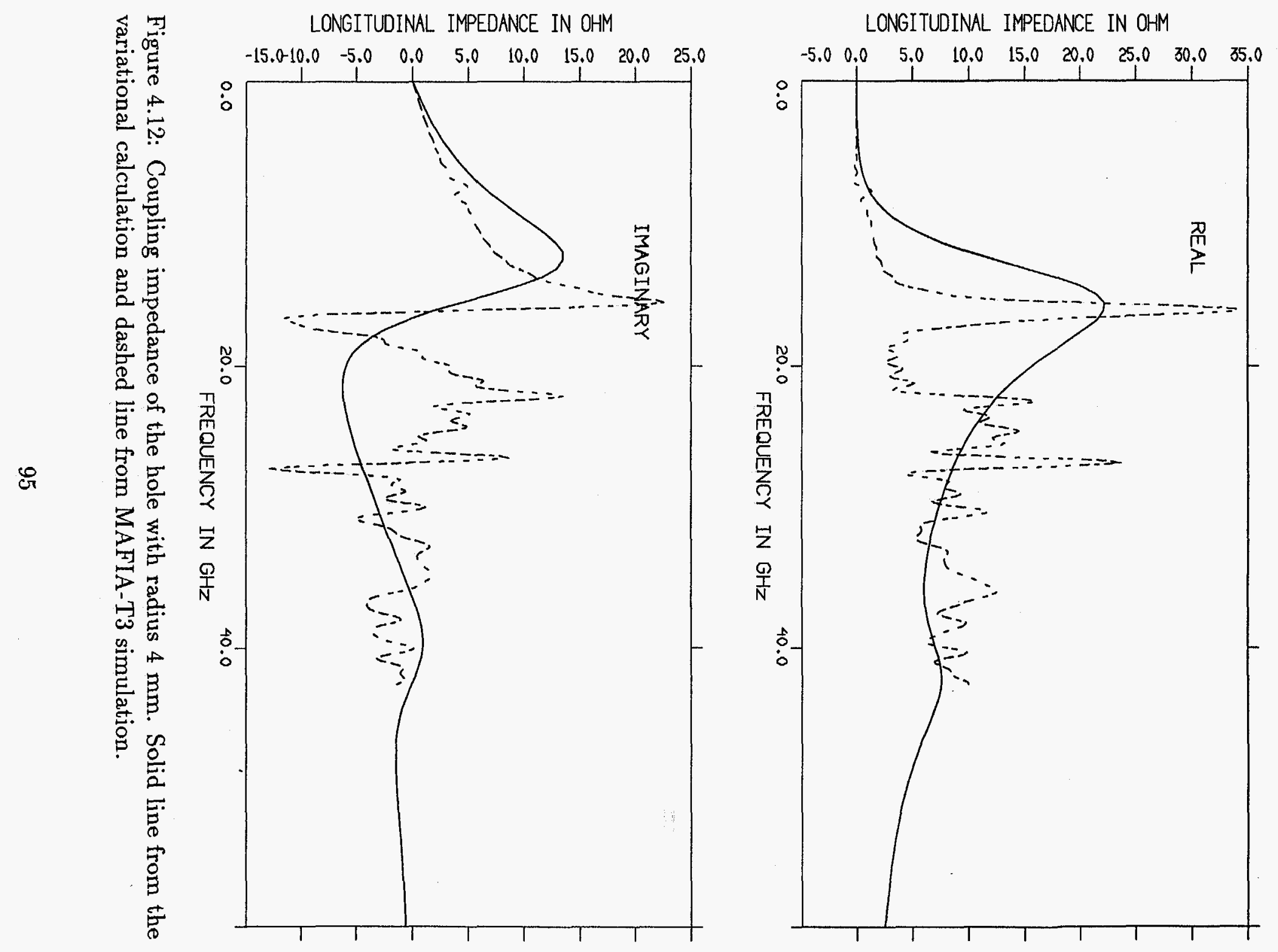


\section{Chapter 5}

\section{Summary and Conclusion}

In this work, we have derived analytic formulas using a variational method for the coupling impedance of a hole in an infinite screen and in a rectangular waveguide. The waveguide is considered as a model geometry resembling a real accelerator beam pipe. Utilizing Bethe's solution for the tangential electric field as our assumed field in the hole, we have shown that the coupling impedance can be expressed as a sum of finite functional series. The functions appearing in the formula are all well tabulated [1], enabling us to evaluate the numerical values readily.

When we compare our variational formula for the impedance with a well-known low frequency formula, we find that the two results agree.

Finally we have compared our variational results with the impedance obtained by the Fourier transform of the wake potential data from the program MAFIA-T3. We have shown a good qualitative agreement between two different methods in a wide frequency range.

Although one can in principle use MAFIA-T3 to calculate the impedance for a realistic accelerator beam pipe with holes, it will be very difficult to interpret or to understand the numerical data. In particular, when we try to parameterize the 
impedance with a model, for example the broadband resonator model, we may not know whether the exact shape of the beam pipe is important or not. If important, we must include a beam pipe dimension as a parameter in order to fit the numerical data. But we simply cannot answer such questions from numerical results alone.

From the analytical treatment presented in this work, we have shown that important parameters such as the resonant frequency and the bandwidth of resonance peak can be derived as functions of the dimensionless quantity $k d$, where $k$ is the free-space wave number and $d$ is the radius of a hole. Since these two parameters together with the slope of impedance at low frequency are enough to describe a resonator, our findings are very useful in understanding a complicated behavior of impedance obtained by numerical programs.

In conclusion, we have raised the treatment of the hole-coupling problem from the analysis valid in the low frequency range to one valid in a wide range of frequencies. By doing so we have succeeded in enlarging our understanding of the hole-coupling problem.

As a further investigation, we may continue to develop a semi-analytical method for the estimate of the coupling impedance of an arbitrarily shaped aperture in a realistic beam pipe. This effort may have dual purposes. First, it can be used to verify the purely analytical result, especially the one from an approximate method such as a variational method which we considered in this work. Secondly, it may enable us to calculate the impedance without a severe requirement on computer time and memory which often limits the usefulness of the purely numerical method such as the computer code MAFIA-T3. 


\section{Bibliography}

[1] Abramowitz, M. and I. A. Setgun, Handbook of Mathematical Functions With Formulas, Graphs, and Mathematical Tables, National Bureau of Standards Applied Mathematics Series 55, 1972.

[2] Baker, B. B. and E. T. Copson, The Mathematical Theory of Huygens' Principle, 2nd Ed., Oxford University Press, 1950.

[3] Bouwkamp, C. J., Philips Research Reports No. 5, 401-442, 1950.

[4] Bouwkamp, C. J., "Diffraction Theory," Reports on Progress in Physics, Vol. XVII, 35, 1954.

[5] Bethe, H. A., "Theory of diffraction by small holes," Phys. Rev., Vol. 66, 163, 1944.

[6] Bladel, J. van, Electromagnetic Fields, Reprinted by Hemisphere Publishing Corp., New York, 1985.

[7] Chao, A. W., Physics of Collective Beam Instabilities in High Energy Accelerators, John Wiley \& Sons, Inc., New York, 1993.

[8] Chen, H. C., Theory of Electromagnetic Waves, A Coordinate-Free Approach, McGraw-Hill Book Company, Inc., New York, 1983. 
[9] Collin, R. E., Field Theory of Guided waves, 2nd Ed., IEEE Press, New York, 1991.

[10] Gluckstern, R. L., F. Neri, "Longitudinal Coupling Impedance for a Beam Pipe with Cavity," IEEE Trans. Nuclear Sci., Vol. NS-32, No. 5, 2403, 1985.

[11] Gluckstern, R. L., " Coupling Impedance of a Single Hole in a Thick Wall Beam Pipe," CERN SL/AP 92-05, 1992.

[12] Gluckstern, R. L. and J. van Zeijts, "Coupling Impedance of a Hole in an Elliptical Beam Pipe,” CERN SL/AP 92-18, 1992.

[13] Gradshteyn, I. S. and I. M. Ryzhik, Table of Integrals, Series, and Products, Corrected and Enlarged Edition, Academic Press, Inc., New York, 1965.

[14] Harrington, R. F., Time Harmonic Electromagnetic Fields, McGraw-Hill Book Company, Inc., New York, 1961.

[15] Huang, C., R. D. Kodis and H. Levine, "Diffraction by apertures," J. Appl. Phys., Vol.26, 151, 1955.

[16] Jackson, J. D., Classical Electrodynamics, 2nd Ed., John Wiley \& Sons, Inc., New York, 1975.

[17] Klatt, R. and et al., "MAFIA - A Three Dimensional Electromagnetic CAD System for Magnets, RF Structures, and Transient Wakefield Calculations," Proceedings of the 1986 Linear Accelerator Conferences, 276, 1986.

[18] Kurennoy, S. S., "On the Coupling Impedance of a Hole or Slot," CERN SL/91$29,1991$. 
[19] Levine, H. and J. Schwinger, "On the theory of diffraction by an aperture in an infinite plane screen. Part 1," Phys. Rev., Vol. 74, 958, 1948.

[20] Levine, H. and J. Schwinger, "On the theory of diffraction by an aperture in an infinite plane screen. Part 2," Phys. Rev., Vol. 75, 1423, 1949.

[21] Levine, H. and J. Schwinger, "On the theory of electromagnetic wave diffraction by an aperture in an infinite plane conducting screen," Commun. Pure Appl. Math., Vol. 3, 355, 1950.

[22] Mentzer, J. R., Scattering and Diffraction of Radio Waves, Pergamon Press, New York, 1955.

[23] Rumsey, V. H., "Reaction concept in electromagnetic theory," Phys. Rev., Vol. 94, 1483, 1954.

[24] Schelkunoff, S. A., "Some equivalence theorems of electromagnetic and their application to radiation problems," Bell Syst. Tech. J., Vol. 15, 92, 1936.

[25] Schelkunoff, S. A., "On diffraction and radiation of electromagnetic waves," Phys. Rev., Vol. 56, 308, 1939.

[26] Schelkunoff, S. A., "Kirchhoff's formula, its vector analogue and other field equivalence theorems," Comm. Pure Appl. Math., Vol. 4, 43, 1951.

[27] Stratton, J. A. and L. J. Chu, "Diffraction theory of electromagnetic waves," Phys. Rev, Vol. 56, 99, 1939.

[28] Stratton, J. A., Electromagnetic Theory, McGraw-Hill Book Company, Inc., New York, 1941. 
[29] Tai, C.-T., Dyadic Green's Functions in Electromagnetic Theory, Intext Educational Publisher, Scranton, 1971.

[30] Watson, G. N., A Treatise on the Theory of Bessel Functions, Cambridge University Press, 1958.

[31] Weiland, T., "Transverse Beam Cavity Interaction. Part I: Short range forces," NIM, Vol. 212, 13, 1983. 